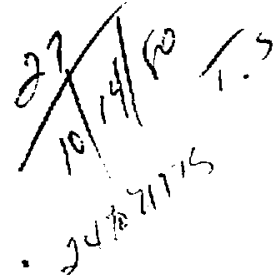

\title{
An experimental study of finite larmor radius effects
}

Kenneth William Struve

(Ph.D. Thesis)

August 1980 


\section{An Experinental Study of Finite Larmor Radius Effects In a Linear Z-PInch}

\section{By}

KENNETH WIL: :AM STRUVE

B.S. (Brigham Young University) 1971

M.S. (Brighan Young University) 1973

\section{DESERTATION}

Sabuitted in partial satisfaction of the requireneats for the degree of

\section{DOCTOR OF PBILOSOPHY}

in

\section{Engineering-Applled Sclence}

in the

\section{GRADUATE DIVSION}

of the

\section{UNIVERSITY OF CALIFORNLA}

DAVIS

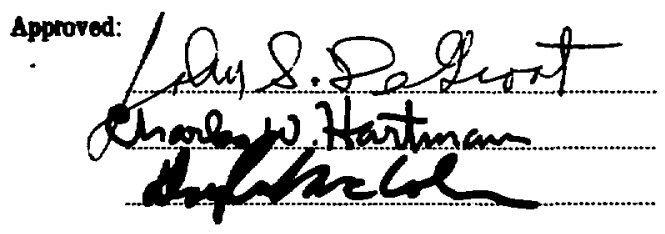

Connittee in Charge

Deposited in the University Library 
Kenneth W. Struve

Augus +1980

Applied Science

An Experimental Study of Finite Larmor Radius Effects

in a Lineor Z-pinch

\section{ABSTRACT}

Linear Z-pinches in $A r, K r, X e, N_{2}$, and He are experimentally studied in regimes where strong finite Larmor radius effects could provide a significant stabilizing effect. Scaling arguments show that for deuterium such a pinch has an electron line density of arder $2 \times 10^{15} / \mathrm{cm}$. For higher $Z$ plasmas a higher line density is allowed, the exact value of which depends on the average ion charge. The pinch is formed by puffing gas axially through the cathode towards the anade of an evacuated pinch chamber. When the gas reaches the anode, the pinch bank is fired. The pinch current rises in 2 to $3 \mu$ sec to a maximum of 100 to $200 \mathrm{kA}$. The pinch bank capacitance is $900 \mu \mathrm{F}$, and the external inductance is $100 \mathrm{nH}$. Additionally, the bank is fused to increase dIfdt. The primary diagnostics are a froming comera. a spatially resolved Mach-Zehnder interferometer, and $X$-ray absorption. It is found thet the initially conically-shaped gas jet pinches to axis in a time proportional to $f \propto \rho_{0}^{-1 / 2}$, rather than the $\rho_{0}^{-1 / 4}$ scaling typical of the snowplow pinch. Observed linear pinch instabilities 
hove growth rates more than a factor of ten less than the MHD model. Instability types are found to scale with the ratio of the finite Larmor radius divided by the pinch radius, which requires determining the averoge ion charge for higher $Z$ plasmas. The average ion charge is calculated by an approximate method for Ar plasmas. Least squares fits of Abel inverted electron densities show that the plasma has a Bennett density profile during the peak compression. It is found that FLP effects are important for high $Z$, low $T$ pinches. For low $Z$ pinches microinsfabilities dominate and prevent enhanced confinement at low line densities. An onode voltage spike and associated X-ray burst is observed to coincide with a sausage-like instability. X-ray energies are measured to be of order $e$ times the anode valtage. It is concluded that these instabilities are generated by localized, anomalously resistive regions, causing enhanced healing and subsequent rapid pinch expansion. 


\section{Acknowledgments}

Many people have assisted in the completion of this project. I am especially grateful to Charles Hortman who conceived the experiment and provided valuable guidance and support. Appreciation is extended to Dick Munger who was instrumental in the initial sef up of the experiment. Special thanks also to the fallowing who provided technical skills and guidance: John Clark for advice and help with the laser optics, Bob Canaan for help with the TV system, which was graciously loaned by the electron beam group of M-division, Doyle Rogers and his crew for advice and help with electronics problems, Bob Riley and his crew for mechanical guidance, Dave Applegate and Orrin Kommerer who helped run the experiment during its later stages. I'd also like to thank the M-division administrators, especially Mel Harrison, for support and encouragement. Finally, I am greatly apprecialive to my wife Susan, for her patience and encouragement during these past few years, and for her help in typing the hand-written text into the computer.

It should olso be mentioned that this thesis was processed by the TRIX AC / RED dialect on the Octopus and MFE computer networks at LLNL. 


\section{Table of Contents}

CHAPTER I Introduction $\ldots \ldots \ldots \ldots \ldots \ldots \ldots \ldots \ldots \ldots \ldots \ldots \ldots \ldots \ldots \ldots \ldots$

1.1. Motivation $\ldots \ldots \ldots \ldots \ldots \ldots \ldots \ldots \ldots \ldots \ldots \ldots \ldots \ldots \ldots \ldots$

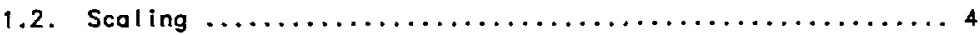

1.2.1. Finite Larmor radius scalling $\ldots \ldots \ldots \ldots \ldots \ldots \ldots \ldots$

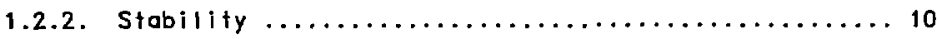

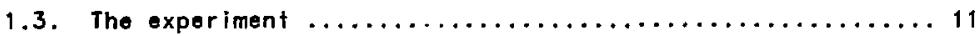

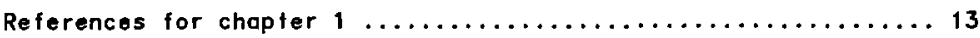

CHAPTER 2 Description of the Experiment $\ldots \ldots \ldots \ldots \ldots \ldots \ldots \ldots \ldots$

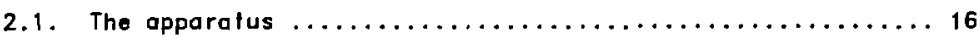

2.1.1. The capacitor bank and pinch circuitry $\ldots \ldots \ldots \ldots, 16$

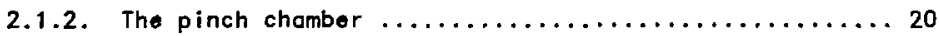

2.1.3. Pulsed gas valves $\ldots \ldots \ldots \ldots \ldots \ldots \ldots \ldots \ldots \ldots \ldots \ldots \ldots \ldots \ldots \ldots \ldots, 22$

2.1.4. The plasma gun $\ldots \ldots \ldots \ldots \ldots \ldots \ldots \ldots \ldots \ldots \ldots \ldots \ldots \ldots \ldots \ldots \ldots, 24$

2.1.5. Timing $\ldots \ldots \ldots \ldots \ldots \ldots \ldots \ldots \ldots \ldots \ldots \ldots \ldots \ldots \ldots \ldots \ldots \ldots \ldots \ldots \ldots, 27$

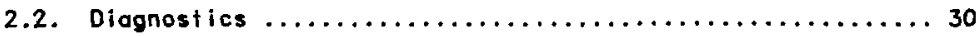

2.2.1. Framing cameras $\ldots \ldots \ldots \ldots \ldots \ldots \ldots \ldots \ldots \ldots \ldots, 30$ 
2.2.2. Loser interferometers $\ldots \ldots \ldots \ldots \ldots \ldots \ldots \ldots \ldots \ldots \ldots$

2.2.3. $x$-ray measuraments $\ldots \ldots \ldots \ldots \ldots \ldots \ldots \ldots \ldots \ldots \ldots \ldots$

2.2.4. Other measurements $\ldots \ldots \ldots \ldots \ldots \ldots \ldots \ldots \ldots \ldots \ldots$

References for chapter $2 \ldots \ldots \ldots \ldots \ldots \ldots \ldots \ldots \ldots \ldots \ldots \ldots$

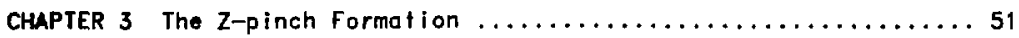

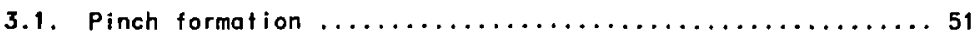

3.1.1. Gas flow into the chamber $\ldots \ldots \ldots \ldots \ldots \ldots \ldots \ldots \ldots$

3.1.2. Gas breakdown $\ldots \ldots \ldots \ldots \ldots \ldots \ldots \ldots \ldots \ldots \ldots \ldots \ldots \ldots$

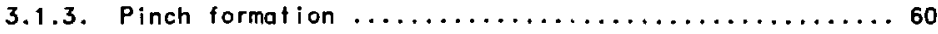

3.2. Examples of pinch formation and $1 \mathrm{ifetimes} \ldots \ldots \ldots \ldots \ldots \ldots 71$

3.2.1. The gas-puff initiated $z-p i n c h \ldots \ldots \ldots \ldots \ldots \ldots$

3.2.2. The plasma gun initiated $z-p i n c h \ldots \ldots \ldots \ldots \ldots$

3.3. Deduced parameters $\ldots \ldots \ldots \ldots \ldots \ldots \ldots \ldots \ldots \ldots \ldots \ldots \ldots \ldots \ldots$

3.4. Eloctron density profilos $\ldots \ldots \ldots \ldots \ldots \ldots \ldots \ldots \ldots \ldots$

Reforences for chapter $3 \ldots \ldots \ldots \ldots \ldots \ldots \ldots \ldots \ldots \ldots$

CHAPTER 4 The Pinch Break-up and Observed Instabilitios .......991

4.1. Description of the break-up processes $\ldots \ldots \ldots \ldots \ldots \ldots \ldots 92$

4.1.1. Scenario $\ldots \ldots \ldots \ldots \ldots \ldots \ldots \ldots \ldots \ldots \ldots \ldots \ldots \ldots \ldots$

4.1.2. Types of pinch instabilities ................ 95

4.1.3. Other features $\ldots \ldots \ldots \ldots \ldots \ldots \ldots \ldots \ldots \ldots \ldots \ldots$

4.2. Meosured parameters $\ldots \ldots \ldots \ldots \ldots \ldots \ldots \ldots \ldots \ldots \ldots \ldots \ldots$ 
4.2.1. X-roy absorption moosuraments ............. 102

4.2.2. Growth rate and fluid volocity moasuraments ....... 104

4.2.3. Instablility type scaling with the FLR parameter .... 106

References for chapter $4 \ldots \ldots \ldots \ldots \ldots \ldots \ldots \ldots \ldots \ldots \ldots \ldots$

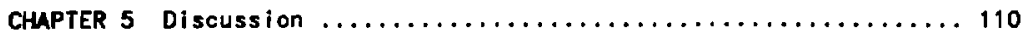

5.1. Linear growth rate comparisons $\ldots \ldots \ldots \ldots \ldots \ldots \ldots \ldots \ldots 111$

5.1.1. Analytical techniques $\ldots \ldots \ldots \ldots \ldots \ldots \ldots \ldots \ldots \ldots \ldots 112$

5.1.2. Skin current model growth rates $\ldots \ldots \ldots \ldots \ldots \ldots \ldots 117$

5.1.3. Diffuse current model growth rates $\ldots \ldots \ldots \ldots \ldots \ldots 118$

5.1.4. Finite Larmor radius effect on the growth rates .... 123

5.1.5. Comparisons of observed with calculated growth rates 128

5.2. The pinching dynamics and non $M H D$ effects $\ldots \ldots \ldots \ldots \ldots 129$

5.2.1. Pinching dynamics $\ldots \ldots \ldots \ldots \ldots \ldots \ldots \ldots \ldots \ldots \ldots \ldots$

5.2.2. The voltage spike and the sausage-like disruption ... 137

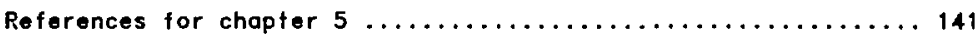

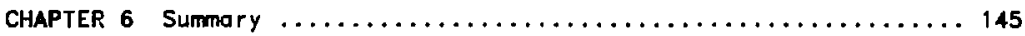

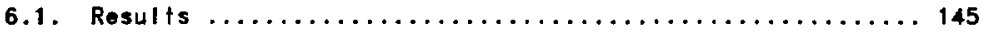

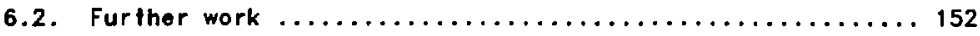

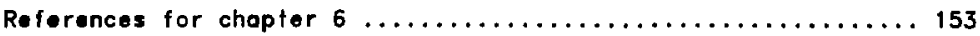


APPENDIX A Pulsed Gas Valve and Gas Flow Dynamics ........... 154

A.1. Construction and operation of the gas valve $\ldots \ldots \ldots \ldots \ldots 154$

A.2. Gos dynomics $\ldots \ldots \ldots \ldots \ldots \ldots \ldots \ldots \ldots \ldots \ldots \ldots \ldots \ldots \ldots \ldots$

References for appendix A .................... 167

APPENDIX B Laser Interferometry and Abel Inversion ........... 169

B.1. General considerations $\ldots \ldots \ldots \ldots \ldots \ldots \ldots \ldots \ldots \ldots \ldots \ldots$

B.2. Abel inversion $\ldots \ldots \ldots \ldots \ldots \ldots \ldots \ldots \ldots \ldots \ldots \ldots \ldots \ldots \ldots \ldots$

References for appendix B ..................... 179

APPENDIX $C$ Determination of the Effective Ion Charge ......... 180

References for appendix $C \ldots \ldots \ldots \ldots \ldots \ldots \ldots \ldots \ldots \ldots$

APPENDIX D Electron Density Profiles .................. 189 
CHAPTER 1

Introduction

1.1. Motivation

The earliest efforts in controlled thermonuclear fusion were with the linear z-pinch. (By canvention, Z-pinch denotes the magnelic configuration in which a sirong current flows in the $z$ direction in a plasma inducing a magnetic field in the $\theta$ direction. Here $z$ and $\theta$ refer to coordinates in cylindrical geometry. The self-induced magnetic field then squeezes, or pinches the plasma, thus confining it.) But these earlier experiments showed that the pinch was unstable to sausage and $k$ ink modes. In the sausage mode the plasma is similar 
in shape to link sausage in that there are several islands of plasma linked by thin filaments. In the kink mode there is a gross motion of the plasma column oft the axis of symmetry. Tinis can be helical in structure and look much like a spring. The pinch instability was later predicted with a fluid theory using the energy principle by Kruskal and Schwarzschild, 1 and still later including a distributed current by Newcomb. ${ }^{2}$ A related technique which also uses the fluid theory is to perturb the equilibrium fluid equations and solve for the instability growth rates. Kadomtsev ${ }^{3}$ summarizes this approach using a skin current madel showing that a wavelength can always be found such that one of the modes is unstable. The energy principle shows that this is nat quite as serious with distributed currents, and that with density gradients sufficiently small only the lowest order kink mode is unstable.4 Although more complicated, Z-pinch growth rate calculations using a diffuse current model have also been done by several researchers.5.6 They generally find that rie growth rates with an axially stabilizing field ore sensitive to the density profiles.

As a result of the theory, much effort has gone into altering the magnetic field configuration to stabilize the plasma. This usually involves adding an externally produced magnetic field roughly equal to, or greater in magnitude to the self-induced fields. Some examples are the lokamak and the stabilized pinch. The goal of the various epproaches is to confine the plasma sufficiently long, and with, a high enough particle density, that the fusion energy released exceeds the 
expressed as the Lawson criterion, which requires the producl of the particle density and cunfinement time nt be $\geq 10^{14}$ sec $/ \mathrm{cm}^{3}$ for deuterium-tritium reactions and with a temperature $\geq 10 \mathrm{keV}$. The density in these configurations is limited by the maximum external magnatic field which can be produced to roughly $10^{14} / \mathrm{cm}^{3}$. Therefora the confinement time must be nearly one second.

However, if an externally produced magnetic field is not used, one could consider a pinch with much higher magnetic fields and particle densities, and proportionally shorter confinement times. If the densities were made sufficiently large, then the required confinement time could be decreased to be less than the the time for instabilities to develop. The fluid theory predicts instability growth times to be roughly equal to the ratio of the pinch radius to the ion sound velocity. This requires, however, densities of the order of solid densities and correspondingly large magnetic fields, 7 Experimentally the observed growth times are found to be 10 to 100 times longer than those predicted by the fluid theory. ${ }^{8}$ This relaxes the density requirements somewhat, and allows the concept of a Z-pinch reactor to become feasible.

One mechanism that has been shown to have stabilizing effects with other configurations ${ }^{9}$ is the consideration of finite Lormor radius offects (hereafter denoted FLR). Hartman ${ }^{10}$ has proposed that these offects could be a stabilizing mechanism for the linear z-pinch, provided the pinch is operated in regimes where these effects are 
strong. In the fluid theory it is assumed that each ion Larmor radius is small compored to the dimensions of the plasma. When the ion Larmor radius becomes large the fluid theory breaks down. For nearly-fluid plasmas, these offects can bo treatod by adding viscous terms to the pressure tensor. 11 However, in regimes where these effects would be strong, the corrections also break down and need to be replaced with a porticle theory.

Presentiy there exists no theory nor satisfactory numerical simulation which includes strong finite orbit effects in the linear Z-pinch. Nielsen, ef a ${ }^{12}$ hove done three-dimensional particle simulations of the linear Z-pinch, but cannot investigate the high density regimes because of the need to follow many more particles than is possible with their code. Because of the complexity of the problem, the lack of a satisfactory theory, and the possibility of a significant stabilizing effect, it was decided to investigate experimentally these regimes.

\subsection{Scaling}

\subsubsection{Finite Larmor radius scaling}

Following the arguments of $\operatorname{Hartman}^{13}$, consider a Z-pinch where finite orbit effects would be important. This is a pinch which would 
be, at most, only several ion orbits in diameter. For diffuse pinches the Larmor radius is a function of radius.

$$
a_{i}=\frac{v_{t}}{\omega_{c i}}=\frac{2 \pi r\left(m_{i} k T_{i}\right)^{1 / 2}}{Z_{a v} \propto \mu_{0} I(r)}
$$

where $Z_{a r}$ is the average ion charge, and $I(r)$ is the integral of the current Jensity.

$$
I(r)=\int_{0}^{r} J_{z}\left(r^{\prime}\right) 2 \pi r^{\prime} d r^{\prime}
$$

Note that MKS units are used, as is the convention throughout the poper. However, when numbers are required, these will be expressed in cgs units, since these are commonly used units in experimental plasma physics.

With a uniform current density, the Larmor radius is proportional to $1 / r$. Clearly, the Larmor radius becomes larger than the pinch radius as $r$ opproaches zero. To avoid this difficulty, find the overage Larmor radius by integrating over the particle distribution. 


$$
\left\langle a_{i}\right\rangle=\frac{\int_{0}^{\infty} a_{i}(r) n(r) 2 \pi r d r}{\int_{0}^{\infty} n(r) 2 \pi r d r}
$$

The number of ion orbits in the diameter is then the ratio of the pinch radius a to the average Larmor radius $\left\langle a_{p}\right\rangle$. Since this number is dependent on the current and densify profiles, consider the results of three special cases os examples. Defining $s$ as,

$$
s=\frac{\mu_{0} I_{0}}{2 \pi\left(m_{i} k T_{i}\right)^{I / 2}}
$$

Case 1. Uniform current and electron densitias

$$
\frac{a}{\left\langle a_{i}\right\rangle}=\frac{s}{2}
$$


Case 2. Uniform current density and $n_{\bullet}(r)=n_{0}(0)\left[1-(r / a)^{2}\right]$

$$
\frac{a}{\left\langle\sigma_{i}\right\rangle}=\frac{3}{8} s
$$

Cose 3. Bennett profiles't in current and electron densitios.

$$
\frac{a}{\left\langle\sigma_{i}\right\rangle}=s \frac{b^{1 / 2} a}{\pi}
$$

A Bennett density profile is given by

$$
n(r)=\frac{n(0)}{\left(1+b r^{2}\right)^{2}}
$$

The parameter $b$ describes the width of the Bennett profile, namely. $n\left(b^{-1 / 2}\right)=n(0) / 4$. This profile arises from considering the electron drift velocity and the plasma temperature independent of $r$. If $b^{-l / 2}$ is defined as the plesmo radius $a$, then the number of ion. orbits is $s / \pi$, which is very nearly the resulf for cose 2 . It is determined by

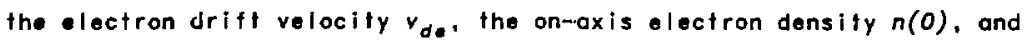
the temperafure, and equals 


$$
b=\frac{\mu_{0}}{8} e^{2} v_{d \theta}^{2} \frac{n(0)}{k\left(T_{0}+T_{i}\right)} .
$$

The quantity $s$ detined in eq. (1.4) can be shown to be the ratio of the pinch radius to the ion Larmor radius at the outer edge of the pinch. This is done by replocing $r$ with $a$ in eq. (1.1). Since this has appeared in the three examples given, it seems sensible to consider $s$ as a scaling parameter relating to the number of ion orbits acrass the width of the pinch.

The temperature dependence of eq. (1.4) can be eliminated by considering a balance between the mognetic field and particle pressure. For arbitrary $Z$ and $T$ eq. (1.4) becomes.

$$
s=\left[Z_{o v}\left(1+\frac{T_{0}}{T_{i}} Z_{a v}\right) \frac{2 \theta^{2} \mu_{o}}{\pi m_{i}} \pi o^{2} n_{0}\right]^{1 / 2} .
$$

In cgs units, with $T_{i}=T_{j}$, the result for deuterium is,

$$
s_{D}=\left(\frac{\pi n . a^{2}}{1.6 \times 10^{15}}\right)^{1 / 2}
$$

The subscript implies that the expression is evaluated for deuterium. For higher $z$ ions, and with unequal temperatures, $s_{0}$ is madified by, 


$$
s=\left[\frac{Z_{o v}\left(1+Z_{o v} \frac{T_{i}}{T_{i}}\right)}{\frac{m_{i}}{m_{D}}}\right]^{1 / 2} s_{D} .
$$

The quantity $\pi n, a^{2}$ in eqs. $(1.10)$ and $(1.11)$ is the number of eloctrons per unit length, and is called the electron line density. Thus the number of ion orbits in a hydrogen or deuterium pinch scales only with the line density, and is independent of the ion temperature. For higher $z$ plasmas the temperature plays a more important role in that it determines the charge stote $Z_{a v}$. This complicates the analysis by requiring knowledge of both the temperature and electron density to determine the FLR scaling.

From these scaling arguments it can be seen that it is necessary to maintain a low line density to obtain a pinch where FLR effects are important. If the current and electron density profiles from case 3 are used, the scaling parameter $s$ must equal approximately $\pi$ to have a pinch containing one ion orbit. For deuterium, this dictates an electron line density of $1.6 \times 10^{16}$ per $\mathrm{cm}$. For higher $Z$ plasmas this density scales approximately as $z^{1 / 2}$, if fully ionized. 


\subsubsection{Stability}

If, however, the line density is made too small, the electron drift velocity becomes large enough to exceed the threshold for the lower hybrid drift instability. ${ }^{15}$ Hartman 16 has shown that when this happens there is a localized, rapid heating, and a subsequent expansion of the pinch column. That this threshold also scales with the line density and the FLR parameter $s$ is shown by considering the ratio of the electron drift velocity $v_{\text {do }}$ to the ion thermal velocity $v_{f}$, and by using the same pressure balance condition used to derive eq. (1.10).

$$
\begin{aligned}
\frac{v_{d e}}{v_{i j}} & =\frac{J_{z}}{n_{\theta} e}\left(\frac{m_{i}}{k T_{i}}\right)^{1 / 2} \\
& =\frac{4 Z_{a v}^{1 / 2}\left(\frac{1}{Z_{a v}}+\frac{T_{0}}{T_{i}}\right)}{s}
\end{aligned}
$$

Combining eqs. (1.12) and (1.13) gives o result related to the FLR parameter for deuterium.

$$
\frac{\frac{4}{Z_{a v} / 2}\left(\frac{1}{Z_{a v}}+\frac{T_{0}}{T_{i}}\right)^{1 / 2}\left(2 \frac{m_{D}}{m_{i}}\right)^{1 / 2}}{s_{D}}
$$


To avoid this instability it is necessary to require that this ratio of velocities be $\leq 1$. For deuterium plasmas, for example, $s_{D}$ must be $\geq 8$. For an argon plasma, this requirement is not as stringent. The parameter $s$ in this case must be only $\geq 0.3$.

These results suggest the possible existence of a stability window with a line density low enough to obtain strong FLR effects and yet still high enough to avoid the lower hybrid drift instability. Pinches formed with line densities below the lower limit would then exhibit very large microinstability growth rates, and those formed with line densities well above those needed for FLR considerations would exhibit the well-known MHD type instabilities and growth rates. Presumably, those formed with line densities within this window would be stable, or at least exhibit growth rates much slower than predicted by the MHD theory.

\subsection{The Experiment}

Considering the number of Z-pinch experiments which have been done previously, one would expect that FLR offects might have been observed. This is not the cose, however, since earlier pinches operoted at much higher line densities. They were usually initiated by breakdown along an insulator at the chamber wall. The current formed in o thin shell at the wall, which then collapsed radially, accruing 
mass as it swept in. If the fill pressure of the chamber was lowered sufficiently that one might see FLR effects, it was impossible to induce electrical breakdown of the gas with the available electrode volfages. For these reasons, most of the previous work was done with line densifies which did not permit observation of FLR effects.

This problem can be overcome by producing a localized high density region to allow gas breakdown, and by also controlling the exient of the region to insure a low line density. Low line density, and high local density can be ochieved by puffing gos between the electrodes and along the axis of a previously evacuated pinch chamber. The line density is then controlled by the amount of gas puffed into the chamber. Another technique to achieve the same result is to direct a plasma stream from a plasma gun into the electrode gap. Both techniques have been Iried, and will be discussed.

The gools of the experiment have been to; (1) set-up and operate o pinch in regimes where FLR effects might be important, (2) observe the instability types and measure their growth rates, and (3) determine what effect the line density has on the nature of the instabilities. Additionally, measured growth rates are compared with those predicted by the diffuse-current NHD theory, and with those ostimoted by a diffuse-current MHD theory which includes FLR corrections.

In chapter 2 the description of the experiment and diagnastics is given. The experimentally observed formation of the pinch is discussed in chapter 3 . In chapter 4 the instability types are given and 
categorized, and in chapter 5 the results are discussed, along with their implications. The results are summarized in chapter 6.

$$
\text { References for chapter } 1
$$

${ }^{1} M$. Kruskal and M. Schwarzschild, Proc. Roy. Soc. (London) A223, 348-360 (1954).

2w. B. Newcomb, Ann. Phys. (NY) 10, 232-267 (1960).

${ }^{3}$ B. B. Kadomtsev, in Reviews of Plasma Physics, edited by M. A. Leontovich (Consultants Bureau, New York, 1966), Vol. 2 , Pp. 153-199.

${ }^{4}$ Ibid., p. 183 .

${ }^{5}$ K. Hain and R. LUst, Z. Naturforsch. 13a, 936 (1958).

6. P. Goedbloed and H. J. I. Hagebeuk, Phys. Fluids 15, 1090 (1972).

7J. G. Linhart, Nucl. Fusion 10, 211 (1970).

${ }^{8}$ C. W. Hortman, G. Corlson, M. Hoffman, R. Werner, and D. Y. Cheng, Nucl. Fusion 17, 909 (1977). 
9M. N. Rosenbluth, N. A. Krall and N. Rostoker, Nucl. Fusion Supp I. Pt. 1, 143 (1962).

${ }^{10} \mathrm{C}$. W. Hartman, Lawrence Livermore Laboratory Report UCID-17118 (1976).

11K. V. Roberts and J. B. Taylor, Phys. Rev. Letf. 8, 197 (1962).

12D. Nielsen, J. Green, and O. Buneman, Phys. Rev. Lett. 42, 1274 (1979).

${ }^{13}$ Hartman, UCID-17118, P. 2.

14N. A. Krall, and A. W. Trivelpiece, Principles of Plasma Physics, (NcGraw-Hill, New York, 1973), P. 103.

15P. C. Liewer, and N. A. Krall, Phys. Fluids 16, 1953(1973).

${ }^{16}$ Hartman, UCID-17118, PP. 4,5 . 


\section{CHAPTER 2}

\section{Description of the Experiment}

To construct an experiment where finite Larmor radius effects are important it is necessary to maintain a low electron line density $\pi n_{0} a^{2}$ and yet maintain the number density $n_{0}$ along the axis high enough that breakdown can be achieved. This is accomplished by using a pulsed gas valve to provide a localized puff of gas along the pinch axis. Thus, the local density is kept high, while the line density is kept low. In this chapter I will discuss how this is done experimentally, give the details of the experiment, and discuss the diagnostics. 


\subsection{The apparatus}

\subsubsection{The capacitor bank and pinch circuitry.}

The finite Larmor radius z-pinch experiment was designed to use the "green river" capacitor bank and control circuitry in building 431 at LLNL. This bank was constructed in the late $50^{\circ} \mathrm{s}$ and consists of $120,7.5 \mu \mathrm{f} / 20 \mathrm{kV}$ capacitors each connected to a common pad with ten RG-213 coaxial cables. The cables are about 10 meters long, are green in color, and appear to flow to the pad; hence the name "green river" bank. Each capacitor has its own fire and crowbar ignitron, all fired from a separate capacitor through o disfribution panel. The total inductance of the system is $100 \mathrm{nH}$, with the time to current maximum being about $15 \mu \mathrm{sec}$. Refer to the circuit diagram in fig. 2.1 .

In order to increase the rate of current rise through the pinch. the first few microseconds of the bank current is shorted through a fuse placed in parallel with the pinch electrodes. The bank current is thereby allowed to rise slowly through the fuse until it blows, after which the current flows only through the pinch chamber. Additionally, a spark gap was located between the fuse and pinch anodes to prevent current flow through the pinch while the fuse is conducting. 


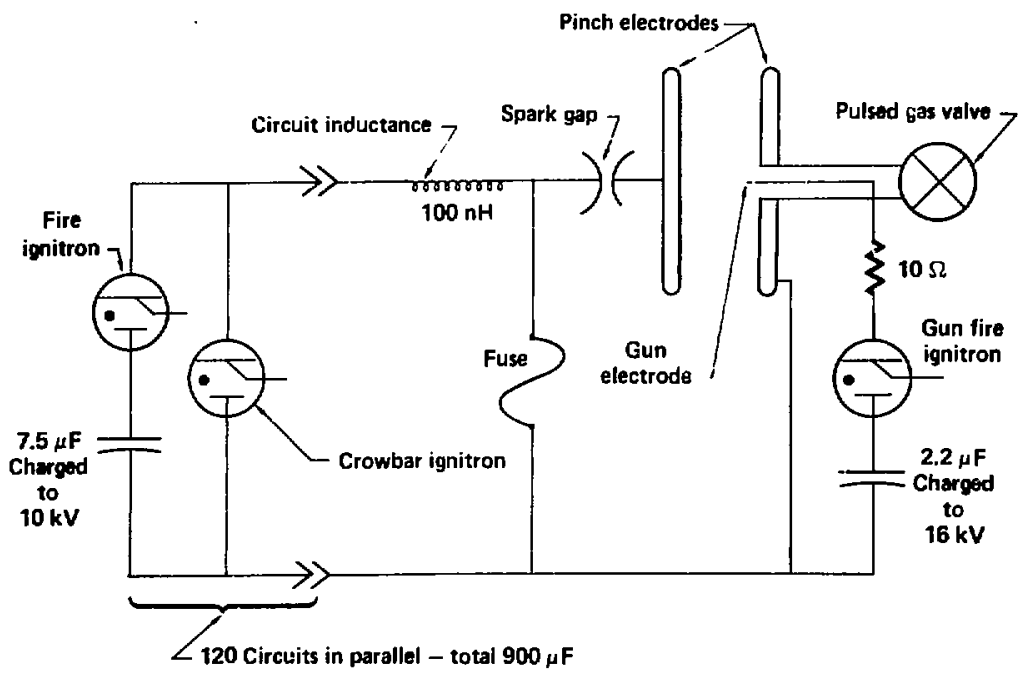

Figure 2.1. Finite Larmor radius Z-pinch circuit.

The gas pressure in the spark gap region is adiusted to hold off the voltage as current is flowing through the fuse. When the fuse blows there is a large, inductive voltage spike at the fuse anode which then breaks down the gap and allows current flow to the pinch anode. A spark plug was also placed in the spark gap chamber region to assist in breakdown of the gap. It is connected to the bank pad thraugh a $50 \Omega$ resistor. The spark gap separation ranged from 3.8 to $5.5 \mathrm{~mm}$. The seporation varied because the gaps were gradually worn down with each shot and hod to be replaced periodically. The gas fill is either $\mathrm{N}_{2}$ or 
operating pressures were found initially by hi-potting the spark gap to find the breakdown voltage as a function of pressure. Thereafter, the pressure was adjusted by trial and error: e.g., it is kept just low enough to breakdown the gap with the inductive voltage spike from the fuse.

The fuses consist simply of bare copper wires taped to the fuse terminals and imbedded in sand. See the diagram of the fuse terminals in fig. 2.3 for their location. Note that the assembly was extended below the pinch chamber to provide accessibility. The number and diameter of fuses varied for different bank charge voltages. Af a $4 \mathrm{kV}$ charge two 20 gauge wires were used. At other voltages, cambinations of wires were used to maintain the same ratio of the wire cross-sectional area to bank energy.

This technicue works very well and decreases the current rise time to 1 to $2 \mu \mathrm{sec}$. Fig. 2.2 shows the effect of the fuse operation on the pinch current rise time. The upper trace is the total current flowing from the pad. The lower frace is the pinch current only. Note the factor of ten increase in the rafe of current $r i s e$ in the pinch current. The disadvantages of the system were that the bucket holding the sand around the fuses had to be dropped with each shot and the fuses replaced, and that the sand was pulverized in the process. While changing the fuses and sand between shots, a fine dust rose and then settled on the experiment, making it difficult to keep the opfics and 


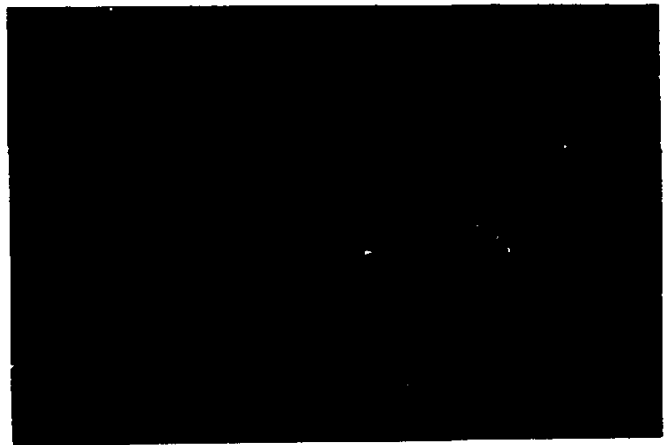

Figure 2.2. The effect of the fuse on the pinch current rise time. The upper trace is the total current and the lawer trace is the pinch current, bath $100 \mathrm{kA}$, div. and $5 \mathrm{\mu sec} / \mathrm{div}$. The difference between the two is the current through the fuse.

the operator clean. But, in spite of these problems, the fusing was very reliable and allowed use of a slower bank.

The remainder of the circuit is completed by the pinch fram the anode to the cathode. The plasma for the pinch comes from ionization of the gas which is puffed into the chamber through a hole in the cathode. Refer again to fig. 2.1. The electrode in the center of the gas flow channel serves either of two purposes: (1) If acts as a spork trigger to help form a conduction path, or (2) Without the $10 \Omega$ resistor shown in fig. 2.1 it becomes an electrode of a Cheng type plasmo gun.' The characteristics of the gun and gas valve are discussed in section 2.1.3. Some of the earlier shots were initiated 
with this plasma gun. However, most of the discussion will be of plasmas where the gun only acts as a breakdown trigger.

\subsubsection{The pinch chamber}

Two different vacuum chambers and a variety of electrode styles were used in the experiment. The first chamber was $57 \mathrm{~mm} 10 n g$. and allowed for a pinch length of $23 \mathrm{~mm}$. A second chamber was $210 \mathrm{~mm}$ long. and is the chamber with which most of the shots were done, and which is

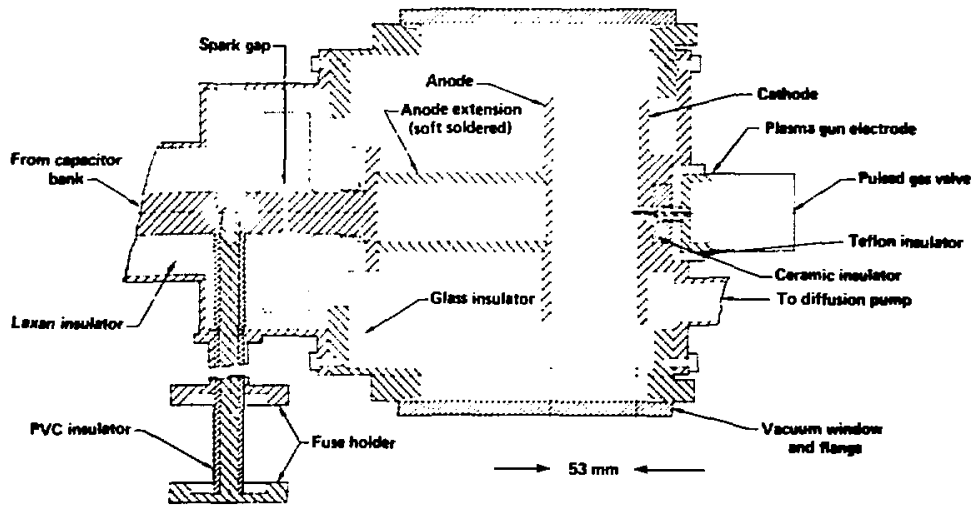

Figure 2.3. A cross-sectional view of the pinch chamber and fuse assembly. The long chamber with the adjustable length anode-cathode seporation is shown. The length is changed by replacing the anode extension with one of a different lengih. 
spark gap region remoined the same.

The pinch shamber wos constructed of brass as shown in fig. 2.3 . The anode and cathodes are of copper. An earlier version used tantalum electrodes. However. the tantalum which was platea on the vocuum windows during the shot was difficult to remove. The copper electrodes plated the windows equally fast, but they were more easily cleoned with solvents. The window plating problem wos also partially solved by using flange extensions to move the windows back about $30 \mathrm{~cm}$.

The glass insulator which was placed around the throat of the anode prevented the current from sharting to the chamber wall, which was the refurn conductor. At the other end, because of its larger diameter, the anode was only $2.9 \mathrm{~cm}$. from the wall. Breakdown did not occur however since the chamber wos held of o vacuum until the gas was puffed in through the opening in the cathode. The vacuum was typically in the $10^{-5}$ Torr range, with the chamber being pumped by a liquid nitrogen cooled o: I diffusion pump located roughly $4 \mathrm{~m}$ from the experiment. Typically, the capacitor bank was fired before the gas had time to fill the region befween the anode and chamber wall. Breakdown to the wall occurred only after the pinch collumn broke up, of which time it could not effect pinch characteristics. 


\subsubsection{Pulsed gas valves}

Two different pulsed gas valves were used. They were (1) a Berkeley puffatron valve, ${ }^{2}$ which was used on the initial sequence of shots, and (2) a fast gas valve modeled after the one used by Cheng ${ }^{3}$ with his deflagration gun. The puffatron was used because it was fast, it was available, being left over from a previous experiment, and because it is capable of puffing into a region known amounts of gas. The Cheng style fast gas valve was built and used with later shots to reduce the timing $i i t t e r$, and to increose the amount of gas puffed into the chamber.

The puffatron gas valve consists of a steel rod which is held on an 0-ring seal by springs, and a solenoidal coil which drives the rod off the seal when pulsed with a high current. The seal encloses a small plenum which had a volume of $.84 \mathrm{~cm}^{3}$ and had a stafic fill pressure that ranged anywhere from 100 Torr to several atmospheres. The gas valve rod assemb $1 y$ is $25 \mathrm{~mm}$ in diameter and $80 \mathrm{~cm}$ long, with the solenoid driving coil $100 \mathrm{~mm}$ in diameter, mounted at the end of the rod. The gas valve was mounted in the pinch cathode flange in a similiar position to the gos valve shown in fig. 2.3 .

The puffotron valve opens in $160 \mu \mathrm{sec}$ and has a jitter time of as much as $\pm 50 \mu s e c$. The current for the solenoid was supplied by a $2.2 \mu \mathrm{F}$ capacitor charged to $11 \mathrm{kV}$, which was fired and crow-barred with ignitrons. The current rise time was $15 \mu \mathrm{sec}$ and the peak current about $10 \mathrm{kA}$. 
The second puff valve, which was built and used with most of the shots, was modified from Cheng's design to allow use with the existing

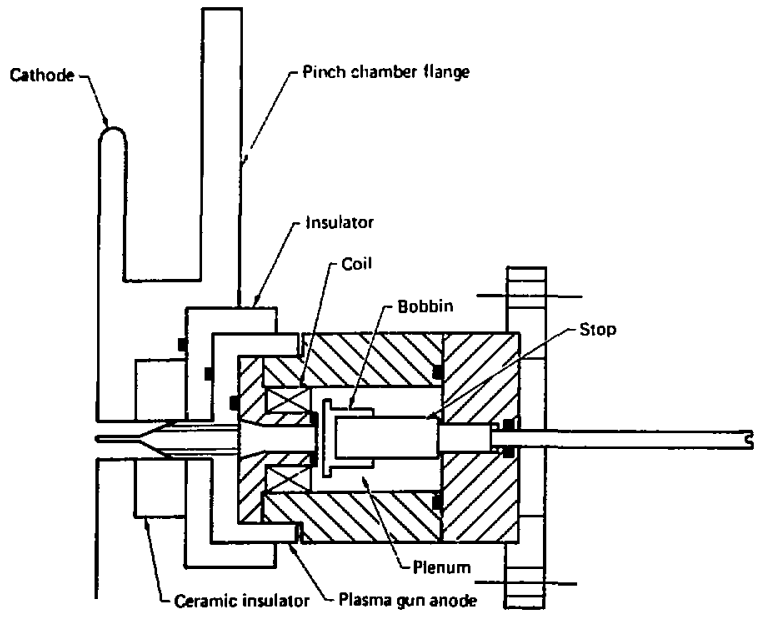

Figure 2.4. Cross-sectional view of the Z-pinch cathode showing the plasma gun and pulsed gas valve.

geometry in our Z-pinch electrode. Fig. 2.4 shows this gas valve and how it was positioned at the throat of the plasmo gun on the cathode flange. The seal is made by either a small copper disk or coil wound bobbin which is held against a rubber o-ring by ambient plenum pressure. Either the disk or the bobbin serves as a secondary coil of a transformer, and is shot off the seal as the primary coil is pulsed, thus opening the valve. When the disk reaches the stop, it rebounds with the same speed. Meosurements of the stop position show that the 
valve closes and does not reopen. A small spring was also inserted behind the disk to keep it on the seal while pumping the plenum. It was weak enough, however, to not affect the opening and closing times. A more detailed discussion of the construction and gas dynamics of the valve is given in appendix $A$.

The total quantity of gas entering the pinch chamber, as a function of time, is given in fig. 2.5. The derivation of these results is in appendix $A$. The vertical coordinate is the fractian of gas from the plenum which enters the chamber. The total number of particles is obtained by multipling by a constant, which is $3.3 \times 10^{20}$ for the Cheng style valve, and $1.5 \times 10^{19}$ for the Berkeley valve, and by the number of atmospheres of plenum fill pressure. The origin of the horizontal scale is the time the gas valve is fired. The valve opening time is measured to be $40 \mu$ sec. The delay in gos arrival is the sound transit time from the gas valve opening to the cathode plane.

\subsubsection{The plasmo gun}

As mentioned at the end of chapter 1, rather than puffing gas into the region between the electrodes, it is also possible to direct a plasma stream across the electrode gap to allowbreakdown but still maintain a low line density. The primary disadvantage of using the gun initiation was that because of the iitter in the gun fire time it was not possible to fuse the bank current to oblain shorter $r$ ise times in the pinch curiant. The gun used was a small version of Chengs 


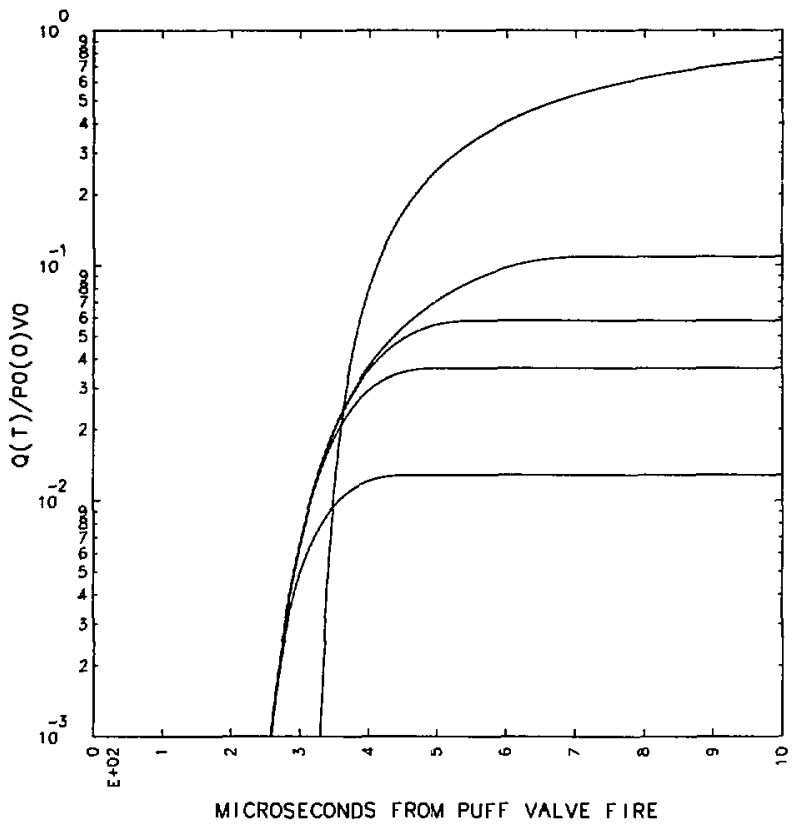

Figure 2.5. Total quantity of gas admitted to the pinch chamber from the fast gas valves as a function of time, as calculated from the gas dynamics. Each curve is a set of values for stop settings of $1 / 2,1,11 / 2$, and $21 / 2$ turns, at $.907 \mathrm{~mm} /$ turn. The highest curve is the output of the Berkeley puffatron valve.

deflagration gun. 4 The gun is of coaxial design and consists of a cylindrical barrol held at ground potential, and an on-axis electrade which is held at a high voltage. A fast gas ralve pufis gas into the throat of the gun, which then breaks down in a uniform discharge. The resulting current ionizes the gas, creating a low temperature olasma. 
and produces an azimuthal magnetic field which provides the energy to launch a deflagration wave from the gun barrel. The magnetic field serves the same purpose as the chemical heat of combustion in a rocket engine, which drives a deflogration wave out the exhaust nozzle. This mode of operation differs from that of the Marshall gun, ${ }^{4}$ which is of similar construction, in that with the Marshall gun there is a localized current sheet behind the gas which acts as a magnetic piston to push the neutral gas and plasma out of the gun barrel. Thus, the Marshall gun mode is much like a chemical detonation, where neutral gas is driven and heated by a shock wave. With the deflagration gun, the plasma thermal energy remains low, whereas with the Marshall gun part of the magnetic energy goes into shock heating. The mode in which either gun operates depends on whether the voltage is applied to the center electrode before or after the gas arrives. If the gas arrives after the voltage is applied, the gun aperates in the deflagration mode; if the gas arrives before the voltage, the gun operates in the Marshall or snowplow mode, as it is called.

Te construction of the gun is shown in fig. 2.4. An earlier version was slightly longer, but with the same diameters. The major difference was that the center electrode was bent and brought out through the wall near the throat of the valve. This asymetry was removed with the design shown. The gun current was supplied by charging a $2.2 \mu \mathrm{F}$ capacitor, normally to $8 \mathrm{kV}$, as shown in fig. 2.1 , except that the $10 \Omega$ resistor was shorted. For the latar shots, the resistor was used to prevent large currents and lot the gun only serve 
as a spark trigger. The current had a quarter period of $3 \mu s e c$, and a peak current of $10 \mathrm{kA}$. Since the gun fire circuit was not crow-barred, the current rang through zero for several periods. During the first holf period the gun appeared to operate in a deflagration mode, but thereafter presumably in a snowplow mode, as has been observed by Cheng. Framing camera photographs of the gun plasma are given in the next chapter in fig. 3.4 .

Attempts mere made to crow-bar the gun current to keep the gun operating in the deflagration mode longer. Thase were not successful because of the gas valve $i \mathrm{itter}$. It was possible to pre-fire the gun fire ignitron and keep it conducting with a 1 k $\Omega$ ke日p-alive resistor, but it was difficult to time the crowbar trigger to coincide with the current maximum. Perhaps a multipulse trigger would have solved this problem, but was not tried.

\subsubsection{Timing}

The timing of the experiment was accomplished by use of a number of pulse distribution and variable-delay pulse amplifiers. The timing and sequence of avents for the two modes of operation are shown in fig. 2.6. Fig. 2.6(a) shows a diagram of the timing for the fused-bank mode where the plasma ginin is used only as a pre-ionizer. In this mode of operation it was necessary to trigger the oscilloscopes and diagnestics on a precurser because of the jitter in the pinch current start. The pinch current Rogowski loop proved to be a satisfactory 


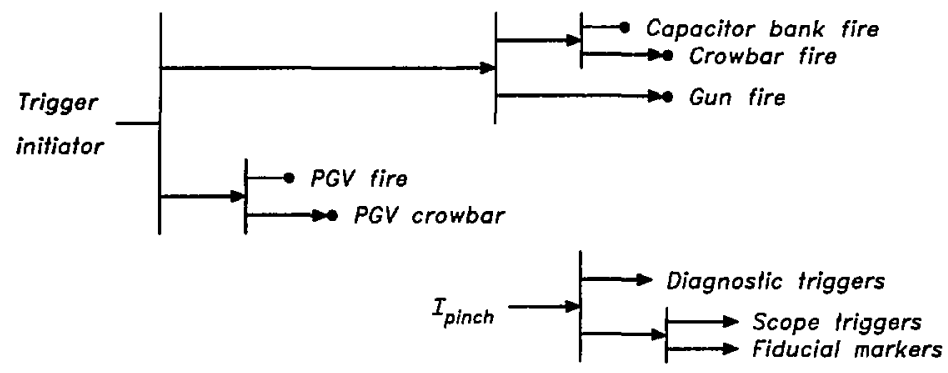

(a)

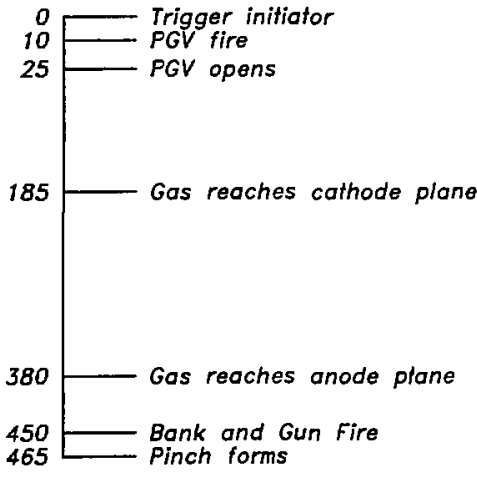

(b)

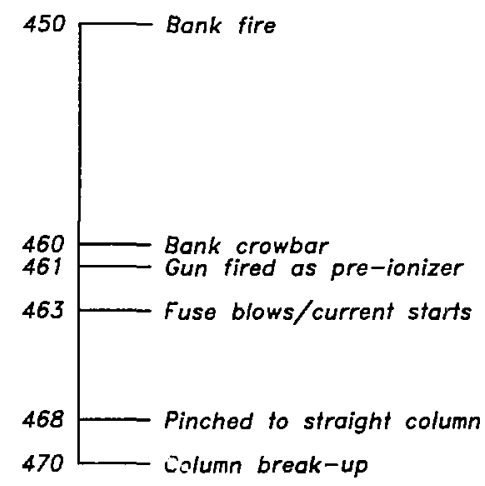

(c)

Figure 2.6. The timing and sequence of events for the FLR experiment. Timing for the fused-bank, no plasma gun mode is shown in (a). The vertical lines represent pulse distribution amplifiers, and the horizontai lines represent pulse delays. The arrowheads on the delay lines mean that the delay is variable. The dots represent ignition firing chassis. Parts (b) and (c) show the sequence of events, with (c) being an expanded time scale. Times are in usec. 
precurser and was applied to the input of a marker generator with the input voltage threshold set high enough to reject noise signals. The marker output was then used to trigger the scopes and diagnostics. In figs. 2.6(b) and (c) the sequence of events for the fused-bank mode is shown. The sequence of events for the gun mode of operation is not shown, but it is similar. The main difference is that the pinch bank and gun ignitrons were fired shortly before the gas arrived.

The equipment used for the timing wos at least fifteen years old and of vacuum tube design. It didn't have the low iitter times and shorter trigger delays of some of the newer designs, but it was fairly reliable. The timing control racks consisted of a coaxial cable patch panel, and the various delay and amplifier chassis. The variable delay chassis ${ }^{6}$ was dual channel, and had a minimum delay of $1.2 \mu$ sec and a maximum of $100 \mathrm{msec}$. Jitter was $\pm 0.1 \mu \mathrm{sec}$ in the fastest range. The pulse distribution amplifiers ${ }^{7}$ had a fanout of four, and had a pulse delay of order $0.1 \mu s e c$. The ignitrons were fired with a dual-ignitron-firing chassis, ${ }^{B}$ or a kip-tube chassis. ${ }^{9}$ The delay for the first was about $i \mu s e c$ and for the kip chossis less than $0.1 \mu s e c$. For events requiring ignitron firing ofter short delays, the kip-tube chassis was used. The marker generators'o were dual channel, had minimum delay (less than 50 nsec), had a 2000 volt output pulse, and had a variable pulse length which was determined by the length of an attached coaxial cable. The minimum pulse width was 10 nsec, and the maximum was limited only by the amount of line used. These generators 
were found to be very useful for comera and scope triggers, and for fiducial markers.

\subsection{Diagnostics}

A number of diagnostics were used on the experiment. The goals were to first observe the pinch structure and evolution, and then to measure the plasma parameters: the most important being the current and voltoge, and then the plasmo density, and density profiles, and finally, the plasma temperature. Several attempts were made to measure plasma temperature, but were unsuccessful. For this reason, plasma temperatures are inferred from the equilibrium conditions and the observed fluid velocities. Table 2.1 lists the diagnostics from which data were received. The UV monochromator is in parentheses since it was tried at the very end of the experiment, and there was not enough time available to get it working properly. The remainder of the chapter will be a discussion of each of these diagriostics.

\subsubsection{Framing cameras}

The use of a framing camera enabled observing the formatio. and decay of the pinch, and allowed measuring fluid velocities of the plasma. Framing cameras were used rather than streak cameras to allow 20 observation of the plasma at discrete times. The streak mode would have given better information on time bohavior and growth rates, but 
Tablo 2.1. Diagnostics for the finite Larmor radius Z-pinch experiment.

Type Measured

1. Framing camera

2. Laser interferometer

a. $\mathrm{He}-\mathrm{Ne}$

b. $\mathrm{CO}_{2}$

c. Argon-ion

3. X-ray scintillator/PM tube

4. Voltage probes
a. $V_{\text {bank }}$
b. Vanode

5. Rogowski loops

a. Bank current

b. Pinch current

c. Plasma gun current

6. Light pipe/PM tube

7. Gas arrival probe

(8. UV monochromator)
Three 10 ns frames for each shot

Electron density

Hard X-rays from pinch break-up Voltage vs time

Current vs time
Total visible output vs. time

Gas arrival timing

would hove lost in ease of interpretation since one spatial dimension is necessarily sacrificed to allow for a time scan. Iyo framing cameras were used, although not concurrently, for the entire set of shots. They were; (1) the TRW model 1D image-converter camera, and (2) the Imacon model 700 image converter camera, made by Hadland Photonics Limited. The TRW camera is commonly known as the STL camera, referring 
to Space Technology Laboratories, the original manufacturer. Both cameras have streak capabilities, but were not used in that mode.

The STL camera provided the most useful set of information, and was used with almost all of the shots. The most commonly used sweep voltage plug-in was the $6 \mathrm{~B}$, which provided three $17 \times 25 \mathrm{~mm}$ frames per shot. Exposure times could be set to 5, 10, or $20 \mathrm{nsec}$, and the deloy between frames could be independently set to $50,100,200$, or 500 nsec for each frame. The photacathode has an S-11 spectral response so that the images are an integral of the visible spectrum. The system gain is $2 x$, and final image resolution is 8 line pairs $/ \mathrm{mm}$. The gate on/gate off light extinction ratio is $10^{6}$, which was sufficient to block out increased electrode light ofter the experiment.

Care had be taken to align the image position properly, since the cathode gating grid left two shodows running lengthwise in the final image. It was necessary to adjust the comera position such that the grid wires did not block out the desired image. Since the grid wires are not on a focal plane, they behaved as if not entirely opague. The gain in their shadow was reduced, but not entirely to zero. This effect was observed in several shots where the plasma had an extended size.

The STL camera was mounted as close as possible to a side window of the vacuum chamber to allow use af as much light os possible. While in the focus mode, the image size was adjusted to make the pinch

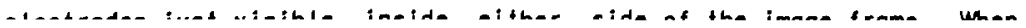


the laser interferometer was added it was necessary to raise the camera to the top window and view through a mirror. For these shots the original $\mathrm{f} 2$ objective lens was replaced with a $100 \mathrm{~mm} / \mathrm{f4.5}$ lens to maintain the some imoge size. There was, however, more than sufficient light with the argon plasmas. The light intensity was usually reduced with a combination of neutrol density filters, and by closing down the iens aperature. (With the hydrogen plasmos there was still sufficient light, except that the electrodes were usually brighter than the plasma. Efforts to mask out the electrodes to look only at the plasma were not entirely successful.)

The image on the anode phosphor was focused by a $f 1.2$ transfer lens onto a film plane. Poloroid type $57 \mathrm{film}$, which hos a speed of 3000 ASA, wos used for most of the shots. Where higher dynamic range was desired, and to provide the capability to be scanned, Kodak Royal $x$ par, film wos also used. It has a 1250 ASA speed, which can be doubled of development time, provided the dynamic range can be sacrificed. For protection, all the Polaroid prints were coated with a sproy laquer.

The Imacon comera was used with the $2 \times 10^{7}$ frames/sec plug-in. It provided ight to sixteen fromes seporoted $50 \mathrm{nsec}$ with $10 \mathrm{nsec}$ exposures. The image size is $16 \mathrm{~mm} \times 18 \mathrm{~mm}$ with eight frames ond $8 \mathrm{~mm} \times 18 \mathrm{~mm}$ with sixteen fromes. The imoge resolution is 5 line pairs/mm, and the system gain is $2 x$. The image was recorded on either Polaroid type 47 or type $410 \mathrm{fi} \mathrm{Im}$. 
The moin difficulty with the Imacon was its irability to effectively block out light after the scan. The aperture stops of the objective lens and the neutral density filters had to be carefully odjusted to prevent a bright blur over the entire image plane. Even then, the film had a background fog which reduced the available dynomic range, and resolution. For these reasons the camera was only used on a small number of shots.

In order to understand the relationship of the framing camera photographs to the plasma density and temperature, the emitted light intensify for an argon plasma as a function of temperature was calculated. At lower temperafures, spectrol line radiation and recombination radiation are important, and of higher temperatures electron-ion bremsstrahlung is important. All three of these mechanisms are dependent on the ionization state, or average charge $Z_{\text {ave }}$ of the ion, which wos colculated as a function of temperature by the technique discussed in appendix $C$.

The spectrol line infensity was determined by suming the intensities of individual lines. Expressing in units of radiated power per unit volume, the intensify is

$$
P_{i}=\sum_{i} n \nu_{i}^{k} A_{i}^{k \prime} n^{k} .
$$


$k^{\prime h}$ to the $1^{\text {th }}$ excited level, $A^{k} f^{\prime}$ is the fransistion probability (units $\left.\sec ^{-i}\right)$, and $n_{i}^{k}$ the density of ions which have on electron in the $k^{t h}$ excitad level. The subscript $i$ denotes the particular line. Since there are several hundred argon lines in the visible, only the strongest lines, and only those in a wovelength range of 0.4 to $0.5 \mu \mathrm{m}$ were included. To be consistent, the bremsstrahlung ond recombinotion radiation were also limited to this range.

The population of the excited levels is related to the density of the ground stale by the Boltzmann formula.

$$
n^{k}=n^{\prime} \frac{g_{k}}{g_{1}} \frac{e^{-E_{k} / k T}}{\mathrm{e}^{-E_{1} / k T_{0}}}
$$

Here $g_{k}$ and $g_{1}$ are the degeneracies, and $E_{k}$ and $E_{l}$ are the energies of the leveis $k$ and 1 . The population of the ground levels of each ionization state are appraximated by gaussian distributions with the overoge charge as an independent voriable. Such profiles closely motch those calculated by House. 11 The density of the $i^{\text {th }}$ ionization state is then

$$
n_{i}\left(Z_{a v}\right)=n_{t} \exp \left(-a\left(Z_{i}-Z_{a v}\right)^{2}\right)
$$

where $n$, is the total number density of ions and neutrals, and $z_{\text {ave }}$ is a continuous function of temperature, and is calculated by the technique shown in appendix $C$. The constant $a$ is chosen such that 
$n_{1}\left(z_{1}+1 / 2\right)=1 / 2$. Note that the definition of the subscript $i$ has been changed here to indicate the $i^{\text {th }}$ ionization state. The values of g. $E, A$, and the wavelength, are tabulated by Wiese, ef all2 for noutral and singly ionized otoms. For doubly and more highly ionized ions the tronsistion probabilities and degeneracies of the visible lines are not available. However, for doubly ionized argon they can be estimatod by comparing the relative intensities given by Striganov and Sventitskiil3 with those in the ultroviolet for which these parameters are known.

The frequency dependent expressions for the bremsstrahlung and the recombination radiation are given by Cooper ${ }^{14}$ in the hydrogenic ion approximation. That is, the ionization potential of an ion with a charge $Z e$ is $Z^{2} E_{H}$, and the energy of the excited level $k$ is $Z^{2} E_{H}\left(1-1 / k^{2}\right)$, where $E_{H}$ is the ionization potential of hydrogen. The intensity of the bremsstrahlung radiation is

$$
P_{b}(\nu)=1.70 \times 10^{-40} n_{i} n_{0} Z^{2}\left(\frac{E_{H}}{T_{0}}\right)^{1 / 2} \exp \left(-\frac{h \nu}{k T_{0}}\right) .
$$

The units are ergs/(sec $\left.\mathrm{cm}^{3}\right)$ for the intensity, with $T_{\text {, and }} E_{H}$ givan in ev. A similar expression for the recombination radiation is 


$$
P_{r}(\nu)=c n_{\bullet} n_{i} Z^{4}\left(\frac{E_{H}}{T_{\bullet}}\right)^{3 / 2} \exp \left(-\frac{h \nu}{k T_{0}}\right) \sum_{q \geq\left(\frac{Z^{2} E_{H}}{h \nu}\right)^{1 / 2}}^{\infty}-\frac{1}{q^{3}} \exp \left(\frac{Z^{2} E_{H}}{q^{2} T_{\theta}}\right)^{\prime}
$$

where $c$ is a constant equal to $3.40 \times 10^{-40}$. The units are the same as those in the previous equation.

The intensities for argon from these three mechanisms were then integrated over the frequencies corresponding to the 0.4 to $0.5 \mu \mathrm{m}$ wavelength range used for the line radiation, and were plotted, as is shown in fig. 2.7. At low temperatures, the line radiation dominates, and at higher temperatures the bremsstrahlung does. The line intensity falls rapidly after $6 \mathrm{eV}$ because the strongest lines are in the ultraviolet and x-ray spectrum for the more highly ionized ions. The dip in the total light is probably not as pronounced in a real plasma as is shown in the graph. Line intensities for higher level transistions, which are known to exist, are not included since data for them is not available. (Striganov and Sventitskii, 13 for example, Iist sixteen visible lines for triply ionized argon for which no doto other than the wavelength is given.)

The other interesting feature is that the bremsstrahlung radiation falls off gradually with increasing temperature, despite the shorply increasing average ion charge. However it is necessory to remember that the camera detects only the visible radiation. The total 


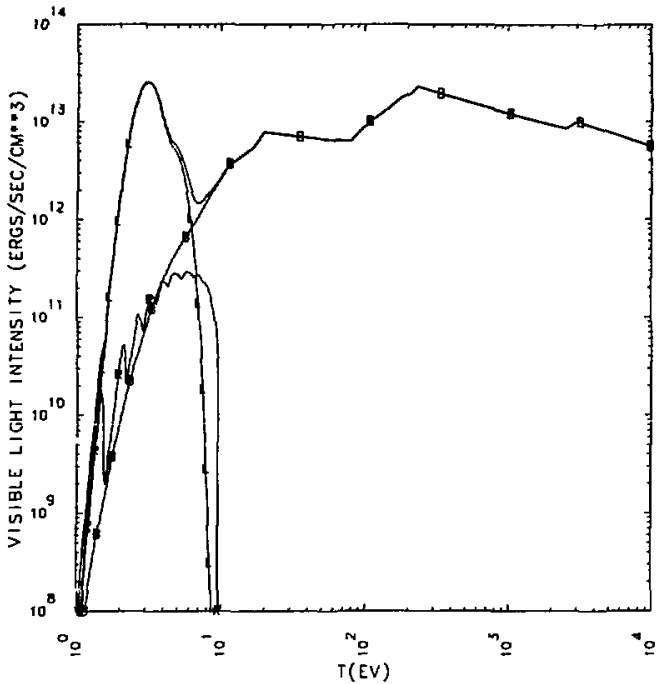

Figure 2.7. Calculated visible radiation of an argon plasma in 00.4 to $0.5 \mathrm{\mu m}$ wavelength window, as a function of temperature, for $n=10^{18} \mathrm{~cm}^{-3}$. The curves are marked B for bremsstrahlung, $\mathrm{L}$ for line radiation, and $R$ for recombination radiation. The unmarked curve is the sum.

bremsstrahlung radiation increases with increasing temperature and ion chorge as $Z^{2} T_{1 / 2}^{1 / 2}$, but the moximum is shifted to the ultraviolot sufficiently that the intensity drops in the visible. This effect is shown by a plot in Cooper's paper. ${ }^{15}$ 
Thus, to conclude the discussion of the plasma radiation, the light emitted from an argon plosma rises sharply to a maximum at about $3 \circ v$, and then stays relatively constant (probably to within a factor of five) as higher temperatures are attained, given a fixed particle density. For fixed temperatures, the intensity varies linearly with the density at lower temperatures, provided the excited states are in local thermodynamic equilibrium (LTE), and as the square of the density of higher temperatures, as can be seen in eqs. (2.1) and (2.4). If the plasma is not in LTE at low temperatures, then the line intensity is also proportional to the square of the density. If the density is sufficiently low the excitation process cannot keep up with the radiative decay. Thus, a dark region on the framing camero image must mean that either the electron temperature is below $2 \mathrm{eV}$, or that the density has decreosed. It will not indicate a region of higher temperature, however.

Before concluding the discussion of the framing cameras, it is necessary to mention a system that was useful in analyzing the framing camero photographs, and that is the PDS photodensitometer which is operated by the Technical Photography group at LLNL. This system has the capability of digitizing an $x-y$ scan with up to 13 million points, with a resolution of anywhere from 4 to $200 \mu \mathrm{m}$. This dato is read onto a magnetic tape, which may be used to transfer the scan to the laboratory's computer system. where software is available for processing the data. Routines are available from Trimble, 16 which can

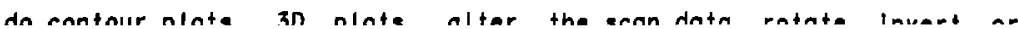


Fourier transform and filter the scans. The only drowback to the system is that the densitometer can only scan transparencies. For this reason only the photographs on the Royal $X$ pan $f i l m$ were scanned.

\subsubsection{Laser Interferometers}

Measuraments of the Z-pinch electron density and density profile were made with a spatially rosolved Mach-Zehnder laser interferometer. A diagram of the set-up is shown in fig. 2.8. Rather than use a pulsed laser, a CW (cantinuous wave) laser was used. Time resolution and light gain was obtained by gating a micro-channel plate intensifier with a $10 \mathrm{nsec}$ pulse. The gated image was recorded by a silicon Intensifier Target (SIT) tube TV camera and displayed on a TV monitor. The image was permanently recorded by photographing the monitor image. The use of short gate times is possible becouse of the high gain of the intensifier and SIT tube combination; it being $10^{4}$ times more sensitive than the standard vidicon.

The construction of the system was relatively simple since mechanical vibration is non-existent for the short exposure times used. The beam splitters and mirrors were $75 \mathrm{~mm}$ diameter and flat to $\lambda / 10$. The vacuum windows were not optically flat because of the susceptibility to damage. However, they were selected to introduce the least amount of distortion. The plasma light was filtered out by spatial filtering, and by using narrow-band-transmission interference filters. 


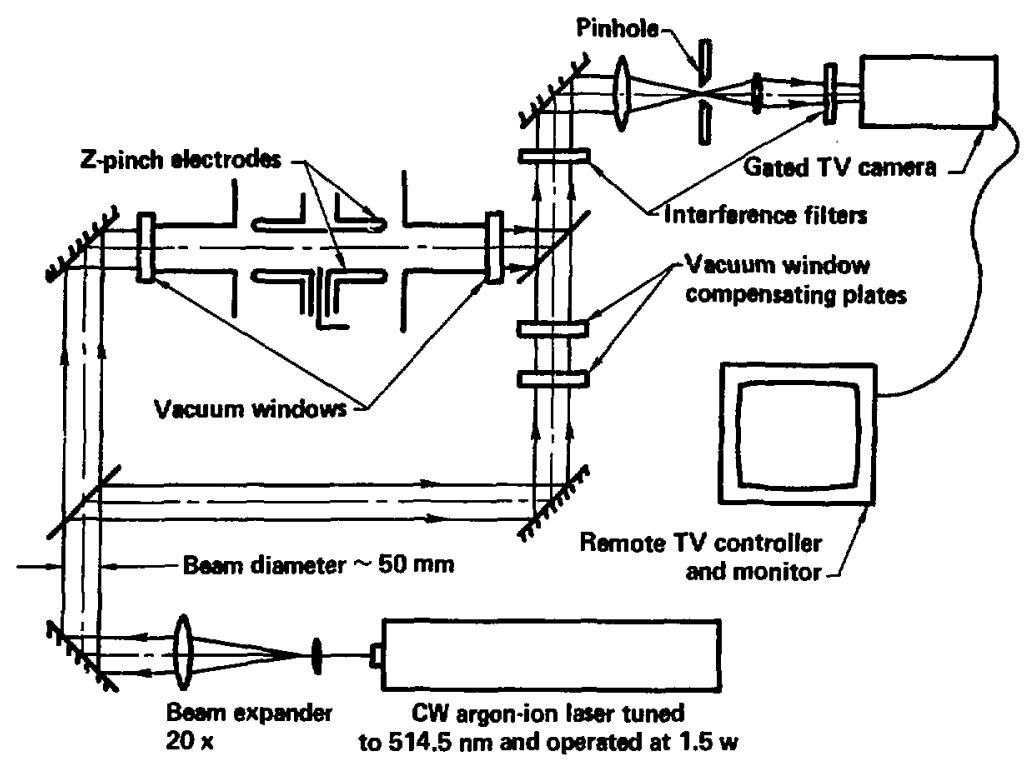

Figure 2.8. Diagram of the Mach-Zehnder laser inferferometer used to measure electron densities and density profiles. The TV camera consists of a micro channel plate intensifier and SIT tube. The intensifier is gated by a 10 nsec pulse.

The advantages of the system are that the alignment is easier with a CW laser, and that data recording with Polaroid film is much lesa complicated than exposing and developing the film plates used in puleed interterometry. There are, however, several disadvantoges with this systom. First, it is not possible to use double pulse hologrophic 
techniques. Therefore, it is necessary to use optically flat elements within the interferometer. Unfortunately. the vacuum windows are coated with and damaged by electrode debris with each shot, making frequent replacement necessary to obtain good interferograms. Second, the resolution of the final image is also limited by the resolution of the intensifier/SIT tube/TV monitor combination. The nominal resolution of the micro channel plote is 10 line pairs/mm. This resolution would be acceptable except that the beam size had to be reduced $5 x$ to maintain sufficient intensity, and to obtain the proper image size on the monitor. Thus, the resolution in the scale of the pinch is only 2 line pairs/mm. The poor resolution could be corrected by using a smaller beam diameter through the pinch, but nevertheless it is still a limitation. Third, for many of the higher density shots, the plasma light within the wavelength band of the filter was as bright as the laser illumination.

A $60 \mathrm{~mW} \mathrm{CWHe-Ne} \mathrm{laser,} \mathrm{the} \mathrm{first} \mathrm{laser} \mathrm{used,} \mathrm{gave} \mathrm{a} \mathrm{number} \mathrm{of}$ good density profiles. With this laser, it was necessary to use 50 nsec exposure times because of the low power. There was also trouble with too much plosma light, and with a lack of sensitivity at lower line densities, and with lower $Z$ materials. The fringe shift $N$ of an interferometer can be shown to be proportional to the wavelength $\lambda$ of the laser, times the line integral of the electron density, 


$$
N(y)=\frac{\lambda}{2.3 \times 10^{13} \mathrm{~cm}^{-2}} \int_{0}^{L(y)} n_{0}(x, y) d x
$$

where $x$ and $y$ describe the coordinate position, and $L(y)$ is the thickness of the plasma.

With the motivation of $17 x$ greater sensitivity, and with the hope of improving the resolution of the interferogram, it was decided to use a pulsed TEA $\mathrm{CO}_{2}$ laser of the $10.6 \mu \mathrm{m}$ wave length. The system was built with a $1 \mathrm{~J}, 70 \mathrm{nsec}$ laser and with the same interferometer configuration, except that $\mathrm{NaCl}$ and Ge optics were used. The detection system was also raplaced by films sensitive to the $10.6 \mu \mathrm{m}$ radiotion.17-19 However, in opting to use the system, the negative index of refraction of the plasmo was ignored. The plasma acted like a diverging cylindrical lens, refracting the beam enough that it was not defectable at the film plane. It was not possible to correct for the divergence since the index of refraction is a function of the electron density, which varies with both time and position.

For these reasons, a CW argon-ion laser operated at $514.5 \mathrm{~nm}$ was substituted far the $\mathrm{CO}_{2}$ laser for the last four weeks of operation. The argon-ion laser was used because of its higher $\mathrm{CW}$ power, and because of availability. Its major drawback is that with aigon, the plasma has optical emission lines at every wavelength the laser does. Therefore, oven with the highar power level of the argon laser, it was 
not possible to filter out the plasma light. The problem was less severe when using other gases.

Density profilos were obtained from Abel inversions of the interferograms. Appendix $B$ contains a discussion of this technique and the scaling and limitations of the laser interferogram measurements.

\subsection{3. $x$-ray measurements}

Measuremenis of $x$-ray emission with energies $\geq 1 \mathrm{keV}$ were made with a scintillotor/photomultiplier tube combination. Attempts were also made to obtain i-ray pinhole photographs during the last week of the experiment, but these were unsuccessful.

The first measurements were with a NoI(TI) scintillator. This crystal has a $1 / e$ decay tine of 250 nsec. Fer later shots a plastic scintillator was used to decrease the decay time to 2 nsec, and hence increase the frequency response. Both scintillators were located at the chamber wcll and behind a thin (.0006") Al foil.

The photomultiplier, on RCA 6810A, was mounred directly behind the scintillator. It has an S-11 photo-response, and operated with a negative 2000 valt cathade potential while the anode was held at ground potential. Tre last four dynode stages were connected by capacitors of sufficient size that the phatomultiplier could sustain 1 volf across $50 \Omega$ for $0.2 \mu$ sec without any linearity loss. 
Energy measurements were made by delermining the absorption through Al folls of different thickness. These foils were plaied in the vacuum and in front of the scintillator. Neutral density filters were sandwiched in between the scintillator and photomultiplier to prevent saturation. There was some fluctuation $(\sim 10 \%)$ in the signal height for the different shots, however. This required averaging over several shots for each foil thickness. The data points were plotted on a logarithmic scale, and fitted to a straight line, the slope of which defermined the mass absorption coefficient. These coefficients were then compared with absorption coefficients given by Osher 20 to obtain $x$-ray energy.

By fitting the data to a straight line it is implicitly assumed that the $x$-rays are mono-energetic. If the relative intensity is not logarithmically linear in the absorber thickness, but rather has a positive $2^{\text {nd }}$ derivative, then one must conclude that a spectrum of energies is being observed. Then it is necessory to follow the analysis of Jahoda, et $a,^{21}$ by integrating over the energy spectrum, while weighting the integrand with the absorption per unit energy. The energy spectrum is determined from bremsstrahlung radiation using a Maxwell distribution of electron velocities. The distribution is Maxwellian if the electron self-collision time is smaller than the experimental time scale, which it is by a factor of $10^{3}$ for this experiment. With this technique, the total absorption, as a function of absorber thickness, can be calculated for a given electron temoerafure. 


\subsubsection{Other meosurements}

The bank current, the pinch current, and the gun current were measured with Rogowski loops. These were hand-constructed by stripping back and removing the outer conductor of RG-58 coaxial cable approximotely $40 \mathrm{~cm}$, removing $20 \mathrm{~cm}$ of dielectric from the center conductor, winding the center conductor aack on itself, and then soldering it to the outer conductor. The end was then wound into one turn, and placed around the conductor to be measured.

The Rogowski coil output, which is proportional to the derivative of the current, was integrated with a passive RC network to provide a signal proportional to the current. The capacitor was chosen to be large enough that the integrator remained linear for times of interest. The loops were calibrated by comporing their output with a coil of known dimensions and turns.

Pinch anode and fuse voltages were measured with resistive voltage dividers each af which consisted of a string of 10 $500 \Omega / 2$ watt carbon resistors, yielding a voltage reduction of $100: 1$. The resistors in the divider were snall enough that stray capacitance did not affect the frequency response. The resolution of the anode voltage was 10 nsec or better.

The total light output as o function of time was monitored with a light pipe connected to an RCA 1P21 photomultiplier tube, which has an 5-4 photo-response. No offorts were made to collimate the light into 
the end of the light pipe; it wos simply pointed in the general direction of the pincin.

The gas arrival time was measured in the throat of the gas gun approximately $5 \mathrm{~mm}$ from the cathode plane. A ceramic insulated probe. which was bare at the end, wos inserted perpendicular to the axis of the opening and biased it with a small capacitor charged to $2 \mathrm{kV}$. When the gas arrived there was an electrical breakdown from the probe to the wall, which discharged the capacitor. The signal was the voltage drop across a $50 \Omega$ resistor in series with the capacitor. The capacitor was chosen to give a $10 \mu \mathrm{sec}$ pulse width, and a charge resisto was chosen large enough that the capacitor could not recharge until after the event. This signal enabled adjusting the bank timing to coincide with the gas arrival.

Attempts were also made to look af near-UV line spectra with a McPherson $1 / 3 \mathrm{~m}$. 12 monochromator, with the hope of obtaining electron temperature masurements. The monochromator was located beneath the pinch chamber, with the pinch imaged lengthwise along the entrance slit. It was converled to a narrow bond spectrograph by removing the oxit slit and focusing the entrance slit image on a film plane. UV grade quartz optics were used throughout to allow use down to $185 \mathrm{~nm}$ wavelength. Both Polaroid type 57 and Kadak Royal $x$ pan films were usod and were faund ta be sensitive dawn to $250 \mathrm{~nm}$. Calibration was done with a $\mathrm{Hg}$ arc lamp. The goal was ta do several shots in the spectrograph mode and find a higher ianization-level spectral line 
which did nol havs any close neighbors, and to then re-insert the slit and on subsequent shots observe the time evolution of the line intensity with a photomultipliar. However, in looking for lines, all that could be observed was a continuum. For this reason, this diognostic was not pursued.

References for chapter 2

'D. Y. Cheng, Nucl. Fusion 10, 305(1970).

${ }^{2}$ There is no reference available for this gas valve. It is similiar in design to the Marshall gun gas valve (see ref 5 ), in that there is an electromagnetic hamer which drives a steel rod off an O-ring seal.

${ }^{3}$ Cheng. loc. eit., and C. N. Chang, D. C. Johnson, D. Y. Cheng. and M. D. Smith, "A Micro-second Response High Pressure Valve", Plasma Research Laboratory. University of Santa Clara.

theng. loc. cit.

$5 \mathrm{~J}$. Marshall, Proceedings of the Second UN International Conferance on the Peoceful uses of Atomic Energy, Vol. 31, p. 341.

${ }^{6}$ Lawrence Livermore Laboratory Electronics Engineering Dept. schematic LE 8666-1C. 


\begin{abstract}
'Lowrence Livermore Laboratory Electronics Engineering Dept. schematic LE 1623-1E.
\end{abstract}

- Lawrence Livermore Laboratory Electronics Engineering Dept. schematic LE 3299-1A.

${ }^{9}$ Lawrence Livermore Laborotory Electronics Engineering Dept. schematic LE 9395-1A.

${ }^{10}$ Lawrence Livermore Laboratory Electronics Engineering Dept. schematic LE $10649-1 \mathrm{M}$.

${ }^{11}$ L. L. House, Astrophy. J. Suppl. Ser. 18, 307 (1964).

12w. L. Wiese, M. W. Smith, and B. M. Miles, Atomic Transistion Probabilities (Not. Bur. Stand., Washington, 1969). Vol. 2, Pp. $187-224$.

13A. R. Striganov, and N. S. Sventitski i, Tables or Spectral Lines of Neutrol and Ionized Atoms (IFI/Plenum, New York, 1968), Pp. 377-386.

14J. Cooper, Rep. Prog. Phys. 29, 35 (1966).

${ }^{15}$ Cooper, Ibid.. p. 87.

${ }^{16} \mathrm{~B}$. Trimble, Technical Phatography Group, LLNL (private communicotion).

17J. Manning, and Q. KIingler, "Kalvar Film Report", EGeG Report No. EGG1183-5054, 1974. 
18p. R. Foreman, S. Humphries, Jr., and R. W. Peterson, App 1. Phys. Lett. 22, 537(1973).

${ }^{19}$ G. R. Mitchel, B. Grek, T. W. Johnston, F. Mortin, H. Pépin, "Nanosecond Photography af $10.6 \mathrm{\mu m}$ Using Silver Halide Film", INRS-Energie Reporf PL-122 (Varennes, Quebec, Conada), 1978.

$20 \mathrm{~J}$. E. Osher, in Plasma Diagnostic Techniques, edited by R. H. Huddlestone, and S. L. Leonard (Academic, New York, 1965), p. 584 .

${ }^{21}$ F. C. Jahoda, E. M. Little, W. E. Quinn, G. A.Sawyer, and T. F. Stratton, Phys. Rev. 119, 843 (1960). 
CHAPTER 3

The Z-pinch Formation

3.1. Pinch formation

3.1.1. Gos flow into the chomber

In the last chapter, and in appendix $A$, the gas valve and its output as a function of time are discussed, however, the distribution of the gas as it enters the chamber is ignored. In this section the gas distribution, the breakdown process, and formation of the pinch is discussed. A small number of shots were initiated with a deflagration 
plasma gun. A discussion of the formation of these types of pinches is doferred to the noxt section, however.

As the gas valve opens, the gas trovels through the various orifices and intermediate regions of sonic velocities, as is discussed in appendix A. (See figs. 2.3 and 2.4 for the geometry.) This behovior is a result of the theory of flow through nozzles, where it is found that the flow is sonic if the pressure drop across the nozzle is greater than a factor of two, and that a sonic flow through the constriction af the nozzle becomes supersonic of the diverging end.1,2 Similarly, with the Z-pinch geometry, as the gas enters the vocuum region between the pinch electrodes, it exponds and becomes supersonic.

The phenomenon of a steady-state free-jet expansion into a vacuum has been studied extensively in the confext of supersonic wind-tunnels, molecular beam sources, and rocket exhaust plumes in the upper atmosphere. The flow of gas from the gas valve plenum to the pinch chamber is not a steady state process. However, many properties of the flow can be deduced by considering the steady-state results, after which non-steady state effects will be examined.

The general features of the jet, and the density of the core region as a function of the distance from the orifice are shown in fig. 3.1. An isentropic part of the flow appears to pass from the opening to the chamber through a barrel, the sides of which ore the shock wave formed by the coalescence of compression waves from the jet boundary. "These waves originafe af the boundary as reflections of the 

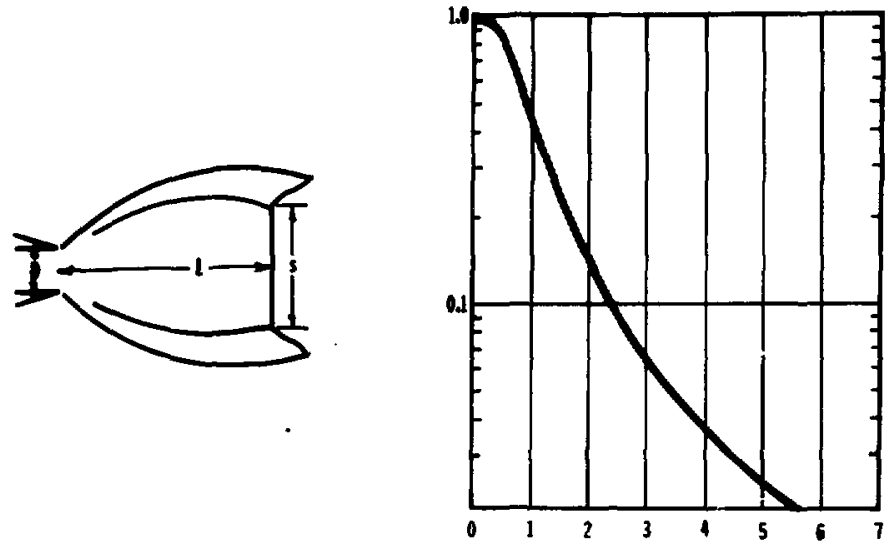

(a)

(b)

Figure 3.1. Free jet expansion into the vacuum chamber. (a) Geometry of the ief. (b) Density and pressure variation of the core region from the Owen-Thornhill theory. 3

initial expansion fan. The bottom of the barrel is the Mach intersection of the lateral shock waves, and is called the Mach disk, or the Riemann wave. The strength of the shocks is related to the back pressure of the pinch chamber. In the limit of a perfect vacuum the shocks cease to exist. As the back pressure approaches the jet pressure, the shock waves become stronger.

The isentropic core was first described theorelically by owen and Thornhill3 who used a numerical method of characteristics solution. Their results were verified experimentally by many others. A 
particularly exhaustive study was done by Love, ot al. 5 who both experimentally and theorefically studied such aspects of the problem as the initial angle of the iet, the location of the shock boundaries, and the size and location of the Mach disk. These parameters were found to vary mainly with the mach number at the orifice, and the ratio of the orifice pressure to the background pressure. The papers by Sherman, 4 and Ashkenas and Sherman ${ }^{6}$ give experimental data on the density of the shock regions, as well as to verify mony of the predictions of Love, ef 01.5

Considering the flow through the orifices in the plasma gun anode (see fig. 2.4) as sonic, it is possible to determine the flow parameters at the cathode plane by using mass continuity, the Euler equation, and the isentropic equation of state. Thus, it is found that at the entrance to the chamber the jet hos a Mach number of 4 , and that the rotio of the jet pressure to the chamber pressure is $10^{4}$ to $10^{5}$, based on a $10^{-4}$ Torr chamber vacuum. The maan free pafh for collisians at the orifice is $3 \times 10^{-3} \mathrm{~cm}$, with a one atmosphere plenum pressure. Thus the inudsen number, which is the ratia of the mean free poth to the ief orifice diameter, in this case, is $\sim 2 \times 10^{-3}$ at the cathode, and $<2 \times 10^{-1}$ at the anode. For Knudsen numbers $\ll 1$, a gas is collisional, and a continuum theory is valid.

As the gas leaves the cathode plane it expands into a fan similar to that shown in fig. 3.1(a). Its initial angle is $65 \pm 10^{\circ}$ from the axis. The distance to the Mach disk is $\sim 60$ times the diameter of the 
jet orifice $d$, which is wall behind the anode. The density and pressure on-axis fall off as shown in fig. 3.1(b). For distances $x / d$ greater than 2, the pressure drops as $(x / d)^{-2}$, where $x$ is measured from a point slightly downstream from the orifice. Because of the adiabatic equation of state, the densify falls off as $(x / d)^{-2 / \gamma}$, where $\gamma$ is the ratio of the specific heat capacities. Thus, for argon, the density falls off as $(x / d)^{-6 / 5}$. In the radial direction, the density and pressure vary as $\cos ^{2} \theta$, where $\theta$ is measured from the axis about a pivot at the cathode plane. This variation from the axis is valid up to the point where the local pressure equals the background pressure. At that point there is a shock, and a higher densify region. For small back pressures, the shock region is well away from the axis, hos a low density, and therefore need not be considered. Fig. 3.2 shows a contour plot of this scaling in the Z-pinch geometry. The off-axis scaling is only valid for $x / d \geq 1$, since the orifice geometry has a much lorger effect there. The axial variotion does correspond to the Owen-Thornhill calculations, however. The contours for $x / d<1$ are not calculated values, but are estimated by smoothing from the orifice density to the density in the expanded jet. The general features of the jef are apparent, however. The shock region would then be located at the uniform density confour where the vocuum pressure equals the jet pressure.

A good experimental example of this barrel shock structure is shown in the interferogram in fig. 3.12. The photograph was taken after the pinch current had started, for a shot with a higher back 


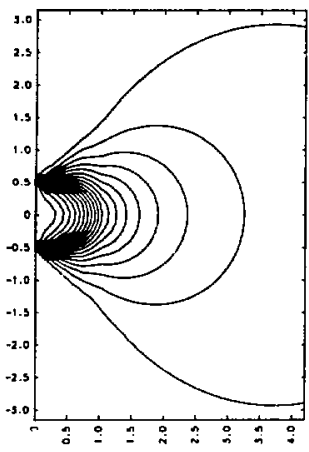

(a)

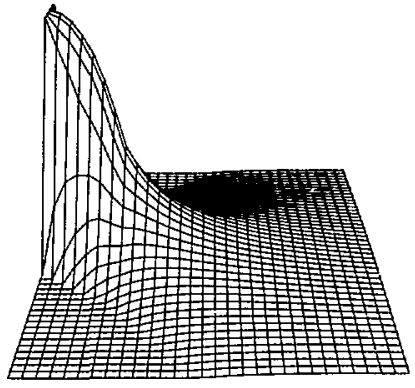

(b)

Figure 3.2. (a) Contour and (b) $3 D$ plots of the density profiles from the Owen-Thornhill theory. The units are orifice diameters. The cathode is on the left, and the anode is on the right.

pressure. Because of a pinhole used in the optics, the image has a Schlieren effect, ie., regions of large gradients in the plasma density are seen as dark lines running across the image.

Up to this point the position of the anode has been ignored. Being located only 4.2 orifice diameters downstream, it certainly affects the flow. The question is what happens to the gas ofter it sirikes the ancde. If the flow at the anode is molecular, i.e., if it has a high Knudsen number, then the gas particles would rebound from the anode at all angles, and increase the backpressure at all positions between the lectrodes. The Knudsen number is low, however. Thus the gos is collisional, and puddles against the electrode, escaping in a 
more radial direction, and not affecting the flow upstream more than several mean free paths away. This effect is verified numerically by Bird ${ }^{7}$ in considering the time evolution a freely expanding jet impacting on a wall. He does find, however, that at later times there is a reflected shock which moves taward the orifice, radically changing the character of the jet. Typically, the pinch bank was fired before this structure could develop further upstream, however.

Two other effects are related to the non-steady state gas distribution. (1) Because of the short time between when the gas enters the chamber, and when the pinch bank is fired, the radial variation of the gas cannot attain the $\cos ^{2} \theta$ distribution. Rather, the gas distribution can only extend a distance corresponding to the local flow velocity, times the time from when the gas ïirst enters the chamber. The density is then expected to be zero past one of the constant density profiles shown in fig. 3.2(a). The position of the limiting profile depends on the time of the bank firing, however. Because the pressure in the orifice is a steeply varying function of time, the axial variation of the pressure is necessarily steeper than the $(x / d)^{-2}$ variation since the pressure of the gos entering the chamber at later times is higher. Fig. A.5 of appendix A shows the pressure in the intermediate chamber to rise a factor of ten in $150 \mu \mathrm{sec}$, stay level for about $100 \mu \mathrm{sec}$, and then fall off almost as rapidy as it rises. The pressurg at the orifice is expected to have the same variation. The magnitude of this effect is of course also 


\subsubsection{Gas breakdown}

The breakdown characteristics and the Paschen curves for argon, and the noble gases are well-known and available. ${ }^{8}$ The breakdown valtages for the noble gases are much lower than those of other gases. The minimun breakdown voltage for both aigon and krypton is 230 volts, with a Pd (pressure times gap distance) of 1 Torr-cm. For our geometry this is a number density of $7 \times 10^{15} \mathrm{~cm}^{-3}$. At both lower and higher pressures the breakdown voltage is much higher. With $4 \mathrm{kV}$ across the pinch electrodes, which was olso the most commonly used bank charge voltage, the Paschen curve shows an upper 1 imit of $2 \times 10^{18} \mathrm{~cm}^{-3}$, and a lower limit of $1 \times 10^{15} \mathrm{~cm}^{-3}$ on the gas density. That is, breakdown is possible of any density between $10^{15}$ to $10^{18} \mathrm{~cm}^{-3}$. The anode voltage is not instantaneously $4 \mathrm{kV}$, but $r$ ises at a rate of $40 \mathrm{kV} / \mu \mathrm{sec}$. Therefore, as the anode volfage rises, the range of densities over which breakdown may occur, increases from a small range about the Paschen minimum, to a range spanning over three orders of mogn itude.

From the gas valve calculations, using a one atmosphere plenum fill pressure, and from the curve in fig. 3.1, the on-axis density is estimated to be 5 to $10 \times 10^{15} \mathrm{~cm}^{-3}$ at the anode. The value is of course very dependent on the timing. The path of breakdown is not observed to be perpendicular to the electrodes, but rather follows the gas distribution. Shots with the camera timed early show the initial current to be fon-, or borrel-shaped, with the distribution very 
similar to that shown in figs. 3.1 and 3.2. An example of one of these shots is shown in fig. 3.3. There is also evidence that the initial current poth is af the edge of the pinch. The light emission in the darker fan-shaped regions, as is seen in the framing camera images, is the brightest on the edges. This effect is seen in figs. 3.10,3.11, 3.12, and 3.14 of the next section. The interferogram and Abel-inverted density profile of fig. 3.12 show the density to be mainly in a small region near the edge, as would be expected in a

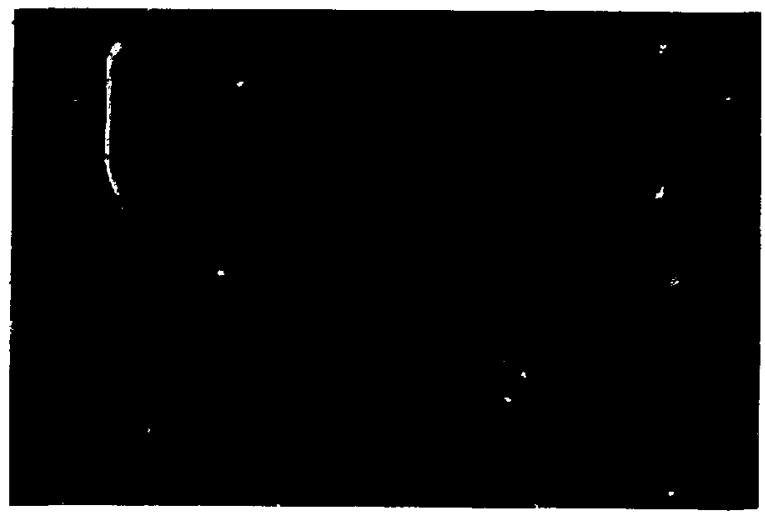

Figure 3.3. Framing camera photograph of on early stage of the pinch formation. The pinch current is $40 \mathrm{kA}$, and the electrode separation is $23 \mathrm{~mm}$.

snow-plow type collapse of the pinch. 


\subsubsection{Pinch formation}

After the gas breakdown, the plasma column contracts towards the axis, often farming a straight, long-lasting column. The time for the plasma to collapse, the pinch time $t_{p}$, was found to vary with the ges valve plenum pressure, and the deloy to bonk fire. The results of measurements from a number of shots are shown in fig. 3.4. The time to pinch was measured from the onset of the pinch current to the time of the voltage spike and current dip, which usually corresponded to the time that the entire column had pinched. That the pinch time should vary with the delay to bank fire is reasonable, since for longer delays the gas is allowed to expand a longer time in a radial direction between the electrodes, and hence has further to go when the pinching begins. The effect also depends on the time behovior of the gas density. How the gas density and initial radius are related to the pinch time is discussed in the following paragraphs.

The pinch time can be predicted by the well-known snowplow model, 9.10 given the initial radius and mass density of the plasma, and the current as a function of time. Consider a force balance between an infinitesimally thin current carrying shell and the magnetic field pressure.

$$
\frac{d}{d t}\left(m \frac{d r}{d t}\right)=-\frac{B^{2}}{2 \mu_{0}} 2 \pi r
$$




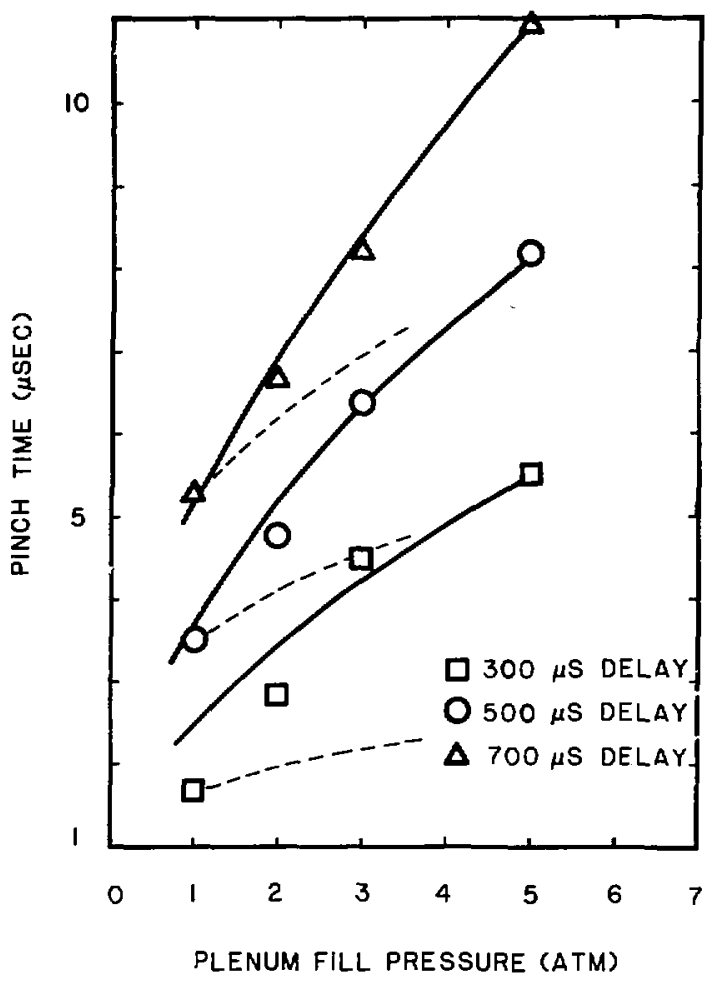

Figure 3.4. Meosured time to pinch $t_{p}$ vorsus the pulsed gos valve fill pressure, for three separate bank-fire delay times. The lines show the scaling with the density. The solid line is $t_{p} \alpha_{\rho}^{1 / 2}$, and the dashed line is $t_{\rho} \propto \rho_{0}^{1 / 4}$. 


$$
m(r)=\rho_{0} \pi\left(R_{0}^{2}-r^{2}\right)
$$

The mognetic field $B$ is also a function of $r$ and $t$.

$$
\theta(r, \rho)=\frac{\mu_{0} I(t)}{2 \pi r}
$$

For these last three equations $r$ is the pinch radius, $R_{0}$ the initial rodius. I the pinch current in amperes, $\rho_{0}$ the initial moss density, and $m$ the mass per unit length. For the following equations, all units will be cgs (with $I$ stil' in amps). Lefling $y=r / R_{0}$, and including the above expressions for $m$ and $B$, eq. (3.1) becomes.

$$
\frac{d}{d t}\left[\left(1-y^{2}\right) \frac{d y}{d t}\right]=-\frac{I^{2}}{\pi \rho_{0} 10^{2} R_{0}^{2}}\left(\frac{t}{y}\right) .
$$

The solution to this equation depends on the form of the current $I(f)$. Consider three different current profiles:

1. $I=s_{0} t$, where $s_{0}$ is a constant. A numerical solution of eq. (3.4) with a lineorly increosing current is found by Anderson, et al., who define a dimensionless parameter $T$ 


$$
\tau=\left(\frac{\pi 10^{2} \rho_{0} R_{0}^{4}}{s_{0}^{2}}\right)^{-1 / 4} 1
$$

With $y(0)=1$, and $d y(0) / d \tau=0$, the normalized radius $y$ is found ta reach one-eighth of its initial radius for $T=1.43$. The Anderson poper defines the pinch time $t_{p}$ as the time for $y$ to reach $1 / 8$, which was a commonly observed final radius for the snowmplow pinch. The choice of the final radius is somewhat arbitrary, but not critical. becouse of the high shecth velocity near the final stages of the collapse. Choosing $y=0$, for example, gives a value for the pinch time that is only $3 \%$ longer. Since the final value of the radius does not greatly affect the time ta pinch, the convention that the plasma has pinched when $y=1 / 8$ is adopted. Thus, the lime to pinch with o linearly increasing current is

$$
r_{\rho}^{l}=1.43\left(\frac{\pi 10^{2} \rho_{o} R_{o}^{4}}{s_{o}^{2}}\right)^{1 / 4}
$$

The superscript $l$ denotes a linearly increasing current. The pinch time then scoles as $\rho_{0}^{1 / 4}, s_{0}^{-1 / 2}$, and is linear in $R_{0}$.

2. $I=I_{0}$, where $I_{0}$ is a constant. For a current which rises to - constont value in a time much shorter than the pinch time, the pinch dynamics can be madeled by lefting the current $I$ in eq. (3.4) be o constonf $I_{0}$. Define a now dimensionless time $T$ 


$$
T=\left(\frac{I_{0}}{\left(\pi 10^{2} \rho_{0}\right)^{1 / 2} R_{0}^{2}}\right)+.
$$

Now write eq. (3.4) in terms of $\tau$, exponding the differentials.

$$
\left(1-y^{2}\right) \frac{d^{2} y}{d \tau^{2}}-2 y^{2}\left(\frac{d y}{d \tau}\right)^{2}+\frac{1}{y}=0
$$

An analytic solution to this differential equation is given by Kamke. ${ }^{11}$ who expresses the solution in terms of an integral. Eq. (3.8) then reduces to the following integral, which must be evaluafed numerically.

$$
\tau=\int_{y}^{1} \frac{\left(1-y^{2}\right) d y}{\sqrt{y^{2}-2 \ln y-1}}
$$

For a lower limit $y=1 / 8$, the dimensionless pinch time is $T=.873$. This resulf is verified by both a numerical integrafion of eq. (3.9). and a numerical solution to eq. (3.4) with a constant current. In dimensioned variables, the time to pinch is

$$
f_{p}^{c}=.873 \frac{\left(\pi 10^{2} p_{o}\right)^{1 / 2} R_{0}^{2}}{I_{0}}
$$

The superscript $c$ denotes a constant current. Thus, the time to pinch with n manetant murpant eralas as $n 1 / 2$ R 2 and $T-1$ 
3. $I=s_{0} t, t \leq t_{1}$, and $I=s_{1} t, t>t_{1}$. With the fused-bank arrangement, the pinch current was found to rise quickly, and then level off to ither a constant, or a slowly increasing current. If the rate of rise of the second slope is small, the time to pinch could scale either as in case (1), or case (2), depending on what fraction of the pinch time is spent in the inearly increasing current regime. With the current as an arbitrary function of time, it is not possible to write eq. (3.4) in terms of a dimensionless time, rather it is necessary to numerically solve the dimensioned equation. This was done with a $2^{\text {nd }}$ order Runge-Kutta scheme. ${ }^{12}$ The results of one particular run, with the slope $s$, equal to zero, are shown in $f i g .3 .5$.

Although an analytic scaling of the pinch time was not found for the current that rises to a maximum, and levels off, it was noted from the numerical solution to eq. (3.4) for the case $s_{1}=0$, that with o fixed maximum current $I_{0}$ and $s_{a}=I_{\alpha} / t_{1}$, the time to pinch $t_{p}$ raried opproximately as

$$
t_{p}=a+b t_{1} .
$$

The parameters $a$ and $b$ are constants, and $f$, is constrained to be less than or equal to the lime for the plosma to pinch on the linear portion of the current profile. Otherwise, the solution is given by eq. (3.6). For $t_{1}=0$, the pinch time is given by eq. (3.10), which requires that $a=t c$. The constant $b$ is determined by noting that $t_{p}=t$, as $t_{1}$ is 

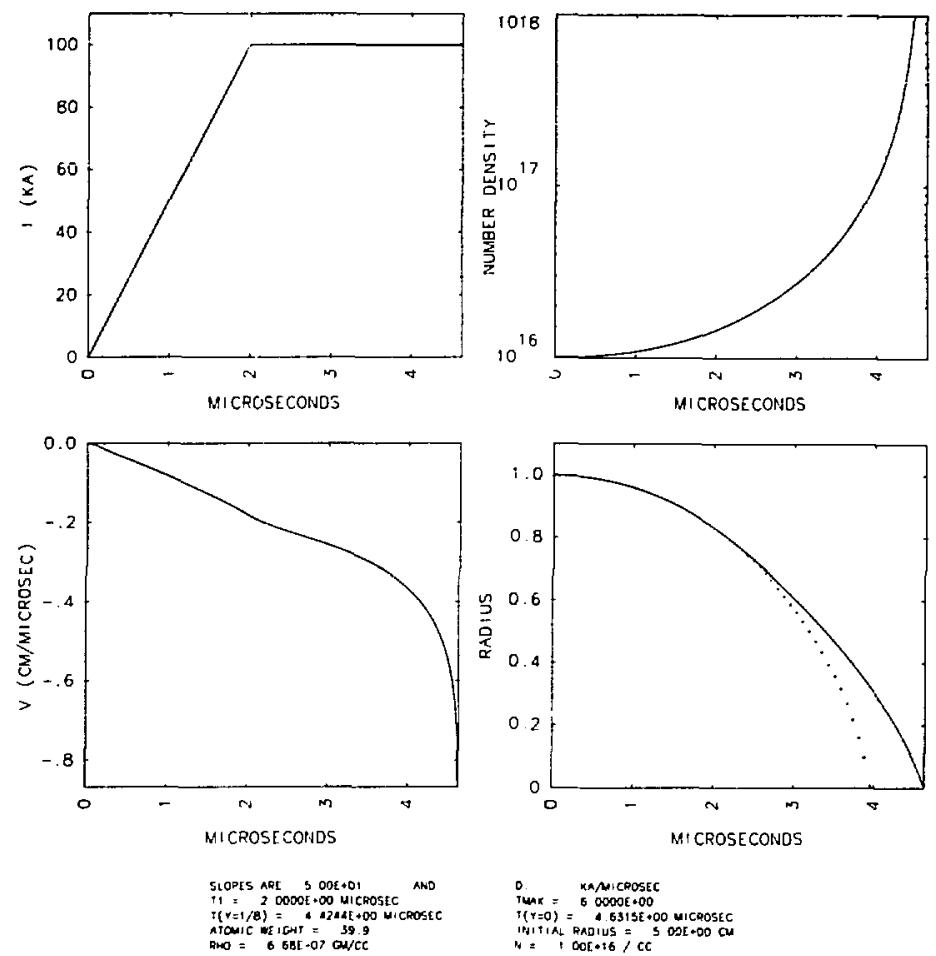

Figure 3.5. Numerical solution to eq. (3.4) with a current that rises to a maximum, and then levels-off to a constant. The dotted line is the solution if the current continues to rise linearly, rother thon leveling-off.

increased to the point where the current levels off just os the plasmo pinches. Using eq. (3.6) with $s=I_{0} / t_{1}$, the pinch time is 


$$
t_{p}=t_{1}=1.43\left(\frac{\pi 10^{2} \rho_{0} R_{0}{ }^{4}}{I_{0}{ }^{2}}\right)^{1 / 4} t_{1}^{1 / 2} .
$$

This equation is solved for $f_{1}$, and combined with eq. (3.10), noting that the various parameters cancel. Thus, $t_{l}$ is found to equal $2.34 f_{p}$ at this point, which makes $b=.573$. Writing out the coefficients, the pinch time is

$$
\begin{aligned}
f_{p} & =f_{p}^{c}+.573 t_{1}, t_{1} \leq 2.34 f_{p}^{c} \\
& =t_{p}^{l}, t_{1}>2.34 t_{p}^{c}
\end{aligned}
$$

where $t_{p}^{c}$ and $f_{p}^{l}$ are given by eqs. (3.10) and (3.6) respectively.

Note thot eq. (3.13) has been constructed to be equal to the solutions of cases (1) and (2) at either limit. For values of $t$, between the limits, the equation wos checked against the numerical solution and found to be about $3 \%$ low of mid-range, with a smaller error toward the boundaries. This error is well within those introduced by the approximations of the snow-plow model. A plot of the pinch time calculated by the snow-plow model versus $t$, for several runs, is shown in fig. 3.6, superimposed on a plof of eq. (3.13).

$$
\text { Upon writing out the expressions for } t_{p}^{c} \text { and } t_{p}^{l} \text {, eq. (3.13) }
$$
becomes 


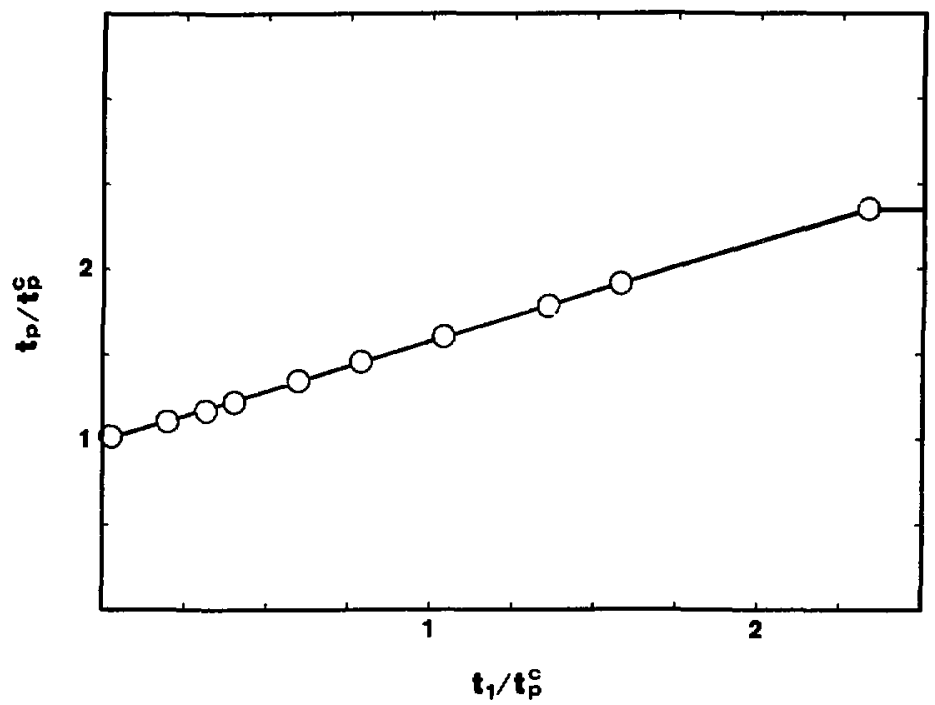

Figure 3.6. Pinch time versus the time the current levels off, in units of $t_{p}^{c}$. The circles are the numerical solutions to eq. (3.4), and the solid line is a plat of eq. (3.13).

$$
\begin{aligned}
t_{p} & =.873 \frac{\left(\pi 10^{2} \rho_{0}\right)^{1 / 2} R_{0}^{2}}{I_{0}}+.573 t_{1}, t \leq 2.04 \frac{\left(\pi 10^{2} \rho_{0}\right)^{1 / 2} R_{0}^{2}}{I_{0}} \\
& =1.43\left(\frac{\pi 10^{2} \rho_{0}}{s_{0}^{2}}\right)^{1 / 4}, t_{1}>2.04 \frac{\left(\pi 10^{2} \rho_{0}\right)^{1 / 2} R_{0}^{2}}{I_{0}}
\end{aligned}
$$


Perhaps one of the most interesting features of eq. (3.14) is that it is independent of the scaling of the linearly rising current solution, but rather depends. on the constant current scaling. Thus, the pinch time for these pinches would scale as $\rho_{0}{ }^{1 / 2}, R_{0}{ }^{2}$, and $I_{0}{ }^{-1}$, os does case (2).

For a fixed $R_{0}$ and $I_{0}$, the pinch current varies as

$$
t_{p}=a \rho_{0}^{1 / 2}+b \text {, }
$$

where $a$ and $b$ are constants equal to

$$
\begin{aligned}
& a=8.73 \frac{\sqrt{\pi} R_{0}{ }^{2}}{I_{0}} \\
& b=.573+t,
\end{aligned}
$$

which is exactly the scaling observed in $\mathrm{fig} .3 .4$.

The main difficulty encountered in applying sq. (3.15) is that the initial radius $R_{0}$ is not known, except by estimates from the initial gas distribution, and by accounting for the time delay to the bank fire, assuming the gas jet radius expands at the sound velocity. The moss density is determined by using the plot in fig. 2.5, the estimoted radius, and the known pinch length. Estimates of this sort were done with several shots, and the calculated pinch times were found to be within $10 \%$ of the measured values. 
The pinch time is also experimentally observed to vory linearly

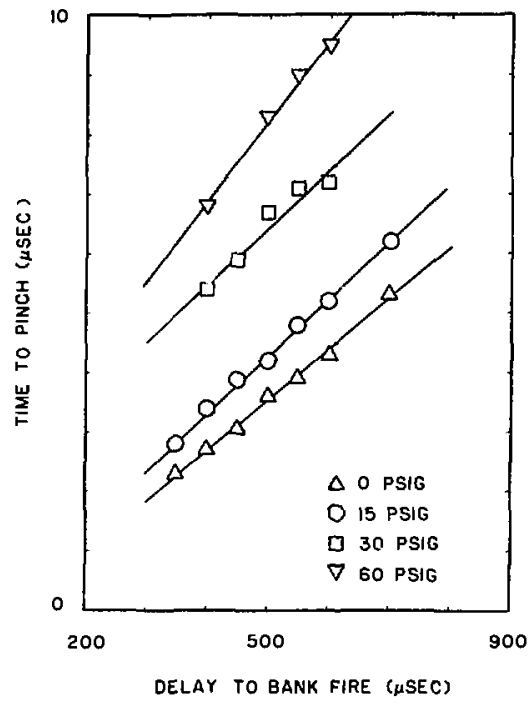

Figure 3.7. Measured time to pinch versus bank fire delay time (time in $\mu \mathrm{sec})$. for several plenum fill pressures.

with the bank-fire delay time $t$, as is seen in fig. 3.7. The radius $R_{0}$ of the gas puff is proportional to $t$, and, since the total gas quantity between the electrodes remains constant at later times (sea fig. 2.5), the density $\rho_{0}$ vories os $R_{0}^{-2}$, or $t^{-2}$. Thus, $t_{p} \propto \rho_{0}{ }^{1 / 2} R_{0}{ }^{2} \propto f$, from eq. (3.14), which is consistent with observations. 
Two aspects of the axial variation of the pinch sheuld olso be discussed. First, the froming camera photographs show that the pinch collapses to the axis first near the cathode, and last at the anode. If the current flows mostly in the skin, as is indicated by the interferogram in fig. 3.12 and by the light emission from the fan-shaped regions in the framing camera photagraphs, ther tr 3 pinch velacity is not entirely radial but has an axial component directed toward the anode. The magnitude of the axial velocity is ther the sine of the angle $\alpha$ which the fan-shaped region makes the z-axis just frior to peak coripression, times the maximum pinch velocity. Fo: $\alpha$ equal to $25^{\circ}$, which is the angle observed in fig. 3.8 of the next section, the axial velocity is 0.4 times the pinch velocity. Second, jince the mass density and gas jef radius vary with the axial posit on $z$, the pinch time is also expected to vary with $z$. From sec. 3.1.1 the density was found to fall off as $z^{-1}$, and the radius to be approximately linear with $z$. Thus, the time to pinch scales as $z^{3 / 4}$ for the scaling eq. (3.6), and as $z^{3 / 2}$ for eq. (3.10). The excerimentally observed scaling of the pinch time in $z$ was found to vary irom shot to shot, but was generally of the form $f_{p}(z) \propto a+b z$, where a and $b$ are constants.

\subsection{Examples of Pinch Formation and Lifetimes}

Two modes of initializing linear z-pinches were used: (1) the fused bonk, no gun mode, and (2) the plasma gun initiated mode. Both 
show oxample shots from each of these types, discussing the parameter variations. These included use of low and high $Z$ goses, short and long pinch lengths (23 mm vs $53 \mathrm{~mm}$ ), and use of various densities. In each of the shots studied, the pinch was observed to have an instability which eventually destroyed the pinch. These instabilities are examined

Table 3.1. Description of the of shots shown in this chapter

\begin{tabular}{lccc}
\hline Figure & Description & $\begin{array}{c}\text { Gength Plasma Fused } \\
(\mathrm{mm})\end{array}$ & $\begin{array}{c}\text { Gun } \\
\end{array}$
\end{tabular}

$3.8,3.9$ low density gas-puff, short

3.10 low density gas-puff, long

Ar 31 $\mathbf{x}$

3.11 high density gas-puff, long

Ar 53

$x$

3.12 high density, high $Z$, long

Ar 53

$x$

$\mathrm{Kr} \quad 53$

$\mathrm{He} \quad 53$

3.13 low $Z$ gas-puff, long

$\mathrm{N}_{2} \quad 53$

3.14 low $Z$, high current, long

3.15 plasmo gun initiated, short

$\mathrm{D}_{2} \quad 23$

in chapter 4.

\subsubsection{The gas-puff initiated Z-pinch}

Framing camera photographs of a gas-puff initiated z-pinch are shown in tigs. 3.8 and 3.9. The photograph is consistent with the diagram of the chamber in fig. 2.3. I That is, the cathode is on the right and the cnode on the left. This convention is also used with all 
Table 3.2. Measured parameters for the shots shown as examples in this section. These are the values measured at peak compression.

\begin{tabular}{lcccccc}
\hline \hline Figure & $\begin{array}{c}\text { Radius } \\
(\min )\end{array}$ & $\begin{array}{l}V_{\text {bonk }} \\
(\mathrm{kV})\end{array}$ & $\begin{array}{c}I_{\text {pinch }} \\
(\mathrm{kA})\end{array}$ & $\begin{array}{c}\tau_{\text {conf }} \\
(\mu \mathrm{s})\end{array}$ & $\begin{array}{c}\tau_{\text {pinch }} \\
(\mu \mathrm{s})\end{array}$ & $\begin{array}{c}\pi_{\varepsilon} \\
\left(\mathrm{cm}^{-3}\right)\end{array}$ \\
\hline 3.8 .3 .9 & 2.0 & 4.0 & 100 & 0.6 & 1.3 & $1.5 \times 10^{18}$ \\
3.10 & 2.0 & 4.0 & 90 & 1.0 & 2.0 & $5 \times 10^{19 b}$ \\
3.11 & 3.5 & 5.5 & 210 & 0.6 & 7.5 & $3 \times 10^{19 b}$ \\
3.12 & 2.0 & 4.0 & 120 & 0.5 & 6.0 & $2 \times 10^{19}$ \\
3.13 & 2.7 & 4.0 & 140 & $<0.1$ & 3.5 & $1.3 \times 10^{18}$ \\
3.14 & 2.7 & 5.5 & 220 & 0.4 & 6.5 & $2 \times 10^{19}$ \\
3.15 & 1.0 & $8.0^{\circ}$ & 120 & 0.2 & 3.0 & $6 \times 10^{19}$
\end{tabular}

a Although the voltage was higher for these shots, the barik energy was lower since a $112 \mu \mathrm{F}$ capocitor bank was used. The other shots were done with the $900 \mu \mathrm{F}$ copacitor bank.

bThese values are estimates based on gas valve settings, and the Saha equation.

other framing camera photographs. Niote also that fig. 3.8 , as well as others in this chapter, is constructed of a sequence of several shots. varying only the camera timing. A comparison of the parameters af these shots with the others in this section is given tables $3.1,3.2$. and 3.3 . In table 3.1 the fypes of shots observed are listed. Table 3.2 gives a comparison of the directly measured parameters from these 

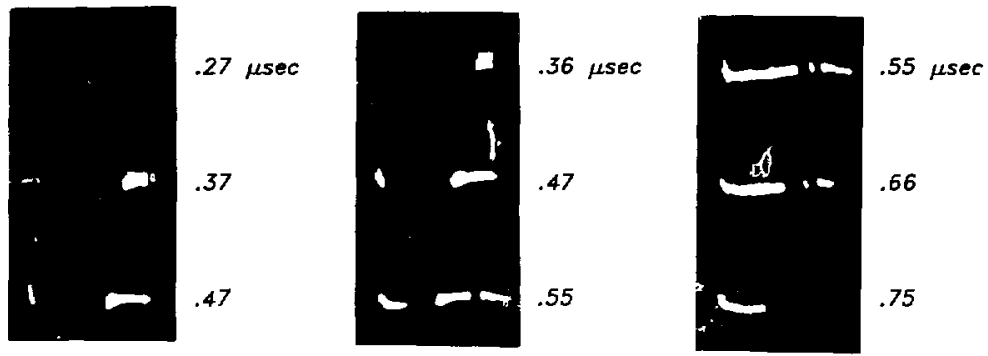

Figure 3.8. Typical life history of a lower density argon Z-pinch from framing camera photographs. The time in usec from the pinch current start is given to the right of each frame. The electrode separation is $31 \mathrm{~mm}$.

shots, and table 3.3 compares the deduced parameters. Figure 3.8 shows the typical evolution of the gas puff, fused-bank operation. Argon was used instead of deuterium to make the pinch eosier to photograph. The gas comes out of the plasma gun orifice in the form of a conically-shoped jet, is ionized, and starts to pinch, as shown in the first frame. (For orientation, note the shodow of the tip of the gun electrode.) Subsequent frames show that the conically-shaped plasma then pinches to a straight calumn, starting from the cathode end. It then breaks up in a sausage-like instability. Other shots of the same configuration, but with different densities, show the same pinching characteristics, but different break-up mechanisms. Some pinches are 


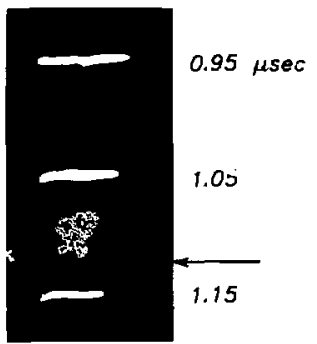

(a)
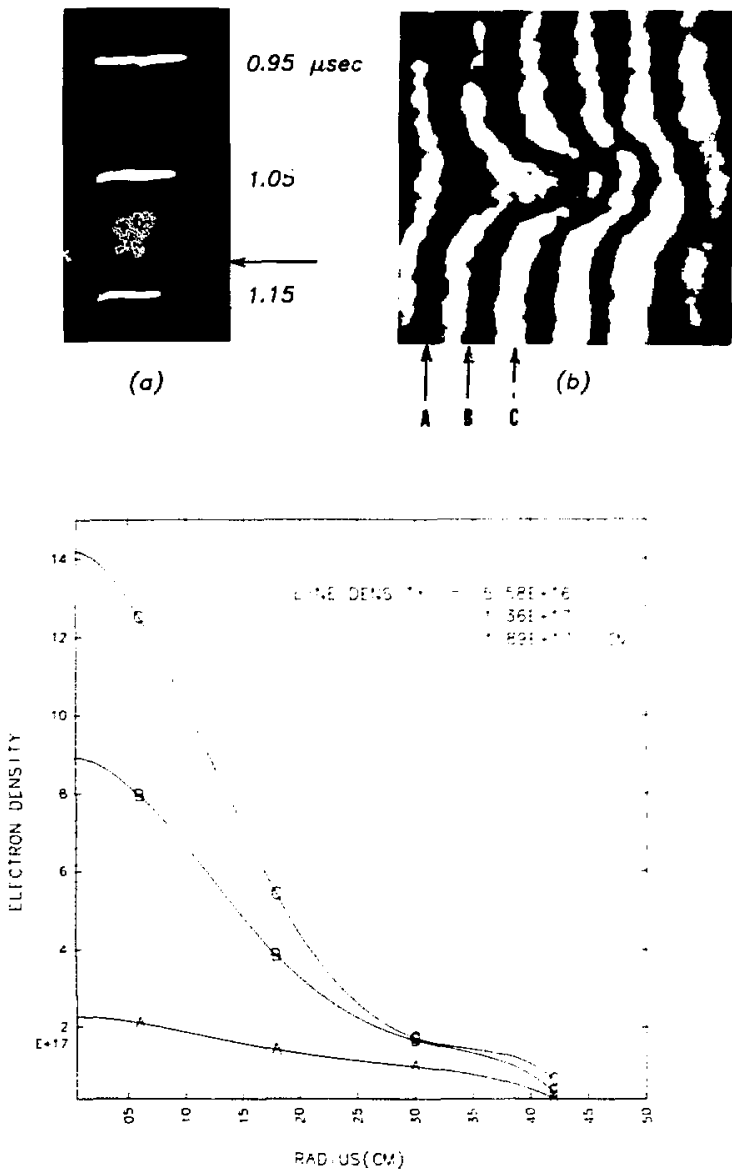

(c)

Figure 3.9. Density measurement for the type of pinch shown in fig. 3.8. (a) Framing camera photographs, with a pinch length of $31 \mathrm{~mm}$. (b) Interferogram from a Mach-Zehnder inferferometer operated at $632.8 \mathrm{~nm}$. The position of the arrow represents the relative time of exposure in $\mu s e c$. (c) Abel inversions at the indicated axial oosifions. 
observed to remain as a straight column up to $0.6 \mu \mathrm{sec}$. The observed instability scaling with density is discussed in chapter 4, however.

Fig. 3.9 shows the electron density measurement for a gas-puff initiated z-pinch. The Abel inversion of the cross-sections in (b) are given in (c), where the position of the letters on the plots represent the calculated densities as a function of $r$. The solid lines are a least-squares fit of the points to a lhird order polynamial with a zero slope of the pinch axis. The line density is found to vary from $6 \times 10^{16}$ to $1.9 \times 10^{17} \mathrm{~cm}^{-1}$ along the axis of the pinch. This density implies a Bennett 13 temperature of 200 to $490 \mathrm{eV}$ along the axis. Other deduced parameters are disrussed in the next section.

Fig. 3.10 shows the evolution of a gas-puff initiated, fused-bank pinch with the electrode separation increased to $53 \mathrm{~mm}$. There are no direct measurements of the electron density for this set of shots, but the electron line density is estimated from the gas valve settings, from the results of appendix $A$, and the ianization calculations of oppendix $\mathrm{C}$ to be $6 \times 10^{18} \mathrm{~cm}^{-:}$. The technique by which this is done is explained in sec. 3.3 .

Fig. $3.10(b)$ shows the current and voltage versus $t$ for the shot. The current is observed to start with the large voltage spike shown on the lower trace. This voltage is measured ot the capacitor bank side of the spark gap, and shows the inductive effect of the fuse blowing. As the current across the gap and the pinch rises, the voltage quickly drans. and ramains low until the oinch-column is disruated. Tha encond 


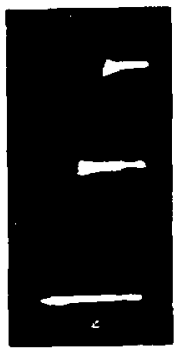

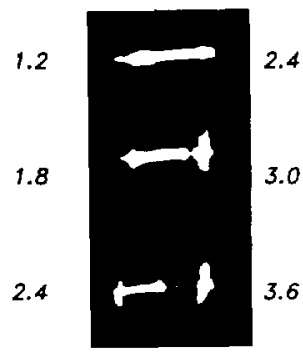

(a)

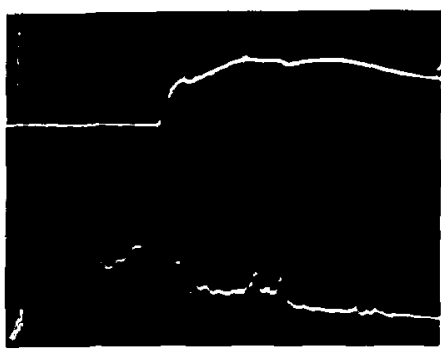

(b)

Figure 3.10. Evolution of argon, gas-puff initiated Z-pinch with an electrade separafion of $53 \mathrm{~mm}$. (a) Framing camera photagraphs of two shots differing only in camero timing. The time in usec from pinch initiation is shown to the right of each frame. (b) The upper trace is the pinch current af $100 \mathrm{kA} /$ div., and the lower trace is the anode volfage af $4.0 \mathrm{kV} / \mathrm{div}$. The horizontal scale is 5.0 $\mu$ sec/div.

voltage spike occurs of this time, and the current is abserved to dip, but does not go to zero, because of the inductance of the system. Thergafter, a pinch often reforms, but usually with a lawer inductance path. These restrikes were not studied extensively, however.

Hord $x$-roy signals were also observed to coincide with the voltage spikes. For those shots which had several restrikes and breaks, additional x-ray bursts were observed which also coincided with the later inductive voltage spikes. The energy of these bursts wos 
measured by absorption methods, and the results are discussed in the

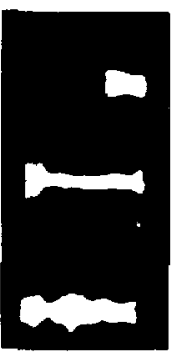

(a)

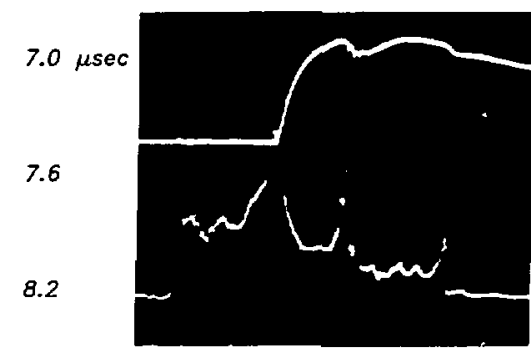

(b)

Figure 3.11. Evolution of a long, higher density orgon Z-pinch. (a) Framing camera photograph of one shot with an electrode separation of $53 \mathrm{~mm}$. Time shown in usec from pinch current start. (b) The upper trace is the pinch current, $100 \mathrm{kA} /$ div., and the lower troce is the onode voltage, $4.0 \mathrm{kV} / \mathrm{div}$. The horizontal scale is $5 \mu \mathrm{sec} / \mathrm{div}$.

next chapter.

Figure 3.11 shows an example of a long, high density argon pinch, for which the pinch current of peok compression is $210 \mathrm{kA}$. Density measurements were not obtained for this and similior shots because of the excessive light from the plasma. Estimates from the gos valve settings indicated on ion density of $3 \times 10^{15} \mathrm{~cm}^{-3}$. These estimates ore made by use of $\mathrm{fig}$. 2.5, using the plenum fill pressure and the bank fire timing to abtain the totol quantity of gas puffed into the chamber. The line density is estimated by dividing this quantity by the pinch length, and the locol density is estimated by assuming the 


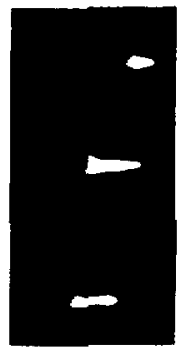

(a)
$4.4 \mu \mathrm{sec}$

5.0

5.5

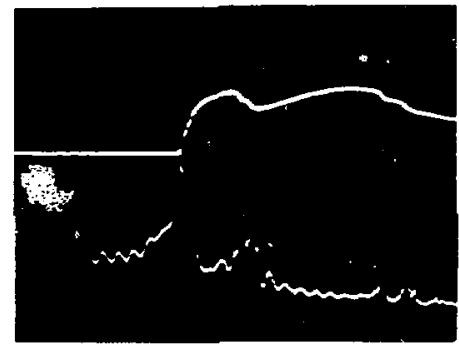

(b)

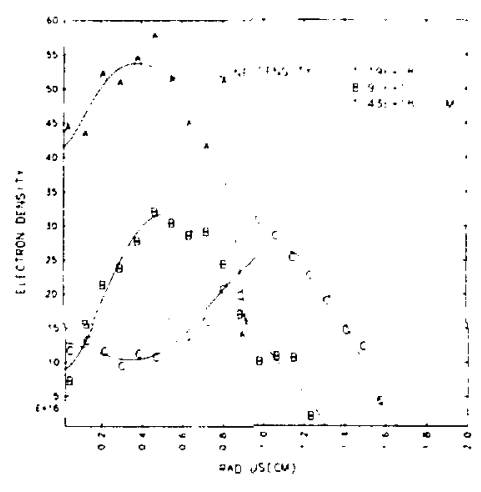

(d)

Figure 3.12. Evolution of a krypton pinch. (o) framing camera photograph. (b) The upper trace is the pinch current, $100 \mathrm{kA} /$ div., and the lower troce is the anode voltage, $4.0 \mathrm{kV} / \mathrm{div}$. The horizontal scale is $5 \mu \mathrm{sec}$. (c) Interferogram during pinch formation. (d) Abel inversion of the density profile. 
camero image. Use of the Bennett relation, and the ionization calculations indicate a temperature of only $3 \mathrm{eV}$.

Figure 3.12 shows an example of krypton shot, with an interferogram at the early stages of pinching, and its Abel inversion. The measured electron density is $1.2 \times 10^{18} \mathrm{~cm}^{-1}$, whereas using the gas valve colculations the ion density is estimated to be $5 \times 10^{17} \mathrm{~cm}^{-1}$. The difference between the two could very wall be aftributed to the plasmo being doubly ionized. From these measurements, the electron density at peak compression is estimoted to be $2 \times 10^{19} \mathrm{~cm}^{-1}$. It was not possible to get density meosurements af peak compression, presumably because of the higher femperafure, which leads to a higher ionization state, resulting in more electrons, and a much higher fringe shift.

Another inferesting feature of this class of shots is that during the compression the region of highest density is at the edge of the plasmu. This is seen clearly in the interferogram and Abel inversion, and olso in the framing camera photographs. In the darker regions of the pinch the optical emission is strongest near the edge, and weaker in the center. An abel inversion of the light, which is also proportionol to the density for line radiation, would also show much higher densities at the edge. This type of density profile is very similiar to the infinitesmally thin plasma shell that is considered with the snow-plow model. 


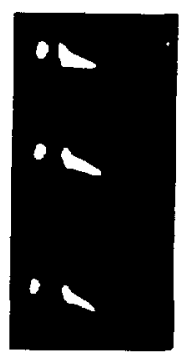

(a)

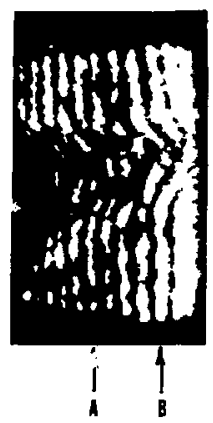

(c)
$6.8 \mu \mathrm{sec}$

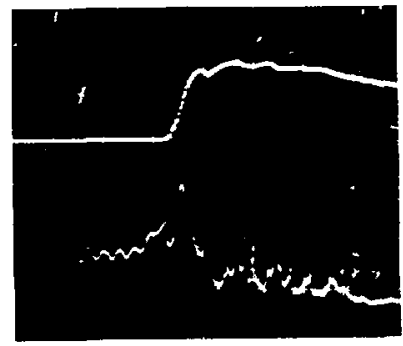

(b)

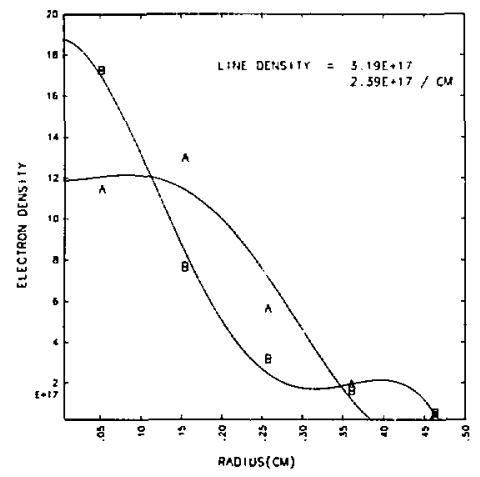

(d)

Figure 3.13. Example of a helium Z-pinch. (o) Froming camera photographs with the time of the frome given in usec. (b) The upper trace is pinch current, 100 $\mathrm{kA} /$ div., and the lower trace is the anode voltage, $4.0 \mathrm{kV} / \mathrm{div}$. The horizontal scale is $5 \mu \mathrm{sec} /$ div. (c) Interferogram at $7.0 \mu \mathrm{sec}$. (d) Abel inversions at the 
Fig. 3.13 shaws an example of a helium pinch. The measured electron density is $1.3 \times 10^{18} \mathrm{~cm}^{-3}$. The time of the frames shown is after the first pinch and break-up, so that the plasmo seen is likely a restrike, and contains electrode material. The helium and hydrogen pinches were very difficult to obtain, and often came aport on ane end before the other end could pinch.

Figure 3.14, the last gas-puff example, is of a nitrogen pinch. The ion line density, determined from the gas valve settings, ond by comparing with electron density measurements of similar shots, is

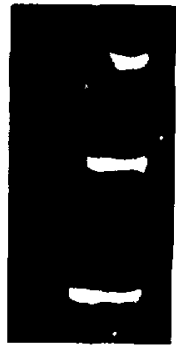

(a)

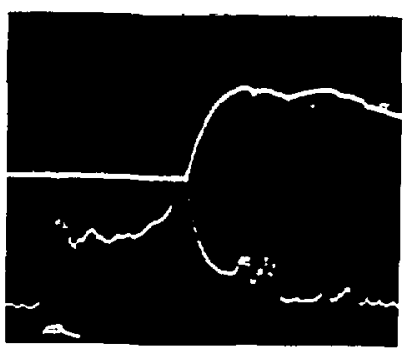

(b)

Figure 3.14. Example of a nitrogen pinch. (a) Framing camera photograph. (b) Pinch current, $100 \mathrm{kA} / d i v$. , and anode voltage, $4.0 \mathrm{kV} /$ div. The horizontal scalo is 5 usec/div.

$1 \times 10^{18} \mathrm{~cm}^{-1}$. 
3.2.2. The plasina gun initicted Z-pinch

An example of a deuterium, plasma-gun inifiated z-pinch is shown
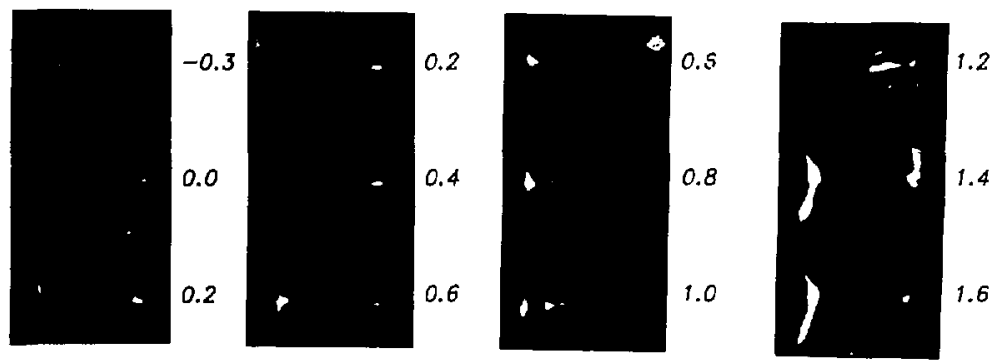

Figure 3.15. Evolution of a deflagration gun initiated deuferium Z-pinch, assembled from framing camera photographs of four shots with only the camero timing varying between shots. Time in $\mu$ sec from start of pinch current is given to the right of each frame. The electrode seoaration is $23 \mathrm{~mm}$.

in fig. 3.15. For this set of shots, the onode had a conically-shaped protrusion, the outline of which is best seen in the frames of 0.0 and $1.6 \mu \mathrm{sec}$. This configuration was used to insure that the pinch remained on the central axis to enable phatographing. I. was later found that the pinch could also be prevented fram wandering by dishing the anode by $4^{\circ}$ toward the center.

The first frame of fig. 3.15 shows the plasma as it is shot from the deflagration gun. At $t=0.0 \mu \mathrm{sec}$ the pinch current begins. At $t=0.6 \mu \mathrm{sec}$ the current is still rising olmost linearly, and has 
reached approximately $80 \mathrm{kA}$. A $t=0.6 \mu$ sec a straight pinch channel is seen, which then develops a dark region near the anade at $t \sim 0.8 \mu$ ser The dark region then propagates axially to the opposite olectrode $u$ at $1.4 \mu \mathrm{sec}$ no light is seen at all. The frames at 1.4 and $1.6 \mu=\doteq c$ show that anode has become much brighter after the pinch light goas out. The light pipe/photomultiplier combination also shows the light to increase almost a factor of ten at this time. One possible explanation is that high-z electrode material is being ionized at ihese later times, greatly increasing the line radiation.

No electron density measurements were made with the gun-initiated shots, therefore it is not possibie to reliably use the Bennett relation to estimate electron and ion temperatures. However, from the framing camera photographs in fig. 3.15, it is possible to estimate the ion density by observing the velocity of the propagating dark region in the pholographs. Any disruption of the magnetic field must flow at the Alfven velocity, which is a function of the magnetic field strength and the ion density. Equating the observed velocity to the Alfuen velocity, and using $B=260 \mathrm{kG}$ (obtained from the current and pinch radius), the ion density is found to be $6 \times 10^{18} \mathrm{~cm}^{-3}$. The electron line density is $1.9 \times 10^{17}$, which means that $s=11$, by eq. (1.5). Based on this density, and considering the pinch to be fully-ionized, the sennett temperature is found to be $120 \mathrm{eV}$.

The tims the column remains straight without breaking apart, is estimoted from the frames at 0.6 and $0.8 \mu \mathrm{sac}$ to be less than $200 \mathrm{nsec}$; 
perhops only 100 nsec. This gives a respectable nt of $4 \times 10^{12}$. (The ion temperature is much too low to consider fusion, however.)

The FLR scaling $s$ is determined to be 22 by using eq. (i.5) and the estimated electron line density.

\subsection{Deduced Parameters}

In table 3.3 several deduced parameters are shown for the example shots shown in the last section. How these parameters were determined is discussed in the following poragraphs.

The electron density $n_{\theta}$ and the electron line density $N_{\theta}$ $\left(=\pi n_{a} a^{2}\right)$ were most reliably determined from loser interferometry and subsequent Abel inversion. Then by using the Bennett relation and the results of the average ion charge calculations, it is possible to determine the average ion charge $Z_{a v e}$, and the temperature $T\left(T_{0}=T_{i}\right)$ by an iterative scheme. The Bennett relation is

$$
I^{2}=200 N_{0}\left(1+\frac{1}{Z_{o v \theta}}\right) k T \text {. }
$$

where the units are $I$ in amperes, $N_{0}$ in $\mathrm{cm}^{-1}$, and $k T$ in ergs. A value of $Z_{\text {ore }}$ is guessed, and then eq. (3.17) is solved for the temperature. This temperature is then used with the plots in appendix $C$ to find a 
$Z_{\text {ave }} N_{i}$. It was often found that the inferred ion temperature was too high for a consistent result, however. Therefore, many of the daduced parameters for these shots can only be estimates.

So far, it has been assumed that $T_{e}=T_{i}$. The equilibration between electrons and ions is discussed by Spitzer, 14 who shows the time behavior of the temperature as

$$
\frac{d T_{i}}{d t}=\frac{T_{a}-T_{i}}{T_{\theta q}}
$$

The quontity $\tau_{e q}$ is the equipartition time, and is defined as

$$
\tau_{\theta q}=\frac{3 m m_{f} k^{3 / 2}}{8(2 \pi)^{1 / 2} n_{f} Z^{2} Z_{f}^{2} e^{4} \ln \Lambda}\left(\frac{T}{m}+\frac{T_{f}}{M_{f}}\right)^{3 / 2}
$$

where the subscript $f$ is used to differentiate between field and test particles. For ions heating in a field of electrons eq. (3.19) becomes

$$
\tau_{e q}=3.18 \times 178 \frac{A T_{b}^{3 / 2}}{2 n_{0} \ln A} \text {, }
$$

where $T_{0}$ is in electron volts and $n_{b}$ is in $\mathrm{cm}^{-3}$, and $A=m_{i} / m_{p}$. The Coulomb logarithm term InA is given by Book ${ }^{15}$ as 


$$
\begin{aligned}
\ln \Lambda & =23-\ln \left(\frac{n_{0}^{i / 2} Z}{T_{0}^{3 / 2}}\right), T_{0} \leq 10 \mathrm{eV} \\
& =24-\ln \left(\frac{n_{0}^{1 / 2}}{T_{0}}\right), T_{0}>10 \mathrm{eV}
\end{aligned}
$$

The value of the FLR porameter $s$ is determined by eqs.(1.5) and (1.6). For fully ionized plasmas, $s$ varies os $Z^{1 / 2}$ for higher $z$ plasmas. However, if the plasma has a low enough temperature that it is only singly ionized, then scales as $A^{-1 / 2}$, the inverse square root of the mass ratio. A related parameter, the overage number of ion orbits in the pinch diameter $\left.a /<a_{i}\right\rangle$, is taken as $s / \pi$, as is discussed in chapter 1. The scaling of the plasma stability as a function of $s$ is presenied in the next chapter.

The quantity $v_{d e} / v_{t i}$, the ratio of the electron drift velocity to the ion thermal velocity, is determined by using eq. (1.8). If this ratio becomes $\geq 1$. the plasma becomes subject to micro-instabilities.

3.4. Electron density profiles

Electron density profile meosurements were made of a number of shots by doing Abel inversions of the interferograms, and $f i t+i n g$ the points to curves using a least squares fit. These profiles are shown in appendix D. It is found that at peak compression the profiles fit 
best to a Bennett profile, rather than a gaussian or quadratic profile. All thrce of these profiles are discribed in the appendix.

References for chapter 3

${ }^{1}$ A. H. Shapiro, The Dynamics and Thermodynamiss of Compressisie Fluid flow, (Ronald, New York, 1953), Vol. 1, pp. 74-78.

2J. K. Vennard, Elementary Fluid Mechanics, $4^{\text {th }}$ ed. (Wiley. New York, 1961), pp. 156-158.

3P. L. Gwen, and C. K. Thornhill, Aer. Res. Council Reports and Memoranda No. 2616, Great Britian, 1948.

4F. S. Sherman, in Rarefied Gas Dynamics, edited by J. A. Laurmann (Academic, New York, 1963), Vol. 2, pp. 250-258.

5E. S. Love, C. E. Grigsby, L. P. Lee, and M. J. Woodling, NASA Technical Report R-6, 1959.

6H. Ashkenas, and F. S. Sherman, in Rarefied Gas Dynamics, edited by J. H. de Leeuw (Academic. New York, 1965), Vol. 2, p. 84.

${ }^{7}$ G. A. Bird, in Rarefied Gas Dynamics, edited by K. Karameheti (Acodemic. New York, 1974), p. 207. 
D. T. A. Blair, in Electrical Breakdown of Gases, edited by J. M. Keek and J. D. Craggs (Wi ley, New York, 1978), PP. 558-562.

${ }^{9} 0$. A. Anderson, W. R. Baker, S. A. Colgate, J. Isle, Jr., and R. V. Pyle, Phys. Rev. 110, 1375 (1958).

${ }^{10} \mathrm{~S}$. Glasstone, and R. H. Loveberg, in Cantrolled Thermonuclear Reactions (Van Nostrand, Princeton, 1960), pp. 230-234.

$1^{1} \mathrm{E}$. Kamke, Differentialgleichungen, LBsungsmethoden und Lbsungen. 6 th ed. (Akodemische, Leipzig, 1959), p. 594, para. 6.224.

12 Handbook of Mathematical Functions, edited by M. Abramowitz and I. A. Stegun (Dover, New York, 1964), p. 897, para. 25.5.17.

13w. H. Bennet1. Phys. Rev., 45, 890(1934).

${ }^{14}$ L. Spitzer. Jr.. Physics of Full, Tonized Goses, $2^{\text {nd }}$ es. (iviley, New York, 1962). PP. 135-136.

15D. L, Book, "NRL Plasma Formulary". NRL Memorandum Report No. 3332, p. 12 . 
CHAPTER 4

\author{
The Pinch Break-up \\ and \\ Observed Instabilities
}

The intent of this chapter is to describe the phenomeno, and present the measurements related to the break-up of the z-pinch, leaving the analysis of these observations ond measurements to the next chapter. In section 4.1 the break-tup is choracterized, and examples of the most common instabilities shown. In section 4.2 the measured parameters ore given. Section 4.2 .1 contains the $x$-ray energy measurements. In section 4.2 .2 the observed break-up velocities, and linear growth rates are given. Finally, in section 4.2 .3 the scaling 
of the instability type with the flR parameter $s$ is presented, which is probably one of the most interesting results of the experiment.

4. 1 Description of the break-up processes

\subsubsection{Scenario}

The formation of the pinch is amply described and shown in the last chapter. However, it was also apparent, especially in figures 3.8 and 3.10 , that the initially straight pinch column is abruptly broken. Shortly before the time of the disruption, a dip in the pinch current, and an anode voltage spike are observed. The magnitude of the voltage spike is often two to three times the bank charge voltage. It is always seen, and the plasma column may or may not exhibit a disruption at that time. In figs. 3.8 and 3.10 a disruption associated with the voltage spike is seen. Fig. 4.1 (a) shows, on the other hand, a pinch which remains for more than $1 \mu$ sec after the voltage spike, which coincides with the first frame. It is also interesting to note that the sausoge-like instabilities of the first frame have been obliterated by the noxt frame.

The half-width of the voltage spike was observed to be $\sim 100 \mathrm{nsec}$ far those pinches which had only one break. For those with multiple breaks, a more jagged, and longer lasting voltage spike was seen. 


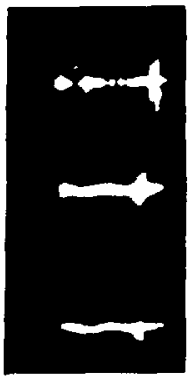

4.7

4.1
$3.4 \mu \mathrm{sec}$

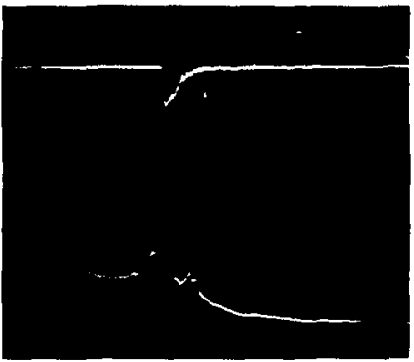

(b)

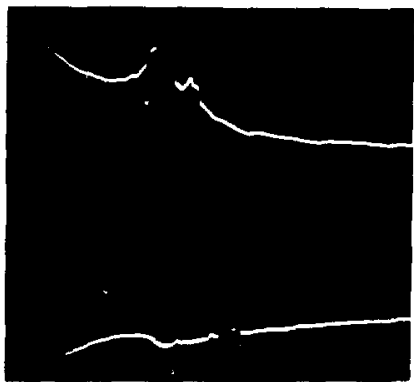

(d)

Figure 4.1. (a) Framing camero photographs (b) $X$-ray signal and anode voltoge, $2 \mathrm{kA} / \mathrm{div}$. The time scale is $5 \mu \mathrm{sec} / \mathrm{div}$.

(c) pinch current, $100 \mathrm{kA} /$ div., and anode voltoge, $2 \mathrm{kA} / \mathrm{div}$. The time scale is $5 \mu \mathrm{sec} / \mathrm{div}$. (d) Anode voltoge ond pinch current, same scales, but at $1 \mu \mathrm{sec} / \mathrm{div}$. The three pulses on the pinch current trace show the timing of the camero fromes. 
contributed to the anode voltage. Often, the plasma was observed to form sausage like islands of light and dark intensities, much like those seen starting to torm in tig. 4.2 .

Also observed, and coinciding with the anode voltage spike, was o hard $x$-ray signal. Figure 4.1(b) shows the x-ray signal from a NaI (TI) crystal-photomultiplier combination. The leading edge of the x-ray burst coincides with the voltage spike, to within the resolution of the oscilloscope troces. The decay time of the pulse is due to the slow decay of the detector. Later shots using a plastic scintillator show that the $x$-ray pulse duration is approximately equal to the voltage spike width. The energy of the $x$-roys, meosured by absorption methods, is found to be $\sim 10 \mathrm{keV}$, whereas the height of the voltage spike was about $11 \mathrm{kV}$. These measurements oro give in section 4.2 .1 . The source of the $x$-rays was not established, since attempts to obtoin $x$-roy pinhole photographs were not successful.

The eventual break-up of the pinch was very dependent on the initial parameters, i.e., the bonk-fire time, and the pulsed gos valve fill pressure and stop odjustments. With some pinches, a sausage like discuption would develop before the entire length of the pinch was formed. With others, the pinch could be observed to remain straight for more that $1 \mu \mathrm{sec}$ before either breaking up, or slowly expanding. The vorious type of instabilities which were observed to couse these disruptions are discussed in the next section. 
Events occurring after the pinch broke up were not studied extensively. The pinch current remained high, with the current presumably finding alternative paths. However, on many accasions the plasma was observed to repinch, and a second, and sometimes third X-ray burst were seen. Damage marks indlcate that much of the later current was from the anode edge to the wall, thus shorting-out the cathode. Furthermore, the framing camera was seldom able to pick up images at these late times, even though the total light was much higher.

\subsubsection{Types of pinch instabilities}

By far the most commonly observed deformation was the sausage-like, or $m=0$ mode instability. Clear examples of these have already been seen in figs. 3.8 and 4.1 . Other examples, which show an abrupt disruption which propagates down the length of the pirch, also appear to be sausage-like. An example of this type is seen in fig. 3.10. In figure 4.2 another such example is shown, where close examination of the framing camera image in (a) shows brightness variations possibly corresponding to a sousage-like structure. However, when the pinch does break up, it does not break up into droplets, but rather comes apart between two of the "links" of the sausage. The link-sausage structure is seen more clearly in the contour plots in (b).

The location of the break is most often seen to originate at the 


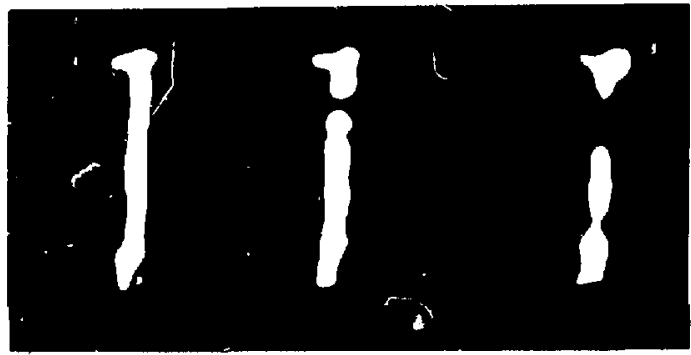

(a)

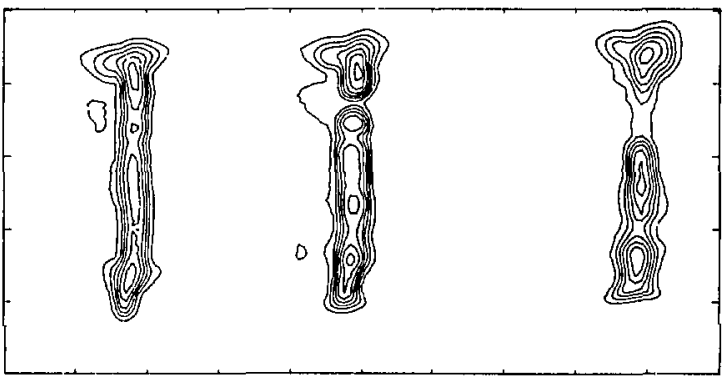

(b)

Figure 4.2. (a) Framing camero photograph of a shot with a sausage-like disruption. (b) Contour plot of a photo-densitometer scun of the framing camera image. The electrode gap is $53 \mathrm{~mm}$, and the frame separation $200 \mathrm{nsec}$. The cathode is on the top, and time goes from left to right.

shots, the reverse is also seen, although it is not as common. Interferograms of the dark region between the sausages also show that the electron density is much less than that of the brighter regians of 
region, or it is rapidly heated and thus exponds. Often, an apparently lower density filament appears to remain in the darker region. The anly evidence for the core region is from the framing camera photogrophs, for which the imoge intensity is proportional to the square of the electron density. The interferograms do not show this effect. Examples of shots showing the propagating expansion region, or

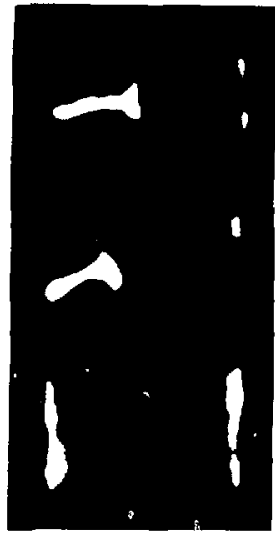

(a)

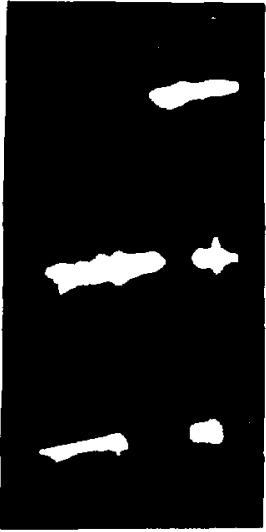

(b)

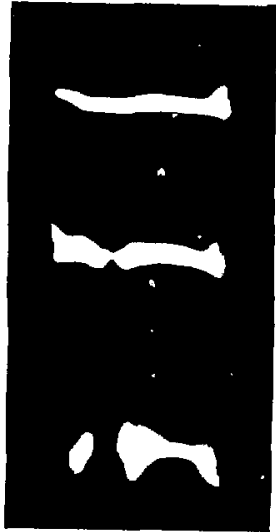

(c)

Figure 4.3. Framing camera photographs of sausage-like instabilities which propagate across the pinch. (a) The instability can be seen as on expanding expansion plume, in this shot. (b) A faint, lower density core appears to have formed in the lower density region of the pinch. (c) The pinch appears to be rapidly expanding in a radial direction. 
Another instability thut is seen with the higher line densi shots is the long wave-length kink, or $m=1$ mode. This instabili results in gross motions which distort the pinch column, causing it eventually break oport. Examples of two shots with the kink mode a

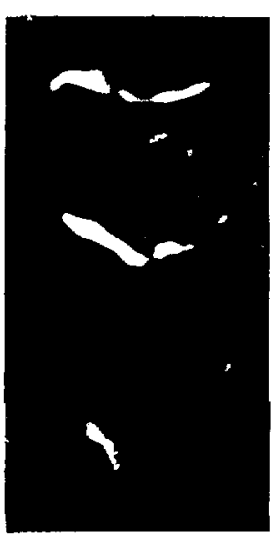

(a)

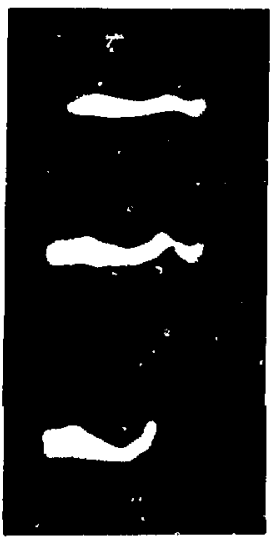

(b)

Figure 4.4. Two higher density shots showing the development of the kil instability. The time between frames is $200 \mathrm{nsec}$, and the electrode gap is $53 \mathrm{n}$ for both shots.

shown in fig. 4.4. Higher order modes ( $m=1$ ) have not been observed. 
At very low densities a very wispy, short-lived pinch is observed. Framing camera photographs show that the pinch breaks apart near the cathode befare the plasma nearer the anode can pinch. Then later, the plasma near the anode pinches, but that nearer the cathode has exponded to a largo, dark plume. At the other end of the scale, i.e., with high density, fat and cool pinches, the pinch column undergoes a non-uniform radial expansion, with the pinch looking much like o fat, tuzzy caterpillar. This fuzziness, or spindle structure as it is sometimes called, has been observed in many previous experiments. and is considered to be the non-linear MHD $m=0$ mode. ${ }^{1}$ Examples of both of these types of pinches are shown in fig. 4.5 .

\subsubsection{Other features}

Two other interesting features were noted related to the instabilities. The first is that often, in the case of pinches whica. remain straight after the voltage spike, an oscillation on the anocie voltoge was observed. The oscillation is seen in fig. 4.6, which is the anode voltage trace for such a shot. This oscillation in the anode voltage was not seen with all the shots, however. Since the current is only slowly varying in this time scale, the voltage variation must be due to rapid variations of the pinch inductance. The inductance is proportional to $\ln (R / a)$, where $R$ is the chamber radius. Thus if appears that the pinch is oscillating about a small radius. 


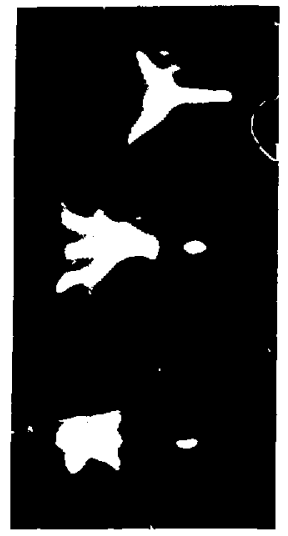

(o)

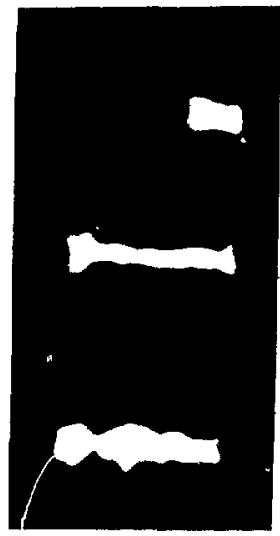

(b)

Figure 4.5. Framing comero photographs of (o) low density, wispy pinch, whict pinches and blows apart on one side before it can pinch on the other, and (b) - bigin line density pinch which exponds radially, and non-uniformily along its length. The frome spacing is $500 \mathrm{nsec}$, and the electrode gap $53 \mathrm{~mm}$ for bott shots.

The other interesting feature was observed when the pinch chamber was disassembled. The anode, which is copper, was found to have a $6 \mathrm{~mm}$ diameler and $2 \mathrm{~mm}$ deep spherical depression, which was dug by the gimat Tt wae lanatod rirently in the center of the anode. on the 


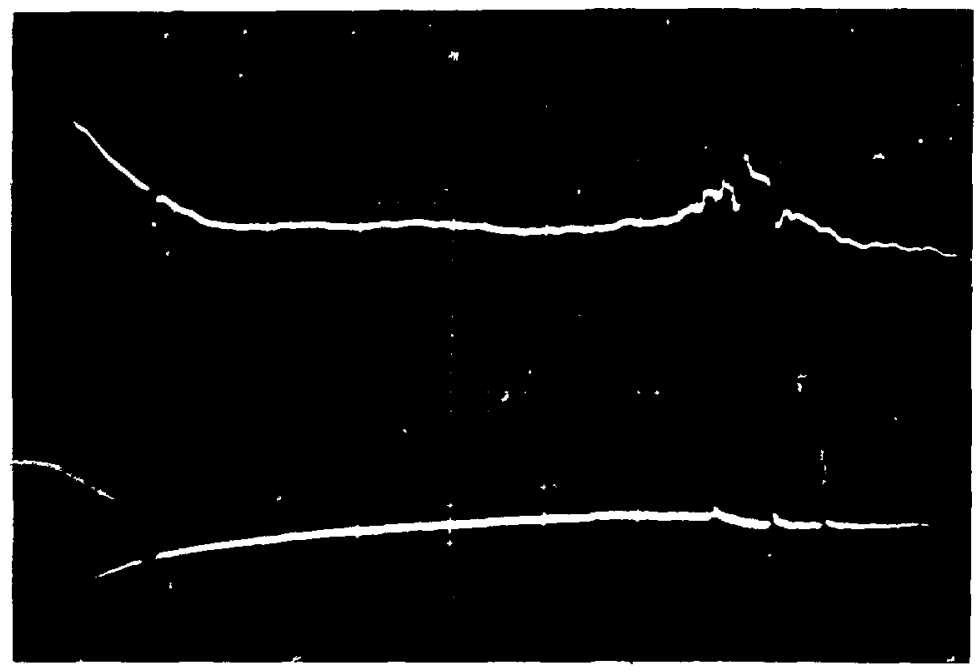

Figure 4.6. Anode voltage, $4 \mathrm{kV} / \mathrm{div}$, and pinch current, $100 \mathrm{kA} / \mathrm{div}$. for a shot where the pinch radius oscillates, producing aperiodic variation in the anode voltage just prior to the voltage spike. The horizontal scale is $1.0 \mu \mathrm{sec} / \mathrm{div}$.

chamber axis. This anode was used for neorly 400 shots, with pinch curretts common!y ranging from 100 to $200 \mathrm{kA}$, and up to over $500 \mathrm{kA}$ with a few of the later shots. The cathode, on the other hand, showed no such damage. 


\subsection{Measured parameters}

\subsubsection{X-ray absorption measurements}

The energy of the burst of x-rays associated with the voltage spike was measured by on $x$-ray absorption technique, as described in chapter 2. Aluminum foils of varying thickness were placed in front of the SPM defector, and the maximum intensity per shot measured. Fig. 4.7 shows such plots for three separate configurations of the pinch and detector. Neutral density filters were placed between the scintillator and the photomultiplier to keep the detector from saturating. The data points shown in the figure are averages over several shots. With those points where the detector was near saturation rejected. The last point on curve $B$ is probably too high because of the stroy light getting into the defector, and therefore was not used in the determination of the $x$-ray energy.

The mass attenuation coefficient $\mu / \rho$ was determined from fig. 4.7 by the technique discussed in stupter 2, and was used to determine the x-ray energy with the plot given by Osher. 2 These results ore summarized in table 4.1. It is interesting to note that the x-ray energy is much greater than the plasma tempe-oture, and corresponds closely to the anode spike valtage $v_{s}$. 


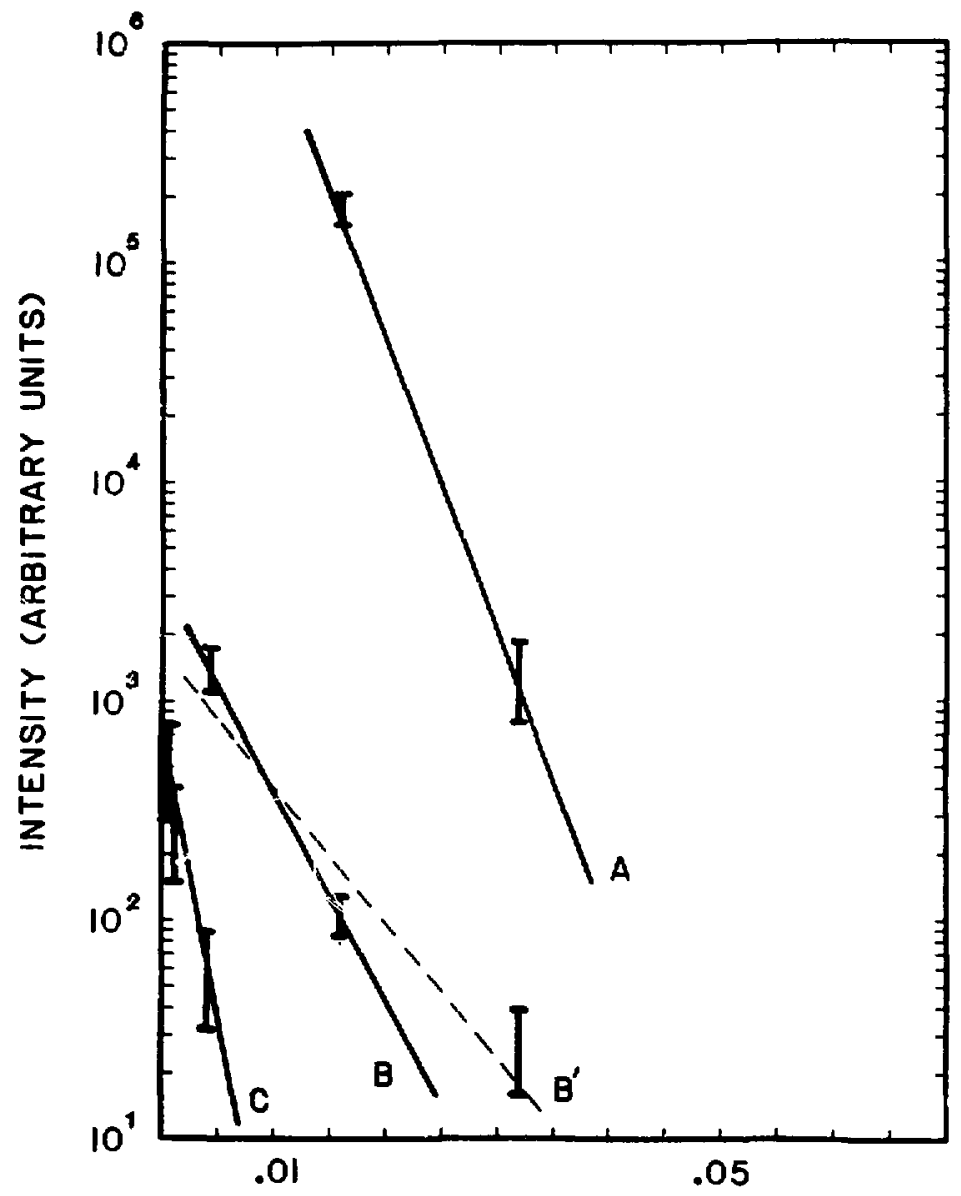

ABSORBER THICKNESS (IN.)

Figure 4.7. X-ray intensity versus Al toil thickness, for three separate configurations of the pinch and SPM detector. The iines connecting points are a least squares fit for those curvas with more 
gnt $2 i$ aly ytitnoup ont . T.A grueit mort otob to yrommuz .1.t oldot

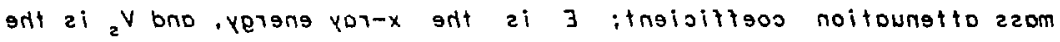
. sxiqe sbono sitt to egotioy

\begin{tabular}{|c|c|c|c|}
\hline $\begin{array}{c}e^{V} \\
(V x)\end{array}$ & $\begin{array}{c}3 \\
(V \ominus \lambda)\end{array}$ & $\begin{array}{c}q \backslash 4 \\
\left(e \backslash S_{m o}\right)\end{array}$ & osneLpez \\
\hline$i \pm e$ & $z . \neq 0.8$ & $A \pm A B$ & $A$ \\
\hline $1 \pm 11$ & z. $\pm 8 . e$ & $a \pm 1 \varepsilon$ & $\theta$ \\
\hline $1 \pm 8$ & $5 . \pm 8.2$ & $O S=8 e$ & 3 \\
\hline
\end{tabular}

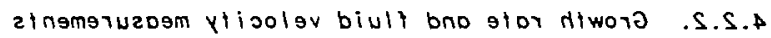

ton grgw zotor htworg rognil, ytilidoteni exil-ggozude ont ntiw

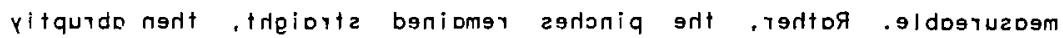

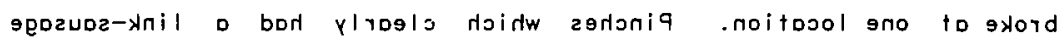

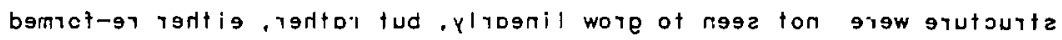

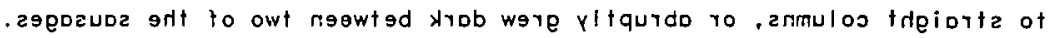
svont theim zebom $0=m$ besiilidota-elitorg yltmeroqgo bmo , bevigado ent

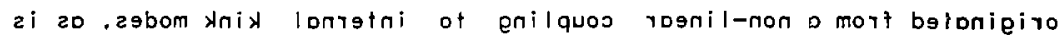

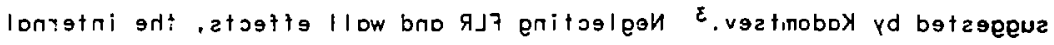

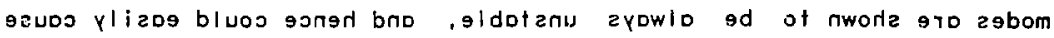
. TOSqqo of zebom ierto 
The axial velocity of the break-up of the pinch column is found to range from 8 to $8 \times 10^{6} \mathrm{~cm} / \mathrm{sec}$ for the argon pinches with a $4 \mathrm{kV}$ bank charge voltage. The velocities are mildly density dependent, with the lowest densities hoving the largest break-up velocities. These velocities are an order of magnitude greater than the axial component of the snowplow sheet during the pinch compression. Hence, it does not appear that they are due simply to plasma streaming. They are of the same order of magnitude as the Alfven velocity, however, where $v_{A}$ is determined using densities from the gas valve output calculations.

The radial expansion of the plasma in the dark region was very quick, and difficult to determine because as the plasmo expands the light emission decreases to the point that it is no longer visible. However, lower limits on the expansion velocity con be made from framing camera photographs of the break-up. These indicate that the lower bound on the radial velocity at the break is about $i \times 10^{7} \mathrm{~cm} / \mathrm{sec}$. This velocity is then somewhat larger than the Alfven velocity, suggesting that some other mechanism is the source of the rapid expansions.

Kink instabilities were not generally observed, except for those shots where the density was high enough. When they were observed, the growth rotes were found to be linear. Thus, it seems sensible to characterize them in terms of dimensionless parameters used in the linearized WHD theory. Thus consider $k a$, the wave number $(k=2 \pi / \lambda)$ times the binch rodius. and ro/y.. the measured arowth rote. times $a$ 
and divided by the Alfuon volacity. The Alfuen velocity is calculated by using the magnetic field of the plosmo edge, and using the gas valve

Table 4.2. Measured growth raies for the kink $(m=1)$ instability. The first three quantities are measured from the framing camera photographs. The Alfven velocity is calculated at the plasma radius, and by using the density determinad from the graph in fig. 2.5.

\begin{tabular}{|c|c|c|c|c|c|}
\hline Shot no. & $\begin{array}{c}k \\
\left(\mathrm{~cm}^{-1}\right)\end{array}$ & ko & $\left(10^{6} \sec ^{-1}\right)$ & $\left(10^{V_{A}} \mathrm{~cm} / \mathrm{sec}\right)$ & $\mathrm{ra} / \mathrm{V}_{A}$ \\
\hline 108 & 9.5 & 2.9 & 1.2 & 4.0 & .009 \\
\hline 292 & 4.6 & 1.4 & 1.6 & 1.3 & .1 \\
\hline 296 & 1.2 & .36 & 2.2 & 1.3 & .04 \\
\hline 303 & .59 & .18 & 2.4 & 1.3 & .05 \\
\hline 593 & 1.8 & .54 & 2.4 & 4.6 & .3 \\
\hline 672 & 3.5 & 1.0 & 4.4 & 3.9 & .06 \\
\hline
\end{tabular}

dynamics to defermine the mass density. These measurements are compared with the theoretical values in the next chopter.

4.2.3. Instability type scaling with the FLR parameter.

A quick scan through the shots reveals that the type of instability, and the length of time the pinch remains straight, is very dependent on the amount of gas puffed into the chamber, which is proportional to the line density. Vary low density shots, as is shown 
densities more typical sausage insiabilities cre observed. ivith yet higher gas densities, straight pinches winch last several microseconds are seen. At higher densities, the classical kink instabilities are seen, and at the highest gas densities studied, fat, low temperature pinches are observed to expand in a radial direction, but at different velocities along the axis.

Since these effects are also dependent on the temperature, it is important to use a scaling which includes both temperature and density,

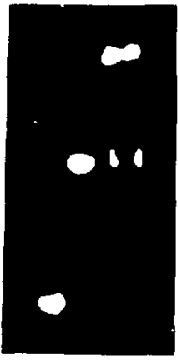

$A$

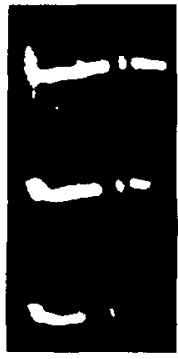

$B$

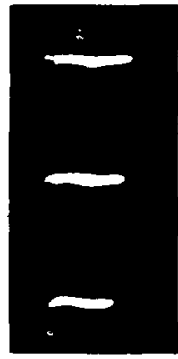

C

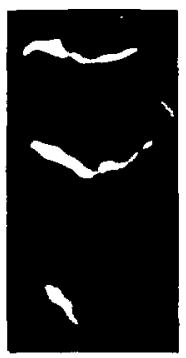

$D$

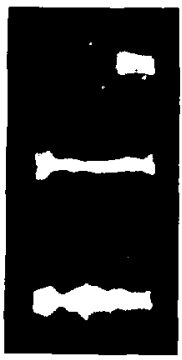

$\varepsilon$

Figure 4.8. A comparison of the types of instabilities observed, arranged in ascending order of the quantity of neutral gas puffed into the chamber. Refer to table 4.3 for the parameters.

as well as the average ion chorge. This suggests scaling the instability types with the FLR parameter $s$, as is defined in eq. (1.5), which is related to the strength of the FLR corrections to the MHD 
Table 4.3. Description and paramelers for the pinch instabilities seen in fig. 4.8.

\begin{tabular}{|c|c|c|c|c|c|}
\hline & Description Ior & $\begin{array}{l}\text { Ilne density } \\
\left(\mathrm{cm}^{-1}\right)\end{array}$ & $z_{\text {ov }}$ & $s$ & $a /\langle a\rangle$, \\
\hline$A$ & $\begin{array}{l}\text { wispy, does not } \\
\text { completely pinch }\end{array}$ & $3 \times 10^{16}$ & 8 & 8.2 & 2.6 \\
\hline $\mathrm{B}$ & sausage like & $5 \times 10^{16}$ & 12 & 16 & 5 \\
\hline C & $\begin{array}{l}\text { straight, slowly } \\
\text { expands }\end{array}$ & $2 \times 10^{17} a$ & 14 & 45 & 14 \\
\hline D & kink & $4 \times 10^{18}$ & 2 & 27 & 9 \\
\hline$E$ & non-linear sausage & $1 \times 10^{19}$ & 3 & 47 & 15 \\
\hline
\end{tabular}

a This value comes from the measured electron density, using the Bennett relation to obtain the temperature, which is used to find $Z_{a v}$, and hence the line density. The gas valve dynamics give the value $1 \times 10^{18}$.

theory. These results are shown in fig. 4.8, which shows the variation of the instability type versus $s$.

Unfortunately, density measurements were only made with a few of these types. Therefore it is not possible to accurately know the line densities of all of these pinches. The primary means of determing these values thus rests on the PGV dynamics, which at best cre only very approximate calculations. But, in spite of the uncertainity of 
these numbers, the general scaling of the instabilities is very obvious.

References for chapter 4

1D. L. Book and M. Lampe, NRL Memorandum Report 3334 (1976).

${ }^{2}$ See reference 20 , chapter 2 .

${ }^{3}$ B. B. Kadomtser, in Reviews of Plasma Physics, edited by M. A. Leontovich (Consultants, New York, 1966), vol. 2, p. 168. 


\title{
CHAPTER 5
}

\author{
Discussion
}

In this chapter the results of the measurements and observations are discussed. Section 5.1 contains a discussion of the growth rate colculations, using both the skin current and diffuse current models. Finite orbit effects on the growth rates ore then discussed, and measured and calculated growth rates are compared. In section 5.2 possible causes for the voltage spike and $x$-ray burst are discussed. These include enhanced resistivity effects, inductive effects of filamentation, radiation collapse, and electromagnetic effects. 


\subsection{Linear growth rate comparisons}

Comparison of experimentally observed growth rates with those predicted by the linearized, ideal MHD theory is very risky, primarily for two reasons. (1) The experimentally observed defurmations are generally large anplitude, thereby violating the initial premise of the linearized theory that the perturbations are small. Thus, to make a realible comparison, one would have to use growth rates predicted by a non-linear theory. (2) It is found from the linearized theary that the predicted growth rates are strongly dependent on which equilibrium profiles in pressure and magnetic field are used.3,13 Therefore, i! is necessary to know both the pressure and current variation with $r$ to provide a valid comporison with even the linear theory.

In spite of these difficulties, it would be useful to know what growth rates could be expectad from the linearized, MHD theory, both with and without the FLR damping effects, and with arbitrary equilibrium profiles. A comparison could then be mode with experimentally observed growth rates, provided they are small amplitude, and it is checked that the growths are not saturated; i.e., that the perturbed quantities grow as exp $(\gamma t)$. Furthermore, equilibrium profiles, which are needed for the theory, could be determined from the abel inversions of the interferometer data. It is 
thereby hoped that some conclusions on the magnitude of the FLR effects might be made.

Therefore, to prepare for this comparison, the next several paragraphs are devoted to a discussion of the relevant MHD theory. Since a non-linear theary does not exist, the discussion is limited to small amplitude (linear) calculations.

\subsubsection{Analytical techniques}

Calculations which determine the growth rates of an unstable, equilibrium plasma are usually based on procedures formally established in a paper by Bernstein, ef al.1 The approach is to first consider small variations from an equilibrium solution to the ideal (meaning infinite conductivity), magnetohydrodynamic (MHD) equations, keeping only first order terms in the variation. The variations of the various parameters are written in terms of a vector $\xi$, which is a measure of the displacement of the location of a fluid element from its equilibrium position. Thus, the location $r$ of a fluid element at time $t$ is

$$
\mathbf{r}=r_{0}+\boldsymbol{\xi} .
$$

The ideal MHD equations are thus written in the form 


$$
\rho_{0} \frac{\partial^{2} \xi}{\partial t^{2}}=\boldsymbol{F}(\xi)
$$

where

$$
\begin{aligned}
\boldsymbol{F}(\xi)= & \nabla_{0}\left[\gamma p_{0} \nabla \cdot \xi+\left(\xi \cdot \nabla_{0}\right) p_{0}\right]+\frac{1}{\mu_{0}}\left[\boldsymbol{I}_{0} \times \boldsymbol{a}-\boldsymbol{\Delta}_{0} \times\left(\nabla_{0} \times \boldsymbol{0}\right)\right] \\
& +\left[\nabla_{0} \cdot\left(\mu_{0} \xi\right)\right] \nabla_{0} \Phi_{0} .
\end{aligned}
$$

The subscripts on the operators imply that they operate on the zeroeth order quantities only. The vector 0 is the first order variation of the magnetic field and equols

$$
\boldsymbol{0}=\nabla_{0} \times\left(\xi \times \boldsymbol{\omega}_{0}\right)
$$

Note that the form of $\boldsymbol{F}$ depends on which equilibrium, and which coordinate system is used.

All the first order quantities are now written as

$$
Q(r, \theta, z, t)=Q(r) \exp (i \omega t+i m \theta+i k z)
$$

and eq. (5.1) becomes the eigenvalue equation 


$$
-\omega^{2} \rho_{0} \xi=\boldsymbol{F}(\xi, \omega) .
$$

The associated boundary conditions are that $r \xi_{F}=0$ at $r=0$, and $\xi_{r}=0$ at $r=b$, the location of the wall, it the plasma extends to the wall. This boundary condition restricts the solution to the internal modes of the pinch, since the plasma boundary is then fixed to the wall. For a pinch surrounded by a vacuum region, the boundary conditions are more complicated. The first requirement is that the zeroeth and first order pressures be continucus across plasma-vacuum inferface. This results in the following two conditions. ${ }^{2}$

$$
p_{0}+\frac{B_{0 i}^{2}}{2 \mu_{0}}=\frac{B_{00}^{2}}{2 \mu_{0}}
$$

and,

$$
-\gamma p_{0} \nabla \cdot \xi+\frac{1}{\mu_{0}} \boldsymbol{E}_{0 ;} \cdot \boldsymbol{E}_{i}=\frac{1}{\mu_{0}} \boldsymbol{\omega}_{00} \cdot \boldsymbol{\theta}_{1}+\frac{\xi_{r}}{2 \mu_{0}}\left(\frac{\partial B_{0 e}{ }^{2}}{\partial r}-\frac{\partial B_{0 i}{ }^{2}}{\partial r}\right) .
$$

One further boundary condition, which is a result of the requirement that the tangential component of the electric field be continuous across the boundary, is that 


$$
\cdot \boldsymbol{\theta}_{10}=\boldsymbol{n} \cdot\left[\nabla \times\left(\xi \times \boldsymbol{\theta}_{0.0}\right)\right]
$$

Note, that in eqs. (5.5)-(5.7), the subscripts 0 and $t$ refer to the zeroeth and first order quantities, respectively, and the subscripts $i$ and e refer to the quantities interior and exterior to the plasma. The explicit form of $9 q .(5.4)$ is too long to write out here, but can be found in a paper by Goedbloed and Hagebeuk. 3 There it is written in cylindrical coordinates, includes a longitudinal magnetic field, and allows arbitrary profiles for the zero order pressure and magnetic field, provided they satisfy the equilibrium condition

$$
\nabla\left(p+\frac{\boldsymbol{D}^{2}}{2 \mu_{0}}\right)=\frac{(\boldsymbol{a} \cdot \nabla) \boldsymbol{\theta}}{\mu_{c}} .
$$

Eq. (5.4) has been solved for the growth rates for various configurations by several early researchers.4-11 Kruskal and Schworzschild solved for $m=0$ growth rates of a linear pinch with a skin current, having no $B_{z}$ nor wall-stabilization. Taylers extended the model to a diffuse current, uniform density pinch, for which the pressure varies as $p=p_{0}\left(q-(r / a)^{2}\right)$. For this particular equilibrium eq. (5.4) can be onalytically reduced to a transcendental equation in terms of the boundory values, from which the maximum growth rate can be obtained. These results are discussed in a followinn suhsention 
Tayler later axtended the work to include wall affects and a stabilizing $B_{z}{ }^{6}$ These efforts were paralleled by several Russian researchers. Trubnikov ${ }^{7}$ considered a uniform current distribution, and no axial field, to find approximate solutions to $m=0$ growth rates for a limited numbar of wavelengths. Shafranov added wall and axial magnetic field effects to a skin current model, and later to a uniform densily, diffuse current model, 9 doing an analysis very similar to that of Tayler. A good review of the growth rate calculations, and general stability principles is given in an article by Kadomtsev. 10

The diffuse current form of eq. (5.4) was first written in general form by Hain and LUst.' 't who numerically calculates $m=1$ and higher mode growth rates using a Bennett profile. Their analysis includes on axial magnetic field, and considers a vacuum region outside the plasma which extends to infinily. Their calculations, however. only consider $k 0$ in the range 0.1 to 1.0 . and with $B_{z} \neq 0$. Their results are therefore not suited for the present work. After the poper on the diffuse pinch stability theory by Newcomb ${ }^{2}$ growth rote calculations of diffuse pinches stopped. The subject was however reopened ten years later by Friedberg's to determine the effects on the growth rate of diffuse versus skin current distributions. His analysis is done with an incompressible $(\Gamma \cdot \xi=0)$ appraximation, with tokamak ordering $\left(B_{z} \gg B_{\theta}\right)$, and with the plasma extending to the wall. Therefore, the results do not apply to the present experiment, but they do show a strong dependence on the current profile, with the growth 
the diffuse profiles. Goedbloed and Hagebeuk 3 extended the analysis by considering the plasma to be compressible, but they also kept the same ordering in $B_{Y} / B_{\theta}$.

Thus, it is apparent, that to compare experimentally measured growth rates with diffuse growth rate calculations for anything but a uniform density, it is necessary to first numerically calculate the growth rates from eq. (5.4), using the appropriate boundary conditions. This will not be done, however. Rather, the approach will be to campare observed growth rates with those predicted by the uniform particle current density model of Tayler. And os is also done in his paper, estimates of the variation of the growth rates with non-uniform particle density profiles will be made.

\section{1.2. Skin current model growth rates}

In order to understand the general features of the growth rates, consider first a skin current model. Growth rates calculated by this model, with $B_{z}=0$, are given by Kadomtsev. 14

$$
\frac{\omega^{2} a^{2}}{v_{A}^{2}}=-k a \frac{I_{m}^{\prime}(k a)}{I_{m}(k a)}\left(m^{2} X_{m}(k a, k b)+1\right)
$$

Here $k$ is the wavenumber, $a$ is the pinch radius, $b$ is the woll radius, $v_{A}$ is the Alfuen velocity, and $I_{m}$ is the modified Bessel function of the first kind, and of order $m$. Similiarly, $\mathcal{K}_{m}$ is a function of Bessel 


$$
K_{m}(k a, k b)=\frac{K_{m}(k a) k b I_{m}^{\prime}(k b)+I_{m}(k a) k b K_{m}^{\prime}(k b)}{k a K_{m}^{\prime}(k a) k b I_{m}^{\prime}(k b)+k a I_{m}^{\prime}(k a) k b K_{m}^{\prime}(k b)} .
$$

For large wall radil, eq. (5.10) becomes

$$
\mathcal{K}_{m}(k a, k b)=\frac{K_{m}(k a)}{k a K_{m}(k a)}, \text { for } b \gg a .
$$

And for large values of $k a, K_{m} \approx 1$.

The growth rate is defined as $\gamma= \pm i \omega$. Normalized to $\gamma_{4} / 0$. it is plotted as a function of ka in fig. (5.1) for $m=0$, and $m=1$ modes. Several features are readily apparent. (1) The larger growth rates occur for the smallest wavelengths, (2) the wall has no stabilizing effect on the $m=0$ mode, and (3) the wall effects are stronger for long wavelengths and negligible for sinort wavelengths. Plots far higher order modes show that the growth rate is much higher for long wavelength:, despite the stronger wall stabilization. Therefore, from the skin-current model, with no $B_{z}$, it appears that short wavelength MHD instabilities will dominate for $m=0$ and $m=1$. and that higher order modes are also unstable.

\subsubsection{Diffuse current model growth rates.}

If the current is not restricted to flow on the surface of the pinch, the predicted growth rates vary substantially from those

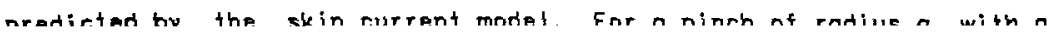


SKIN CURRENT WODEL, $M=0$

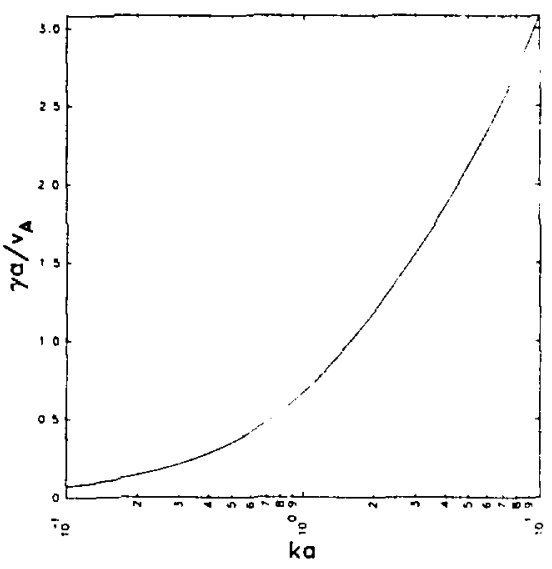

(a)

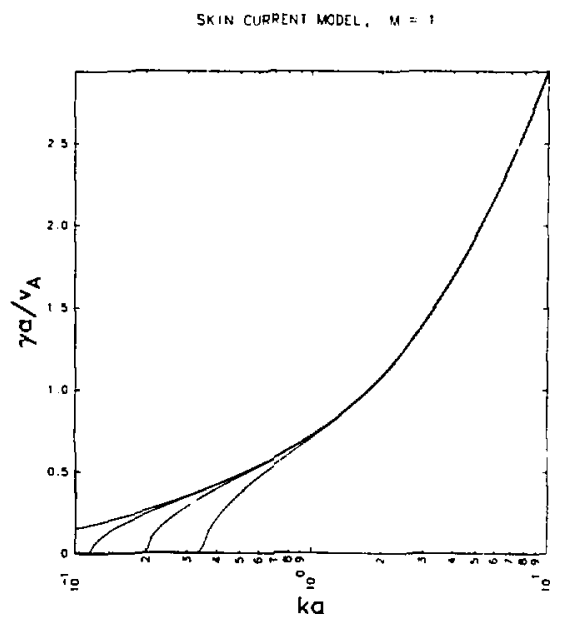

(b)

Figure (5.1). Grawth rate versus ka for (a) $m=0$, and (b) $m=1$, for a wall radius to pinch radius ratio of $5,10,20$, and 50 . 
uniform density $\rho_{0}$, and a pressure $\rho_{0}\left(1-(r / a)^{2}\right)$, an equilibrium is obtained with a uniform current density $J_{0}$, and a magnetic field $B_{\theta}(r)=B_{\theta 0} r j a$, with $p_{0}=B_{\theta 0}{ }^{2} / \mu_{0}$. With this equilibrium eq. (5.4) can be reduced to Bessel's equation, with on incompressible fluid opproximation. By applying the boundary conditions a transcendental equation for the normalized growth rate $\Omega^{2}\left(\Omega^{2}=\gamma^{2} a^{2} / v_{A}{ }^{2}\right)$ can be found. This is given by Tayler ${ }^{5}$ as

$$
\left(\frac{\varphi}{x}\right)_{r=0}=-\frac{m^{2}+\Omega^{2}}{m^{2}+\Omega^{2} \rho_{1} / \rho_{0}} k 0 \frac{K_{m}^{\prime}(k a)}{K_{m}(k a)} .
$$

with $\beta=2 /\left(\Omega^{2}+m^{2}\right)$, and $\rho$, is the density outside the pinch. The quantities $\varphi$ and $x$ are given in terms of Bessel functions.

$$
\begin{gathered}
x=c \frac{J_{m}\left[\left(m^{2} \beta^{2}-1\right)^{1 / 2} \mathrm{ka}\right]}{k a} \\
\varphi=c \frac{m^{2} \beta J_{m}\left[\left(m^{2} \beta^{2}-1\right)^{1 / 2} \mathrm{ka}\right]}{\left(m^{2} \beta^{2}-1\right) k a}+\frac{J_{m}^{\prime}\left[\left(m^{2} \beta^{2}-1\right)^{1 / 2} \mathrm{ka}\right]}{\left(m^{2} \beta^{2}-1\right)^{1 / 2}} .
\end{gathered}
$$

Eq. (5.12) is then solved numerically for the lorgest growth rate. The results for the $m=1$ mode are shown in fig. (5.2). The important differences between the skin current and diffuse current models are that (1) the growth rate does not approoch infinity os $k \rightarrow \infty$, and (2) 
DIFFUSE CURAENT MODEL, M $=$ :

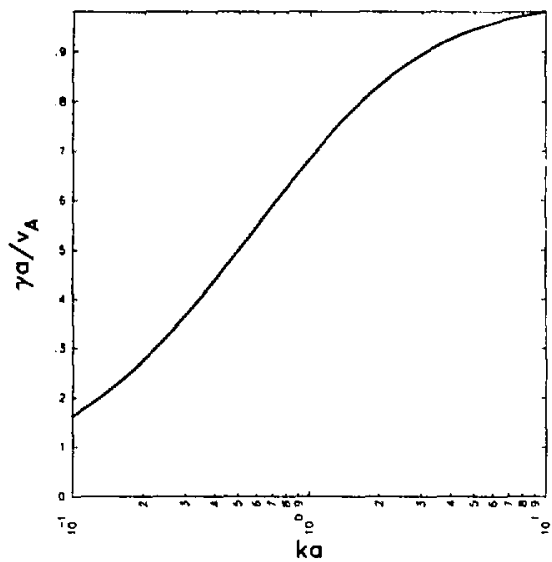

Figure (5.2). Growth rate versus $k a$ for the $m=1$ mode of a diffuse pinch with uniform particle and current densities.

in the previous analysis. If included, it could be expected that they would have the largest effect for long wavelengths, as is seen with the skin current mode.

Closer examination of eqs. (5.12) and (5.13) show that no positive solutions of $\Omega^{2}$ are allowed for $m>1$, making this particular diffuse current equilibrium much more stable than the skin current nodel (see discussion by Taylor. ${ }^{5}$ ) for $m=0$, eq. (5.12) becor 35 indeterminate for on incompressible fluid, and reduces to an equation similar to Bessel's equation for a compressible fluid. With the ratio of specific heat equal to one, the difference amounts to adding an additional term $\left(4 v_{A} \hat{2} / \omega^{2} a^{4}\right) r^{4} \xi_{r}$. Presumably, this equation would need to be solved numerically. Trubnikov ${ }^{7}$ solved for appraximate values of 
ihat with a uniform current density and temperature, but with the particle density varying as $n=n_{0}\left(1-(r / a)^{2}\right)$. He found that for $k a \ll 1, \Omega^{2} \approx 2 \sqrt{3} k a$, and for $k a \gg 1, \Omega^{2} \approx 4 / 3 k a$. His numerical check shaws that the formula for the lower limit is generally applicable up to ka $=1$.

For a pinch with an arbitrary equilibrium profile, eq. (5.4) must be solved numerically for each particular equilibrium. Solutions for growth rates of other interesting compressible equilibria have not been found in the literoture. However. Tayler ${ }^{5}$ does estimate an upper bound for the growth rates for a more realistic density and pressure profile, i.e., one with a uniform temperature. The equilibrium is described by

$$
\begin{aligned}
\rho & =\rho_{0}\left(1-(r / a)^{2}\right) \\
\rho & =\rho_{0}\left(1-(r / a)^{2}\right) \\
\boldsymbol{e} & =\theta_{\theta 0}(r / a) \theta \\
\boldsymbol{d} & =i_{0} \hat{\mathbf{x}}, r \leq a \\
& =0, r>a
\end{aligned}
$$

where outside the pinch the density and pressure ore uniform, and are given by 


$$
\begin{aligned}
& \rho_{1}=\rho_{0}\left(1-(a / b)^{2}\right) \\
& \rho_{1}=\rho_{0}\left(1-(a / b)^{2}\right) .
\end{aligned}
$$

Again, $a$ and $b$ are the pinch and wall radil, respectively. If $\eta$ is Jefined by $(a / b)^{2}=t-\eta$, then the maximum possible growth rate is found to be

$$
\mathrm{n}^{2}=3 / \eta
$$

Thus, it can be seen that as the plasma extends to the wall, and the sackground density becomes small, the maximum growth rate becomes much larger than that of the uniform density pinch. For this reason, the Jrowth rates shown in fig. (5.2) will be considered as the minimum Jrowth rate which can be expected from the MHD theory.

\subsubsection{Finite Larmor radius effect on the growth rate}

If the line density of a pinch is small enough, then finite -armor radius effects can become an important stabilizing mechanism, os is discussed in a number of papers. 15-19 However, none of these or sther papers consider FLR effects in the linear, no $B_{z}, z-p$ inch. The Iffect is caused by an $E \times B$ drift, where the ions experience a slightly different average electric field because of their larger srbifs. than do the electrons. Thus, a charge seporation current is 
built up parallel to the $z$ axis with a perturbed electric field in a radial direction. The $E \times B$ driff for the ions is

$$
=\left(1+a_{\alpha}^{2} / 4 \nabla^{2}\right) \frac{E \times \square}{B^{2}} \text {, }
$$

where $\nabla^{2}$ operates on $\boldsymbol{E}$, and $a_{a}$ is either the electron or ion cyclotron radius. For electrons the second term is negligibly small and is therefore omitted. If the ion orbits are the same order of magnitude as the plasma radius, then the following current results.

$$
\begin{aligned}
\boldsymbol{I}_{\Gamma L R} & =n_{i} e \mathbf{v}_{d i}-n_{\theta} e \mathbf{v}_{d \theta} \\
& =\frac{1}{4} n e a_{i}^{2} \nabla^{2} \frac{\boldsymbol{E} \times \boldsymbol{\theta}}{B^{2}}
\end{aligned}
$$

In order to esilimate a condition for stability, this current is then set equal to the currents which drive the instability, which are the curvature and the $\nabla \boldsymbol{D}$ drifts. This is done by Rosenbluth, et a/ 15 to arrive at a stability condition $\left(k a_{i}\right)^{2} \approx \Omega_{M H O} \Omega_{i}$, where $\Omega_{i}$ is the ion gyrofrequency, with $n_{i}=v_{1 i} / a_{i}$. This result can be further expanded by roting that the MHD growth rate $\Omega_{M H D}$ has a maximum value $v / a$, as shown in the last section. Thus, rewriting this relation in terms of $s$ and $k a$, the following result is obtained, 


$$
\begin{aligned}
(k a)^{2} & =\frac{s}{\pi} \frac{v_{A}}{v_{* i}} \\
& =\frac{s}{\pi} \sqrt{2\left(1+Z_{o v}\right)},
\end{aligned}
$$

where the second line is obtained by agoin using the Bennetf equilibrium condition. Solving for $s$, it is found that the pinch is stable for large ka if

$$
s \leq \frac{\pi(k a)^{2}}{\sqrt{2\left(1+Z_{a v}\right)}} .
$$

With a deuterium pinch which is fully ionized, for example, s must equal 1.6 for $k a=1$, and only 160 for $k a=10$. For longer wavelengths eq. $(5.20)$ is no longer valid since $\Omega_{M H D} \approx k v_{A}(\ln (1 / k a))^{1 / 2}$, which goes to zero for small $k a$. Using this value for $\Omega_{M H O}$, eq. $(5.20)$ becomes

$$
s \leq \frac{\pi}{\sqrt{2\left(1+Z_{o v}\right)}} \frac{k a}{\sqrt{\ln (1 / k a)}}
$$

Eq. (5.21) thus requires that 5 be .1 for $k a=, 1$, and .007 for $k a=.01$. Even these values are probably excessively strict since the wall stabilization effects, which are important for small ka, have not 
leost the short wovelength instabilities can be stabilized for $a_{1} \approx a$. and that longer wavelength instabilities may also be stabilized.

These are heuristic estimates, of course, based on the notion that the perturbed field quantities are small. However, this assumption was violated several times in arriving at eq. (5.20). This equation can only be meant to provide the general scaling feotures of FLR stabilization.

No attempts are made to colculate the diffuse current model MHD growth rates. However, it is possible to place bounds on the growth rates by considering viscous effects. It has been shown by Roberts and Taylor 16 that the FLR effects can be included in the MHD equations by writing them as viscous, off-diagonal terms in the pressure tensor. This adds one further complication, which makes a numerical solution most certainly necessary. It has been shown by Tayler ${ }^{5}$, however, that viscous terms added to the MHD equations decrease the growth rate to zero for small values of the wavelength $\lambda$ (or for large values of $k a$ ). Furthermore, the wavelength of maximum growth rate, $\lambda_{M}$, can be estimated as a function of a collisional mean free path. For a magnetically confined plasma the collisianal mean free path is the Larmor radius. Tayler's expression is

$$
\underline{\lambda}_{g}=\left(\frac{l}{a}\right)^{2 / 3} .
$$


and letting $l=a$, eq. $(5.22)$ becomes

$$
k_{M} \sigma=\frac{\pi}{3} s^{2 / 3}
$$

where $k_{M}=2 \pi / \lambda_{M}$. Growth rates at warenumbers less than $k_{M}$ remrin unchanged, while those at greater $k$ are strongly damped. Nole that upon comparing eq. (5.23) with fig. (5.2) it can be seen that the growth rate goes to zero as $s$ becomes small, which verifies the conclusions based on eq. $(5.20)$.

Use of eq. (5.23) then provides a technique for estimating the maximum grawth rate expected with izo elfects. One first determines the value $s$ by eq. $(1.6)$, determines the value of ka which gives the maximum growth rate by using eq. (5.23), and then matches $k_{M} a$ with either fig. 5.1 or fig. 5.2 to obtain the growth rate.

Several other papers should be mentioned before continuing. The first is by stringer ${ }^{18}$, whe calculates analytic solutions for the growth rates near the $\boldsymbol{k} \cdot \boldsymbol{E}=0$ surfaces of a cylindrical screw-pinch. He also provides a technique for determining the growth rate with FLR effects, given the MHD growth rate, provided, of csurse, that $a_{i} \ll a$. Because of the included $B_{z}$, his results do not apply to this work. Trubnikov and Zhdanov ${ }^{19}$ consider an anisotropic pressure tensor which includes FLR effects in determining the growth rate of an $m=0$ linear pinch (no $B_{-}$) with the approximation that $k a \gg 1$. Their result is 


$$
\omega=-\frac{1}{2}\left(k a_{i}\right)^{2} \omega_{c i}+i \sqrt{\left|k g_{0}\right| 1-\left(k a_{i}\right)^{4} \omega_{c i}{ }^{2} / 4}, k a \gg 1,
$$

where $\omega_{e} i$ is the ion Lormor frequency, and $g_{e f f}=(2 p / r p) \approx v_{1} i^{2} / r$. This equation is of limited volue in the present experiment since ka is observed to be $\approx 1$.

From these considerations several general conclusions can be drawn. (1) As shawi by eq. (5.23), short wavelength growths are most strongly damped by FLR. effects. (2) Those modes with the finer structure, i.e., the higher order $m$ modes will be more strongly stabilized. (3) If FLR stabilization can occur, it must be for $a \sim a_{i}$.

5.1.5. Comparisons of observed with calculated linear grawth rates

As mentioned in the last chapter, growth rates were difficult to measure for the $m=0$ mode. It was only possible to place lower bounds on the growth rates. With the Trubnikov model, ${ }^{7}$ for ka $\approx 1$, which was the most commonly observed wavelength, $\gamma a / v_{A}=1.9$. Experimentally, two separate $m=0$ growth rates were observed for each shot; one several arders of magnitude less than, and later one much greater than the predicted value. For the shots with low $s$, the pinch stayed quiescent, or slowly growing. for up to $1 \mu \mathrm{sec}$, before abruptly breaking in a sausage-like instability. This sausage-like instability was observed to grow at a rate much faster than that predicted by the 
For the $m=1$ mode, a comparison can be made between the data in table 4.2 and fig. (5.2). Becousc of the relalively few shots that exhibiled the kink instability, the point scotter is largo. However, several general features can be deduced from such o plot as is seen in fig. (5.3); (1) The experimenlally observed rotes are roughly a factor of ten less than those predicted by the theory, and (2) No kinks are observed for ka>3. This last statement is possibly not entirely correct. It is possible for the kink mode to non-linearly couple to the sausage mode so that o short wovelength internal kink might evolve into an external sausage instability. ${ }^{20}$ It was not possible to resolve this effect from the froming camera imoges becouse of the finer structure at the shorter wavelengths.

5.2. The pinching dynamics and non-MHD effects

Recently, there has been much discussion in the literature of additional effects neglected by Anderson,et a/21 and Leontovich and Osovets 22 on the dynomics of the pinch compression. These odditions enable the theory to better explain the final states of the pinch, as well as to predict the abrupt disruptions observed in almost all pinches. Since these ideas hove direct bearing on the observed dynamics and instabilities, they will be Jiscussed in this section in the context of this experiment. 


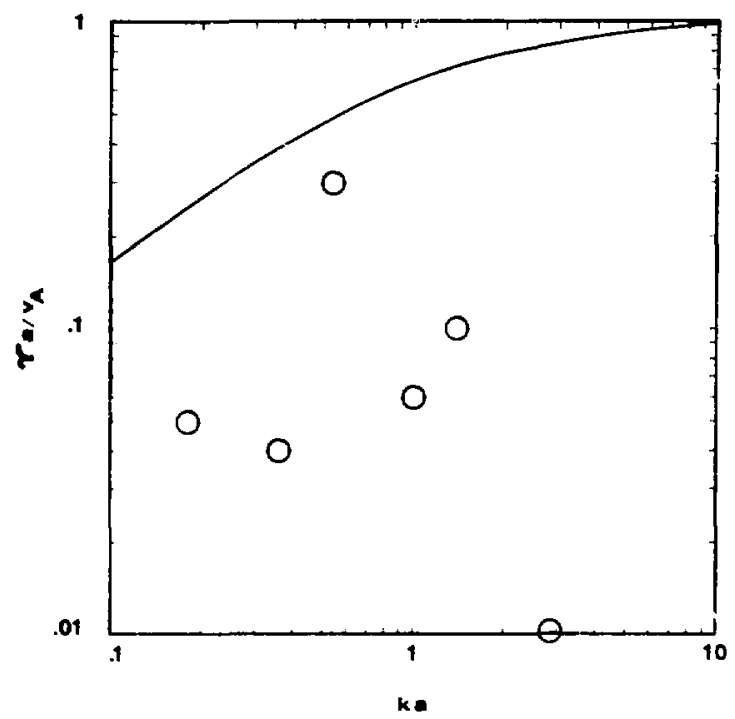

Figure 5.3. Normalized $m=1$ growth rate versus ko. The solid line is the resull of the Tayler theory, and the points come from lable 4.2.

\subsubsection{Pinching dynamics}

The snow-plow model deseribed in chopter 1 is derived by considering the forces on o current carrying sheet that is accelerated inwards by its self-generated magnefic field, accruing mass as it sweeps in. The particle pressure of the gas is neglected, and therefore it is not possible to predict the final radius, or subsequent behovior of the pinch. The derivation of the Leonotovich and Osovets equation 22 goes one step further and includes the porticle pressure. Thus, the model is able to predict o final radius and tamperature, o 
subsequent expansion, and a recompression. The snow-plow model is limited, however, in that it considers the plasmo to reside in a thin, nollow shell. It is therefore not possible to correctly model the mixing and heoting that takes place at peak compression. This problem is corrected by considering a shock-model, as done by Allen. 23 Potter ${ }^{24}$ improved on the shock model with a slug model, which includes the effects of a cylindrical strong shock preceding a piston of plasma (the slug) which collapses to the axis.

However, even these improved techniques do not include many effects which become important in the loter stages of the compression. Axial streaming of plasma during the compression can lead to removal of aggregated material, thereby allowing much higher compression than in a symmetric Z-pinch.24.25 This effect, which is important in the plasma focus, also pertains to this experiment becouse of the conical distribution of gas prior to pinching. As the pinch current is increased, there is on axial component of the compression directed towards the anode, as is discussed in chapter 3 . This effect can also lead to enhanced heating, as is noted by Vikhrev and Korzhavin, ${ }^{25}$ where localized. low-density regions with higher electron drift velacities are established. If the density becomes low enough, there is an anomalously high resistivity, and rapid heating and expansion.

Ary mechanism which reduces the line density locally could be a cause of increased resistivity. Thus, for example, it is also possible 
region, could also cause an onset of anomalous resistivity, and increased heating. For the incompressible caso, $\nabla_{*}=0$ gives an axial flow 0 :

$$
v_{2}=\frac{i}{k r} \frac{\partial}{\partial r}\left(r v_{r}\right)
$$

For $m \neq 0$, there is also on azimuthal term - $m v_{\theta} / k r$, which decreases the axial flow. Thus, this effect could be expected to be strongest for $m=0$ modes, and perhaps this is a reason that this mode is the most commonly observed discuptive mode, despite the fact that the ideal MHD theory shows that it can be stabilized by a sufficiently diffuse profile.

There are a number of micro-instability interactions which will lecd to enhanced resistivity of the pinch, and therefore must be included in a dynamical theory of the later compression stages. Liewer and Kral127 discuss three types: the Buneman, the ion acoustic, and the lower hybrid dift instabilities. Vikhrev and Korzhavin 25 list these and four additional types, conveniently compared in a table, which can affect the pinch dynamics. Each becomes important when the electron drift velocity exceeds a specific threshold for that instability. The lower-hybrid drift instability becomes important when $v_{d .}>v_{f i}$, where $v_{f i}$ is the ion thermal velocity. The ion-acoustic instability, which also has a low threshold, turns on when the drift 
restriction probably eliminotes this instability for consideration in the earlier stages of the pinch dynamics since $T_{i} \gg T_{\text {a }}$ during the snow-plow portion of the compression. The Buneman instability is also probably of lesser importance since it requires that $v_{\text {do }}>v_{t e}$. The only other micro-instability that has a low turn-on threshold is the electron-acoustic instabili'y, which has an identical threshold, and gives an identical resistivity as that of the lower hybrid drift instability. Therefore, it is sufficient to consider only the fower hybrid drift instability for $v_{d e} \approx v_{t i}$.

As was shown in the derivation of eq. (1.8), the criteria that the drift velocity exceed the ion thermal velocity for the lower hybrid drift instability con be written in terms of the FLR parameter $s$, which in turn is written in terms of a line density $N=\pi n a^{2}$ by the use of the Bennett relation. Similarly, the thresholds for the other instabilities can also be written in terms of line densities. These are olso tabulated by Vikhrev and Korzhavin 25 for deuterium. For higher $Z$ gases the line density thresholds are complicated somewhat by their dependence on the overoge ion charge, which is temperature dependent. Thus, for argon, it was shown that the onset of the lower-hybrid-drift instability is relaxed somewhat from that of deuterium. The magnitude of the anomalous resistivity caused by the micro-instabilities san be appreciably larger that: that of the classical coulomb resistivity. The anomolous resistivity $\eta$ is written in terms of the classical resistivity $\eta_{c}$ as 


$$
\eta=\eta_{C}\left(1+\nu_{0 f f} T_{0 i}\right)
$$

where $\nu_{\text {off }}$ is the effective collision frequency, and $T_{0} i$ is the classical electron-ion collision time. The values of veff are given by Vikhrev and Korzhovin, 25 where for the lower hybrid drift instability it equals $\left(v_{d o} / v_{t i}\right)^{2} \omega_{L H}$, with the lower hybrid frequency $\omega_{L H}=\sqrt{\omega_{c \theta} \omega_{c i}}$. The product $\nu_{\theta f} T_{\theta i}$ is typically of order $10^{4}$ to $10^{B}$. depending on the temperature and line density.

In order to demonstrate what effect anomalous resistivity has on the expansion of the pinch, consider an equilibrium pinch of radius a that suddenly becomes anomalously resistive. The external PdV work goes into resistive heating of the pinch and radiative losses. Thus,

$$
\begin{aligned}
\text { PaV } & =P_{\Omega}-P_{R} \\
\frac{\mu_{o} I^{2}}{8 \pi^{2} a^{2}} \frac{d}{d t}\left(\pi a^{2}\right) & =\frac{I^{2} \eta}{\pi a^{2}} .
\end{aligned}
$$

The radiative term is dropped because it is much smaller than the anomalous heating term. The pressure balance relation was also used to express the particle pressure of the first line of the equation in terms of a magnetic pressure. Simplifying, the following is obtained. 


$$
\frac{d a}{d t}=\frac{4 \eta}{\mu_{0} a}
$$

For a pinch of radius $0.5 \mathrm{~cm}$, and with the resistivity $\eta=10^{4} \eta_{C}$, which is low in many instances, the expansion rate is

$$
\begin{aligned}
\frac{d a}{d t} & =37 \mathrm{~mm} / \mathrm{ns}, \quad T=20 \mathrm{eV} \\
& =0.1 \mathrm{~mm} / \mathrm{ns}, \quad T=200 \mathrm{eV} .
\end{aligned}
$$

Thus, it can be seen that the expansion of the pinch by anomalous heating is indeed very fast.

One other physical effect has been found to be very importari in more correctly describing the dynamics of a pinch, is consideration of the radiation losses, as alluded to in eq. (5.27). Sheorer ${ }^{28}$ has shown that if the radiation losses of the plasma exceed the ohmic heating. then the pinch cools and collapses to a high density filament. This effect is olso discussed by Vikhrev ${ }^{26}$ who indicales that the pinch can collapse only until the density becomes high enough that it radiates as a black-body radiator. The condition that the radiative cooling rate exceeds the ohmic heating rate is expressed in terms of the Peose-Braginski i current $I_{P E}, 29,30$ namely thot 


$$
\frac{1}{a} \frac{d a}{d t}=\frac{3}{4} \frac{1}{\tau_{R}}\left[\left(\frac{I_{P B}}{I}\right)^{2}-1\right] \text {. }
$$

where the radiation time constant $\tau_{R}$ is defined as

$$
\tau_{R}=\frac{3 N k T}{P_{R}}=\frac{3}{4} \frac{I^{2}}{P_{R}} .
$$

Eq. (5.30) was derived from eq. (5.27) using the Bennett equilibrium.

With Coulomb resistivity, the Pease-Braginski current is of order $1.5 \mathrm{MA}$ for decterium, and $110 \mathrm{kA}$ for argon, being also dependent on the density profile. If, however, the resistivity is not classical, but rather anomalous, the Pease-Braginskii current becomes very high, and the pinch expands, which is consistent with eq. (5.29).

Thus, a complete description of the dynamics of the $2-p$ inch must include these three effects; axial flow, anomalous resistivity, and radiation, if it is to successfully explain the complex phenomena often experimentally observed. Recent 1D numerical calculations with LASNEX ${ }^{31}$ have been done by Nielsen, 32 and show that an enhanced resistivity prevents the pinch from attaining the small radii found whin just radiation and Coulomb resistivity are considered. Vikhrev and Korzhavin 25 modeled the plasma focus and found the rapid hisating and expansion, and subsequent recompression with the anomalous resistivity playing a large role in ihe dynamics. They have explained 
many of the effects observed with the plasma focus, but they also do not consider radiation effects.

5.2.2. The volfage spike and the sausage-like disruption

Voltage spikes, dips in the pinch current, $x$-ray bursts, and, for deuterium, neutron emission, have long been observed in pinches and pinch-like devices. It has long been known that the neutrons generated are not thermonuclear in origin, 21 but have directed energies and are therefore not useful for controlled fusioll. There have been many proposed explanations, which can fit into three broad categories; (1) Inductive effects due to the norrowing of the pinch column, Anomalously resistive effects, and (3) Electromagnetic effects. The intent of this section is to discuss each of these in the context of the present experiment.

The induced voltage across the pinch electrodes has three possible sources:

$$
\begin{aligned}
\mathscr{E} & =\frac{d}{d t}(L i)+i R \\
& =L \frac{d i}{d t}+i \frac{d L}{d t}+i R .
\end{aligned}
$$

The inductance $L$ per unit length of the pinch is $L=\mu_{0} / 2 \pi \ln (b / a)$, where $a$ and $b$ are the pinch and wall radi $i$, respectively. Neglecting 
the resistive effects for the moment, and noting that $a=a(t)$ and that di/dt is small, the induced voltage is

$$
\mathscr{E}=i \frac{d L}{d t}=i \frac{\mu_{0} l}{2 \pi} \frac{b}{d} \frac{d y}{d t} .
$$

The velocity dy/dt is the pinch radius collapse velocity, normalized to the initial wall radius, and $l$ is the pinch length. From fig. (3.5) this velocity is found to be of order $1.0 \times 10^{6} \mathrm{~cm} / \mathrm{sec}$. Thus, to produce a voltage spike of $16 \mathrm{kV}$, which was often seen, with a current of $100 \mathrm{kA}$, and a rodius of $0.3 \mathrm{~cm}$. requires that the filament radius be $0.1 \mathrm{~mm}$. Filaments of this size, which would be produced by a radiative collapse, were not abserved with the framing camera, nor with the interferometer. Furthermore, the calculations of chap. 2 indicate that the plasma filament should still be visible, if it is present. It is therefore very doubtful that filamentation was the cause of the voltage spikes in this experiment. Those filaments which do sometimes remain, such as the one seen in fig. 4.2, appear to be less dense, perhaps being the remainder after the outer layers have been thrown of $f$.

Bernard, et $01 ., 33$ were the first to suggest that anomalous resistivity might be the cause of the voltage spikes, and disruptions in the plasma focus, and other z-pinch like devices. As discussed in the last section, axial flows induced by non-axially uniform confiaurations (i.a.. the olasma facus or this axnarimont) nr thase 
axial flows arising from on $m=0$ instability, can cause regions of lawer line density, where the locol eloctron drifl velocity exceeds the ion thermal velocity, making the plasma unstable to a lower hybrid drift instability. The resulf is that the plasma quickly heats and exponds to a lower density, and emits less visible light, since the bremsstrahlung is proportional to the square of the density.

Consider now eq. (5.32) with the resistive term dominating. Using the same parameters os used with tha inductive case, $R$ need only be . $16 \Omega$ to produce the $16 \mathrm{kV}$ voltage spike. Thus, $\eta / \eta_{C}=30$ for $T=20 \mathrm{eV}$, and $10^{3}$ for $T=200 \mathrm{eV}$, considering only o portion of the pinch length to be onomolously resistive. Both of these values are approximately a foctor of ten less thon the resistivity estimated for the lower hybrid drift instability, but they do indicate that anomalous effects could very well be the cause of the voltage spike.

One additional observation helps substantiale the concept that axial flows lead to reduced line density and localized micro-instabilities. Namely, the break-up of the pinch was observed to often form near the gas valve opening in the cathode, where the gas distribution transitions from a cylindrically shoped to a conically shaped jet. Nearest the cathode the pinching is primarily radial. Further away there is an axial component directed away from the cathode. Thus, in the transition regian between the two there will then be a region of reduced density, i.e., one where more gas is flawing out than coming in. Therefore, any rapid heating could be 
expected to start in this region. This phenomenon was seen with most of the pinches which broke up in a sausage-lile instability. See, for example, figs. $3.8,3.10,4,2,4.3$, and 4.5 .

The third possible explanation for the voltage spi 2 , and break-up of the pinch is that it is associated with the displacement current, wich is not included in the ideal MHD equations. The addition of the displacement current then allows electromagnetic effects. Trubnikov and Zhdanov ${ }^{34}$ write the cylindrical wave equation, and find a solution for a sudden break in an infinitesimally thin wire with curreit $J_{0}$ as

$$
E_{z}(r, t)=\frac{2 J_{0}}{c\left(c^{2} t^{2}-r^{2}\right)^{1 / 2}} .
$$

This solut on has the properties of an initially very strong axial electric field, going to infinity at $r=c t$. Thus very strong electric fields are present which accelerate electrons and ions across the magnefic field (assuming $E \gg B$ ). X-ray bursts and neutron yields are then associated with the interaction of tiese accelerated particles with the plasma on either side of the discontinuity.

Picke Is $s^{35}$ suggests that an electro-magrietic effect causes B-field defachment from the current sheet and penetrotion of the plasina, resulting in strong displacoment fields, as in eq. (5.34). This hypotheses is based on clues from the LLNL, and the Gribkov, et a ${ }^{36}$ 
before peak compression. This mechanism could possibly also explain why the voltage spike does not always coincide with the peak compression. This theory has yet to be worked out in detall, however.

It wos not possible to verify either of these two theories with the present experiment, and therefore no conclusions are made concerning their validity. It is obvious, however, ihat both approaches can include anomalous resisitivity effects, and are possibly even required to explain the sudden current discuption used to produce the wave of eq. $(5.34)$.

References for Chapter 5

'I. B. Bernstein, E. A. Frieman, M.D.Kruskal, and R. M. Kulsrud, Proc. Roy. Soc. (London) A223, 348-360 (1954).

${ }^{2}$ G. Schmidt, Physics of High Temperature Plasmas (Academic, New York, 1979!, 2nd. ed., p. 120.

3. P. Goedbloed, and H. J. L. Hagebeuk, Phys. Fluids, 5, 1090 (1972).

${ }^{4} M$. Kruskal and M. Schwarzschild, Proc. Roy. Soc. (London) A223, 348-360 (1954): also in Magnetohydramic stability and Thermonuclear Containment, edited by A. Jeffrey and T. Taniuti (Acodemic, New York, 1966), pp. 63-75. 
5R. J. Tayler, Proc. Phys. Soc. (London) B70, 31 (1957).

6R. J. Tayler, Proc. Phys. Soc. (London) B70, 1049-1063 (1957): olso in Jeffrey and Taniuti, Ibid., pp. 149-163.

${ }^{7}$ B. A. Trubnikov, in Plasma Physics and the Problem of Controlled Thermonuclear Reactions, Vol. I, edited by M. A. Leontovich (Pergamon, New York, 1961), pp. 349-361.

8V. D. Shafranov, in Plasmu Physics and the Problem of Controlled Thermonuclear Reactions, Vol. II, edited by M. A. Leontovich (Pergamon, New York, 1959), pp. 197-215.

${ }^{9} \mathrm{~V}$. C. Shafranov, in Plasma Physics and the Froblem of Controlled Thermonuclear Reactions, vol. IV, edited by M. A. Leontovich (Pergamon, New York, 1959). PP. 71-80.

${ }^{10} \mathrm{~B}$. B. Kadomtsev, in Reviews of flasma Physies, Vol. II, edited by M. A. Leontovich (Consultants. New York, 1966), pp. 153-199.

1'K. Hain and R. Lust, Z. Naturforsch. 13a, 936 (1958).

12 W. A. Newcomb, Ann. Phys. (N. Y.) 10,232-267 (1960): olso in Jeffrey and Taniuti, Ibid., pp. 169-204.

$13 \mathrm{~J}$. P. Friedberg, Phys. Fluids 13, 1812 (1970).

14Kadomtsev, Ibid., p. 177.

i5M. N. Rosembluth. N. A. Krall, and N. Rostoker, Nuc. Fusion Suppl. A1 (1962) 143; also in Jeffrey and Taniuti, Ibid., p. 131. 
16K. V. Roberts and J. B. Taylor. Phys. Rev. Lett. 8, (1962) 197.

${ }^{18} \mathrm{~T}$. E. Sitringer, Nucl. Fusion 15, (1975) 125.

${ }^{19}$ B. A. Trubnikov and S. K. Zhdonov, Sov. Phys. JETP 37, (1973) 625.

$20^{\circ}$. B. Kodom!sev, Ibid., p. 168 .

210. A. Leontovich and S. M. Osovets, J. Nucl. Energy 4, 209 (1957): also discussed, with improvements, by L. A. Artsimovich, Controlled Thermonuclear Reactions, edited by A.C. Kolb and R. S. Peose (Gordon and Breoch. New York, 1964), pp. 123-128.

23 J. E. Allen, Proc. Phys. Soc. 70 (1957).

${ }^{24}$ D. Potter, Nucl. Fusion, 18,813 (1978).

${ }^{25} V$. V. Vikhrev, and V. M. Korzhavin, Sov. J. Plasma phys. 4, 411 (1978).

26V. V. Jikhrev. Sov. Phys. - JETP Left..27, 98 (1978).

27P. C. Liewer and N. A. Krall, Phys. Fluids 16, 1953 (1973).

$28 \mathrm{~J}$, W. Shecrer, Phys, Fluids 19, 1426, (1976).

${ }^{29}$ R. S. Peose. Proc. Phys. Soc. Lond. A 10, 11 (1957). 
${ }^{30} \mathrm{~S}$. I. Braginskii, Soc. Phys, JEJP 6, 494 (1958).

${ }^{31}$ LASNEX is a 2D lagrangian code developed at LLNL for study of laser pellet implosions. It hos a full radiation package, and has been modified to study enhanced resistivity effects.

$32 \mathrm{P}$. Nielsen, private communication: Ph.D. thesis, to be published as UCRL, foll 1980 .

${ }^{33}$ A. Bernard, A. Coudeville, A. Jolas, J. Launspach, and J. de Moscureou Phys. Fluids 18, 180 (1975).

${ }^{34}$ B. A. Trubnikov and S. K. Zhdonov, Sov. Phys.- JETP 43, 48, $(1978)$.

35w. Pickels, private communicotion: also article to be published in J. App 1. Phys.

${ }^{36}$. A. Gribkor, O. N. Krokhin, G. V. Sklizkor, N. V. Filippor, and T. I. Filippova, Sov. Phys. - JETP Letl. I8, 5 (1973). 


\title{
CHAPTER 6
}

\author{
Summary
}

6.1. Results

The gool of the experiment was to observe FLR effects in the linear z-pinch. The experiment was successful to a large extent in that the scaling of the instability types was clearly observed to be dependent on the electron line density of the pinch, o parameter which is directly related to the strengtin of the FLR effects. No theory was construcled which includes these effects becouse of the difficulties involved in using o fluid theory when the ion orbits ore large. 
In the first chapter the Finite Larmor radius scaling was developed. It was found, by using the pressure balance condition, that the number of pinch orbits in the radius is proportional to the square root of the line density and independent of the temperoture if the plasmo is completely ionized. For higher $z$ plasmas, which are usually not completely ionized, there is a mild depedence an the average effective chorge of the ion. This $Z_{\text {eff }}$ dependence implies that low temperature, high $Z$ plasmas can have strong FLR effects of much higher line densities than hydragen.

It was also found in the first chapter, that to avoid regimes where micrainstabilities exist it is important to keep the electron line density high enough that the electron drift velocity not exceed the ion thermal velocity. This restriction places severe limitations on the amount of FLR stabilization thot could be ochieved, but suggests that there might exist o stability window between the conflicting requirements of these two mechanisms. For higher $Z$ materials it was found that the line density threshold is relaxed somewhat.

The experiment, which differed significantly fromeorliar pinch devices, is described in the second chapter. The apparatus consisted of an evacuated pinch chamber with gas puffed on-axis through a hole in the cathode. The pinch bank was fired before the gas hod time to reoch the wolls. Therefore the dynamics of the pinch were somewhot different from the previous snowplow pinches where the pinch current starts at the wall. With this techniaue it was dossible to maintain the line 
density low enough that FLR effects are important, and yet have a local density high enough that the gas could breakdown.

Spatial electron dansity measurements were made of a number of the shots with a CW laser, time resolved with a gated intensifier and TV camera. A smoll code wos developed to Abel invert the interferometer data, and to do least-squares fits to various density profiles of the computed radial densities. However, since density measurements were not available for all shots, it was necessary to provide a means of scaling the shots by density from the control settings of the experiment. This was done by calculating the pulsed gas valve output as a function of time, so that a knowledge of the timing and plenum fill pressures allowed determining the pinch density.

The other primary diagnostic of the experiment wos the STL framing comero. To understand haw the plasma brightness in the visible spectrum varied with density and temperature, the relative brightness of the line radiation, the recombinotion, and bremsstrahlung radiation was calculated. This calculation was complicated by the need to use and argon plosmo to provide sufficient light for the froming comera, and therefore required calculation of the average ion charge. The average ion chorge, which is a function of temperature, was determined with an approximate technique (good to $10 \%$ ) that is primorily saha-like af lower ionization levels, and corona-like at the higher levels. From these calculations the brightness of the plasmo was found to stay relatively constant over a large range of temperatures. At low 
temperatures line radiation was found to dominate, and as higher ionization levels are attained, the bremsstrahlung dominates. The recombination radiation was not found to be significant. Furthermore, the intensity of the line radiation is found to scale as $n$, and the bremsstrahlung as $n_{i} n_{0}$ for a given temperature.

In the third chapter the density voriation of the gas jet between the electrodes is estimated from supersonic jet studies to be $(x / d)^{-6 / 5}$, along the axis, and to vary radially out to $45^{\circ}$ as $\cos ^{2} \theta$, where $\theta$ is measured about the orifice. The breakdown of the gas was observed to occur at the outer edges of the gas, thus causing the initial plasma to have cone-shaped distribution, with the apex of the cone at the cathode. As the pinch current rises, the plasma is observed to pinch to a straight column, reaching peak compression at the cathode end. This non-uniform radial compression then has an axial component directed towards the anode, the magnitude of which can be as large as 0.4 times that of the radial velocity, depending on the angle of the compressing plasma near the axis.

The time to pinch, and the velocity of the imploding shock wave are calculated with the well-known snowplow model, both with linearly increasing and constants currents. For the canstant current case the time to pinch is found to be proportional to $\rho_{0}^{-1 / 2}$, rather than the more usual $\rho_{0}^{-1 / 4}$ scaling found for the linearly increasing current. A numerical solution was also done with a current which rose linearly, and then loveled off to a constant current before pinching. For this 
case, a scaling law was found that relates the time to pinch to that of the constant current case. Furthermore, the scaling of the time to pinch for this equation, i.e., $t_{p} \propto \rho^{-1 / 2}$. was also experimentally observed.

The formotion and life of the gas-puff, and the gun initiated pinches ore also shown from framing camera images. Several gases were used, although the most success in obtainirg straight, slowly growing pinches was with the higher $Z$ gases. No straight, long lasting hydrogen pinches were observed. Abel inversions of the interferograms showed that the plasma density resides primarily in a shock region during the compression phase, but becomes a Bennett profile at peak compression. That is,

$$
n(r)=\frac{n_{0}}{\left(1+(r / a)^{2}\right)^{2}}
$$

where $n_{0}$ and o are constants.

With almost every shot, near peak compression. a large voltage spike ( 2 to 3 times the charge voltage) appeared across the pinch electrodes. The pinch column was observed to go dork at this time in a small region located usually near the cathode. Coinciding with the voltoge spike a hard $X$-ray spike was also always observed. The X-ray energies were measured by absorption techniques to be of order $\theta$ limes $1 /$ 
The pinch usually appeared to break in sausage-like $(m=0)$ disruption, with a growth rate at least an order of mognitude higher than those predicted by the MHD theory. Often, however, the pinches wou : d remain straight for up to 1 usec before suddenly disrupting. Thus, it is concluded that the instablitity is not ideal MHD, but must include effects neglected in the MHD theory.

The $m=1$ external kink mode was also observed, al though it was not seen nearly as often as the sausage-like. The observed growth rates were found to have growth rates approximately a factor of ten less than those predicted by the diffuse WHD theory with a quadratic pressure profile.

The type of instability observed was found to vary with the electron line density, which is proportional to $s$, the number of ion orbits in the pinch diameter. For low densities, pinches were observed which expanded of one end of the pinch column before reaching maximum compression of the other. At higher densities, the more usual sausage-like intrubilities were observed. At still higher dansities pinches were observed that remained straight for up to $1 \mu s e c$, before slowly expanding. At the highest densities studied, the more typical kink and sausage instabilities were observed. It is concluded that at the lowest line densities the microinstablitities dominate the development of the pinch, whereas at the higher line densities, MHD instabilities dominate. For the high $z$ plasmas a stability window 
possibly exists between the two regimes, which is non-existent for the low $Z$ plasmas.

Growth rates for the $m=0$, and the $m=1$ modes were calculated and plotted for the skin current model, and for a diffuse current, quadratic pressure proflle model. The skin current model, which contains no $B_{z}$, predicts growth rates which differ significantly from those usually discussed with the screw pinch, in that as $k a \rightarrow 0$, the growth rate $\rightarrow 0$, whereas for the screw pinch it remoins finite. The effect of adding a diffuse current is to limit the growth rate at large ka to $\gamma a / v_{A}=1$, whereas with the skin current model $\gamma a / v_{A} \rightarrow \infty$.

When considering FLR effects by adding viscous terms to the MHD equations, it is found that th short wavelength growth rates are strongly reduced, and that the wavelength of the maximum growth rate, written in terms of the wavenumber, is $k_{M} a=\pi / 3 s^{2 / 3}$. For wavenumbers less than this value, the growth rates remain unchanged.

Finally, it was concluded that anomalous resistivity effects probably play the largest role in causing the large voltage spikes, and associated $x$-ray bursts that are observed in this experiment. Purely inductive effects caused by simple radiative cooling and compression of the plasma, are simply not observed. Rather, the pinch is found to expand to a lower density, larger diameter pinch, characteristic of a pinch which is quickly heated by anomalously resistive heating. 


\subsection{Further work}

Probably because of the known instabilitiy of the linear z-pinch, there have been very few calculations done on linear growth rates of the diffuse pinch with anything more than a quadratic profile, for which a transcendental equation for the dispersion relation can be obtained analytically. An important extension to this work would be a numerical solution to the linearized MHD equations for the growth rates using a Bennett profile, allowing viscous effects, and preferably not requiring incompressibility. Furthermore, since most pinches do not extend to the wall, solutions to the free-boundary pinch should be found, in spite of the difficulties with boundary conditions at the plasma-vacuum interface. FLR effects could be added by considering them as viscous effects. Or, they could be included in the MUD equations by starting with the Vlosor equation and not neglecting the FLR terms, as is commonly done in obtaining the ideal MHD equations. A similar approach is done by Kerbel' to calculate the magnitude of the FLR effects for the diffuse-current screw pinch with the ordering that $B_{z} \gg B_{\theta}$

With these proposed calculations, it would be hoped a more precise dividing line between FLR stabilization, and microinstabilities could be established. 
$-153-$

References for chapter 6

'G. Kerbed, private communication; PhD thesis under supervision of W. A. Newcomb, to be published as a UCRL, Sept. 1980. 


\section{APPENDIX A}

\section{Pulsed Gas Valve Construction and Gas Flow Dynamics}

This appendix contains a discussion of the Cheng style gas valve construction and operation, and of the gas dynamic calculations used in preparing fig. 2.5 in chapter 2 .

A.1. Construction and operation of the gas valve

The construction of the gas valve is shown in fig. 2.4 of chapter 2. The body and base of the gas valve are constructed of Lexon to electrically insuloted the plosma gun, and to provide mechanical strength. Several valve bodies which were made of plexiglass cracked during use. 
The primory coil had $51 / 2$ turns and was coristructed from a $95 \mathrm{~mm}$ wide, $0.5 \mathrm{~mm}$ thick copper strip. The leads are B \& $S$ no. 8 gage copper wires with the ends pounded flat and silver-soldered of right angles to
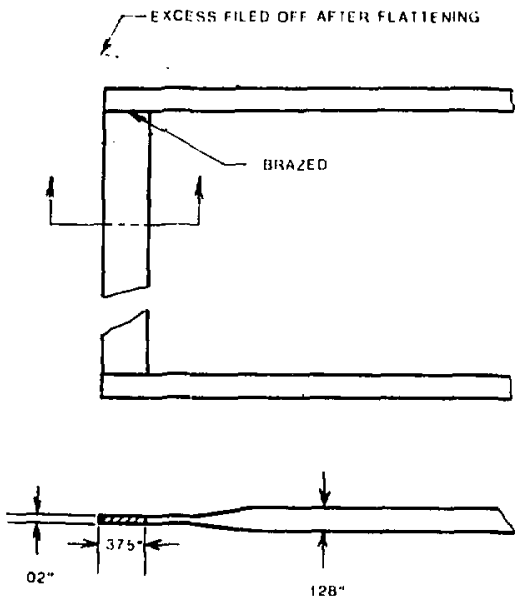

Figure A.1. Construction of the primary coil.

the copper strip. The leods and connecting strip were covered with averlapping mylar tape to prevent electrical breakdown. The coil was wound on a form, was covered, and filled with epoxy. In the process of winding both leads were bent back $180^{\circ}$ and spaced $180^{\circ}$ apart on the circumference. Atter the epoxy hardened, the coil and volve base were epoxied into the gas valve body. The leads coming out the top were 
electrically insulated from the cover plate by $1 / 4$ " D polyflo vinyl fubing.

The secondary coil can be either a coil-wound bobbin, or a copper disk, as shown in fig. 2.4 of chapter 2 . The copper disk was found to be satisfactory becalse its driving force was more than sufficient, and because there was some concern about jitter caused by the movement of the windings with a coil-wound babbin. Since the magnetic field diffusion time through copper is relatively slow the need to slot the valve stop to reduce its eddy currents was eliminated.

The primary coil current was supplied by two $7.5 \mu \mathrm{F} / 20 \mathrm{kV}$ copacitors normally charged from 10 to $16 \mathrm{kV}$. Fire and crowbar ignitrons were used so that the current rose to a maximum in $10 \mu s e c$ and decayed in $100 \mu \mathrm{sec}$. The peak current ranged from 10 to $16 \mathrm{kA}$. The capocitors were located about $8 \mathrm{~m}$ from the gas valve. For this reason the current profile was determined primarily by the external inductance of the connecting transmission line.

The ittter in the valve opening time was measured with a gas breakdown probe lacated $3 \mathrm{~cm}$ downstream from the valve opening and found to be $\pm 5 \mu \mathrm{sec}$. Some of this ijtter might be attributed to turbulence and breakdown characteristics of the gas. Therefore one cruld expect smaller iitter times than measured from the breakdown probe. 
The delay in the gos valve opening and open time was measured by shining a He-Ne laser an the disk/O-ring seal. and by using a photomultiplier tube to measure the transmitted light os the valve opens. Figure A.2 shows on oscilloscope trace of the light output and the primory current as a function of time with a 12 kV capacitor

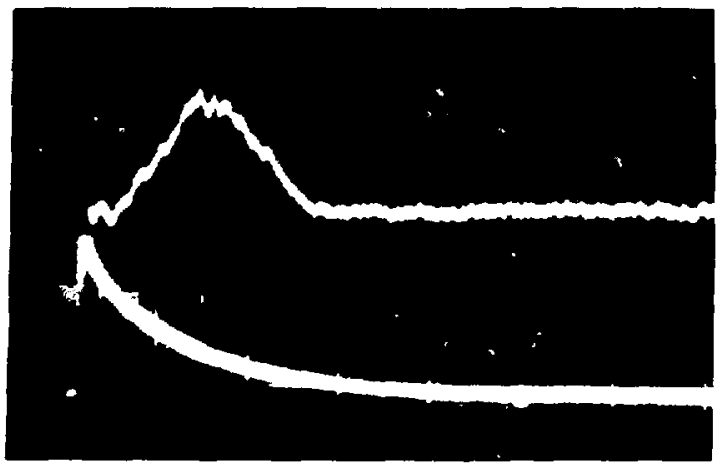

Figure A.2. Oscillope troces of the gas valve stop position and primary coil current. The upper trace is the photomultiplier output of a laser beam directed across the vaive at the O-ring seal. The lower trace is the current at $8 \mathrm{kA} / \mathrm{div}$. Both horizontol scoles one $100 \mu \mathrm{sec} / \mathrm{div}$.

charge. The opening delay was found to be $40 \mu \mathrm{sec}$, and was only slightly less at higher voltages. The light output indicates that the valve disk is bounced of the stop, and refurns to the seal rether 
quickly. The length of time the valve was open was found to be indepandent of the plenum pressure below about eight atmospheres. At higher pressures the gas provided an additional restoring force which more quickly closed the valve. The lime was found to be very dependent on the stop position, however. At a $12 \mathrm{kV}$ capacitor chalge the disk velocity was measured of $1.1 \times 10^{3} \mathrm{~cm} / \mathrm{sec}$. The omount of time the volve remoins open is the time for the disk to reoch the stop and bounce bock.

The total gas valve output was measured with orgon at various plenum pressures and stop settings, for on early version with o somewhat different primory coil construction. The output ronged from .2 to $20 \mathrm{Atm}-\mathrm{cm}^{3}\left(5 \times 10^{18}\right.$ to $5 \times 10^{20}$ porticles. $)$ The results of these measurements are given in fig. A.3. The decrease in output at higher pressures is apparently due to o higher restoring force on the disk which more quickly closes the valve.

\section{A.2. Gos Dynomics}

The gas output os a function of time can be estimated from rate equations. Consider the geometry of fig. 2.4, which shows the gas valve and gun assembly. As the valve opens, the gas enters on intermediate chamber. building up pressure, and escapes into the pinch chamber througin noles around the gun electrode. The quontity of gas 


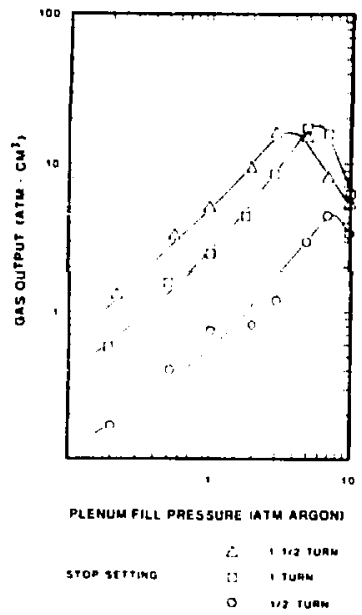

Figure A.3. Totol gos valve output as a function of plenum pressure for vorious stop sefings.

which posses the cothode plane is thus dependent on the pressure differences befween three chambers. Therefore, consider o simplified model of this geometry as shown in tig. A.4. The quantities $P_{0}, P_{1}$. and $P_{2}$ are the pressures, as o function of time, in the plenum, the intermediate volume, and the pinch chomber, respectively. The rolumes $V_{0}$ and $V_{1}$ ore $13.3 \mathrm{~cm}$ and $.72 \mathrm{~cm}$. The areo $A_{f}$ is $.20 \mathrm{~cm}^{2}$. The area Ao $(t)$ varies with time os 


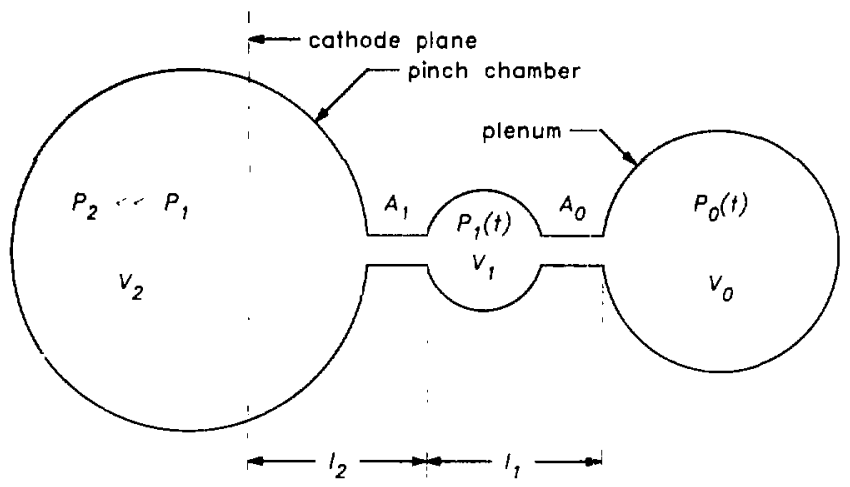

Figure A.4. Model of pulsed gas valve and pinch chamber used in gas flow calculations.

$$
\begin{aligned}
A_{0}(t) & =0, t \cdot t_{0} \\
& =\frac{A_{\max }}{w}\left(t-t_{d}\right), t_{d} \cdot t \cdot t_{d}+w \\
& =\frac{A_{\max }}{w}\left(t_{d}+2 w-t\right), t_{d} \cdot t<2 w+t_{d} . \\
& =0,+>2 w
\end{aligned}
$$

The quantify $t_{d}$ is the deloy time in the gas sclve opening, and $w$ is fime for the valve disk to reoch the stop. It is a function of the volve stop position, being the number of turns of the stop multiplied by a constant and divided by the disk velocity. With a $12 \mathrm{kV}$ copasitor 
geometrical factor equal to $.0907 \mathrm{~cm} / t u r n$. A $A_{\max }$ is also a function of the stop position, and is the number of turns multiplied by a constant, $.274 \mathrm{~cm}^{2} / \mathrm{turn}$.

The gas quantities in each chamber, as a function of time, are found by considering sonic flow befween the chambers. The sonic flow assumption is allowed if the pressure drop befween successive chambers is more than a factor of two. ${ }^{1}$ Only for the largest valve openings, and for only very short durations is this condition not met. For an ideal gas, the sound velocity is $\sqrt{\gamma k T / M}$ and thus varies only with the temperature. The flow through a restricted orifice is a Joule-Thompson process, which conserves enthalpy, and hence requires, with the case of an ideal gas, that the temperature on either side of the orifice be the same. ${ }^{2}$ Thus, it is sufficient to consider the sound velocity to be a constant. For argon, at room temperature, it is $3.19 \times 10^{4} \mathrm{~cm} / \mathrm{sec}$.

The rate of change in the quantity of gos in the plenum is proportional to the difference in chamber pressures. (The pressure drop due to turbulence in the orifice is insignificont because of the finite dimensions, and is therefore neglected.)

$$
\frac{d}{d t}\left(P_{0} v_{0}\right)=-v_{s} A_{0}\left(P_{0}-P_{1}\right)
$$

The rote of change of the gas quantity in the intermediate chamber is proportional to the gas flow in minus the gas flow out. Initially, 
sound tronsit time $l, / v_{s}$ ofter the flow starts on the right end. Further, the time dependence of the pressure at the left end must also be displaced by the transit time. For convenience, define $f_{1}=1, / v_{s}$, the transit time across the intermediate volume, and $t_{2}=t_{2} / v_{s}$, the transit time from the chamber opening to the cathode plane.

$$
\begin{aligned}
\frac{d}{d t}\left(P_{1} v_{1}\right) & =v_{s} A_{0}\left(P_{0}-P_{1}\right), t<t, \\
& =v_{s} A_{0}\left(P_{0}-P_{1}\right)-v_{s} A_{1} P_{1}\left(t-p_{1}\right), t \geq 1,
\end{aligned}
$$

Since the chamber volume is so much larger than the plenum, its pressure rise is very small, and the effect on the flow rate is negligible. The time rate of change of the quantity $Q$ of gas entering the chamber $\left(Q=P_{2} V_{2}\right)$ is also displaced by the transit time. At the opening to the chamber it is

$$
\begin{aligned}
\frac{d Q}{d t} & =0, t<t, \\
& =v_{s} A_{1} P_{1}\left(t-t_{1}\right), t \geq t_{1} .
\end{aligned}
$$

Integrate eq. A.4 to obtain the total quantity which enters the chamber. 


$$
\begin{aligned}
Q(t) & =\int_{t,}^{t} v_{s} A_{1} P_{1}\left(\tau-t_{1}\right) d \tau \\
& =\int_{0}^{t-t_{1}} v_{s} A_{1} P_{1}(\tau) d \tau, t \geq t_{1}
\end{aligned}
$$

The quantity of gas that has passed the cathode plane at time $t$ is the value of eq. A.5 displaced by the transit time $t_{2}$.

$$
Q(t)=v_{s} A_{1} \int_{0}^{t-\left(t_{1}+t_{2}\right)} P_{1}(\tau) d \tau, t \geq t_{1}+t_{2}
$$

The solution thus requires the knowledge of the time behavicr of $P,(t)$, which must be obtained from eqs. A.2 and A.3. These equations were solved numerically with a $4^{\text {th }}$ order Runge-Kutta scheme ${ }^{3}$ with the initial conditians that $P_{0}(0)$ is a given constant, and that $P_{1}(0)$ and $Q(t)$ are zero. The results for several stop settings are shown in fig. A.5. Fig. 2.5 shows the same results, expanded with a semi-logarithmic scale, for the total quantity of gas $Q(t)$.

The numerical solver wos compared with on analytical solution for the case that $A_{0}$ is constant, and $t_{d}, t_{1}$, and $t_{2}$ are zero. The two 


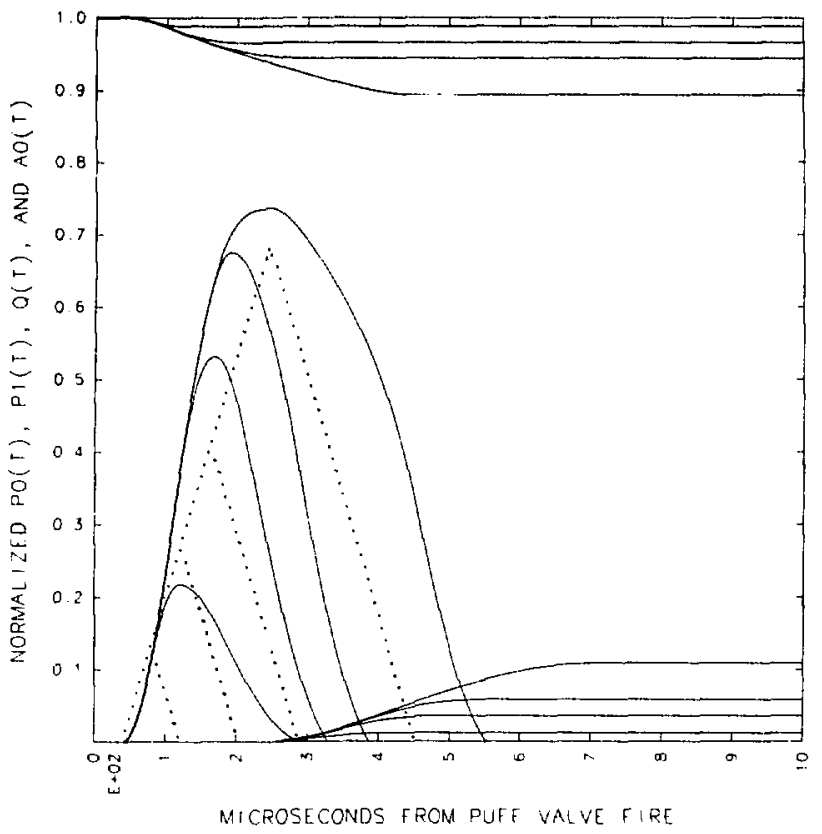

Figure A.5. The numerical solution of $P_{0}(t)$ and $P_{1}(t)$ normalized to the initial plenum pressure $P_{0}(0)$, and $Q(t)$, normalized to the initial quantity in the plenum for $1 / 2,1,1,1 / 2$, and $21 / 2$ turns of the valve stop. The family of curves in the upper portion of the frame is $P_{0}(t)$. Those lines in the lower right-hand corner are $Q(t)$. The remaining family of solid lines is $P_{i}(t)$. The dotted lines are $A_{o}(t)$, the plenum opening areas, in units of $\mathrm{cm}^{2}$. 
sufficient for this model.

It is worth noting. thot for times ofter about $500 \mu s e c$, the quantity of gas in the chamber does not increase appreciably. This indicates that measurements of gas output, os shown in fig. A.3, would also give good estimates for the quantity in the chamber at this time. Comparing fig. A.5 with fig. A.3 at 1 Atm shows a disagreement by almost a factor of ten. however. As stated on page 159, the data displayed in fig. A.3 is from an earlier version with a different coil construction. It was replaced with a newer version because its primary coil was shorting out as it was pulsed. This would hove led to a smaller driving force, and hence smaller disk velocity, which would have left the valve open longer. For that valve it is therefore not valid to consider the total output equivalent to the gas present at the time the pinch bank is fired. Rather, it is necessary to consider the same problem, but with a lower disk velocity. Fig. A.6 shows the results of the same analysis, but with a disk velocity arbitrarily set to one-fourth of that used for generating fig. A.5. From this plot it can clearly be seen that the quantity of gas in the chamber at $500 \mu s e c$ is approximately equivalent for the two valves. However, the asymptotes are very different, showing that the total gas output measurement does not provide sufficient informotion to know the quontity in the chamber at the bank fire time. The reason the total quantities differ with the two coses is that one valve is open longer thon the other. 


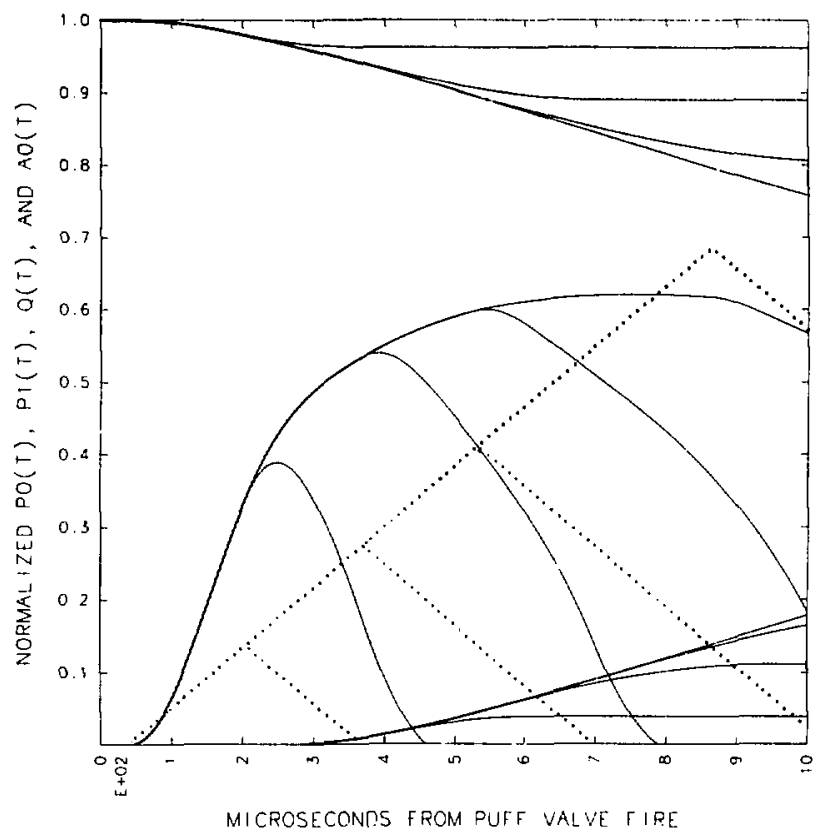

Figure A.6. The numerical solution of $P_{0}(t), P_{1}(t)$, and $Q(t)$ for the case that the disk velocity is one-fourth that used for fig. A.5. Refer to fig. A.5 for a discription of the families of curves.

The output of the Berkeley puffatron valve can also be modeled with this technique by adiusting the various orlfice areas and plenum volumes. The steel rod which makes the seal is connected to the 
spring, and driven off by pulsing the solenoidal coil. The motion of the rod was not measured, but it can be estimated by considering the magnetic flux of the coil supplying on impulse over a period of $\sim 100 \mu s e c$. Doing this, the rod velocity is estimated to be $3 \times 10^{3} \mathrm{~cm} / \mathrm{sec}$. The restoring force of the spring is considered to have a much smaller effect on the rad velocity. Thus, for the tine scalo of the experiment, the valve is considered to remain open. With these estimates, and the measured geometry of the valve, the gas sutput as a function of time is calculated as shown in fig. A.7. As is seen in the figure, the motion of the rod after $600 \mu \mathrm{sec}$ would not have an appreciable effect since most of the gas has left the plenum prior to this time.

References for appendix A

1J. K. Vennord, Elementary Fluid Mechanics, $4^{\text {th }}$ ed. (Wiley, New York, 1963), PP. 156-158.

${ }^{2} \mathrm{~F}$. Reif. Fundamentals of Statistical and Thermal Physics, (McGraw-Hill. New York, 1965), p. 180.

3Handbook of Mathematical Functions. edited by M. Abramowitz and I. A. Stegun, (Dover, New York, 1968), P. 897, sec. 25.5.18. 


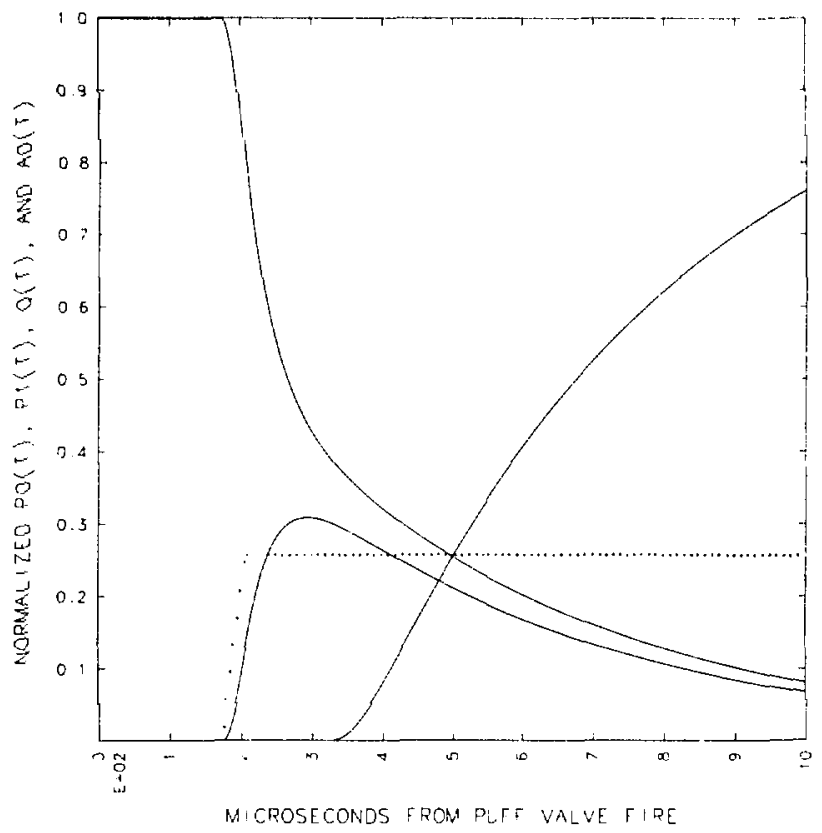

Figure A.7. The numerical solution for normalized values of $P_{0}(t)$, $P_{1}(t)$, and $Q(t)$ for the Berkeley puffatron. Refer to fig. A.5 for a description of the curves. 


\section{APPENDIX $B$}

\section{Laser Interferometry and Abel Inversion}

B.1. General consideratians

Use of an interferometer provides on aesthetically pleosing approach to making electron density measurements of a plasmo. The probing radiation, be it ultraviolet, visible, infra-red, or microwave, need not be of sufficient strength to affect the plasma. The technique also does not require that the resulis depend on a calibrotion since the number of fringe shifts depends only on the frequency of the probing radiation, and size and density of the plasma. The techniques of microwave and laser inierferametry are discussed by a number of authors.1-3 Jahoda 4 has given a number techniques for spatially resolved laser interferometry and holographic interferometry. 
The number of fringe shifts $N(y)$ in a Mach-Zehnder interferometer is the difference between the path lengths of the two legs of the interferometer, divided by the wavelength $\lambda$. The path lerigth in each leg is adjusted such that there is no fringe shift withow the plasma. When the plasma is present, the index of refraction $\zeta$ of the experimental leg differs from that of the reference leg, and results in a fringe shift.

$$
N(y)=\int_{0}^{l(y)}(1-\zeta(x, y)) \frac{d x}{\lambda}
$$

The index of refraction of a plasma, for frequencies much greater than the electron cyclotron frequency (which it is by several orders of magnitude for the lasers used), is

$$
\zeta=\left(1-\frac{\omega_{p}^{2}}{\omega^{2}}\right)^{1 / 2}
$$

The electron plasma frequency $\omega_{p}$ is a function of the electron density $n_{0}$ and mass $m_{\theta}$, and the electron charge $e$. In mks units

$$
\omega_{p}=\left(\frac{n_{e} e^{2}}{E_{0} m_{e}}\right)^{1 / 2} .
$$

For $\omega \gg \omega_{p}$, expand eq. (B.2) in a binomial series, keeping only the 
first term, and substitute into eq. (B.1).

$$
N(y)=A(\lambda) \int_{0}^{L(y)} n_{0}(x, y) d x
$$

where $A(\lambda)$ conloins all the constants, and in mks units equols

$$
A=\frac{\mu_{0} e^{2}}{8 \pi^{2}} \lambda .
$$

With cgs units eq. (B.4) is identical to eq. (2.1) of chopter 2.

Volues of $\mathrm{A}$ are given for $\mathrm{He}-\mathrm{Ne}$, argon-ion, and $\mathrm{CO}_{2}$ losers in Table B.I. Table B.I shows the validity of the smoll $\omega_{p} / \omega$ opproximation for a density of $10^{18} / \mathrm{cm}^{3}$. A similor porameter, the electron cut-off density, is also shown. For electron densities above this value the index of refraction is imaginary, and the laser beam is rellecied.

\section{B.2. Abel Inversion}

The geometry of the experiment wos such that it was necessary to look across the pinch, rather than to look down the axis. If it can bo ossumed thot the plasmo consists of a number of concentric shells, each 
Toble B.1. Comparison of parameters for argor-ion. He-Ne, and $\mathrm{CO}_{2}$ losers. The quantity $\omega_{p} / \omega$ is calculated for an electron density of $10^{18} / \mathrm{cm}^{3}$. The parameter $A$ is defined in eq. (B.4).

\begin{tabular}{lcccc}
\hline $\begin{array}{c}\text { Laser } \\
(\mathrm{m})\end{array}$ & $\begin{array}{c}\text { Cut-off densily } \\
\left.\text { (electrons } / \mathrm{cm}^{3}\right)\end{array}$ & $\begin{array}{c}\omega_{p} / \mathrm{L} \\
\left\langle\mathrm{cm}^{2}\right\rangle\end{array}$ \\
\hline Argon-ion & 0.5145 & $4.2 \times 10^{2 !}$ & .002 & $2.31 \times 10^{-18}$ \\
He-Ne & 0.6328 & $2.8 \times 10^{21}$ & .003 & $2.84 \times 10^{-18}$ \\
$\mathrm{CO}_{2}$ & 10.6 & $9.9 \times 10^{18}$ & .05 & $4.75 \times 10^{-17}$
\end{tabular}

the fringe shift measured at several positions perpendicular to the axis. This technique is called on Abel inversion and is discussed by several outhors. 5.6

The inversion can be easily derived. Consider the geomeiry of fig. B.1 witin a collimated laser beam propagating in the x-direction. Consider $m$ concentric shells of outer radius $r_{i}$, and $m$ measured frequency shifts of positions $y_{k}$ olong the $y$-axis, where the indices $i$ and $k$ range from 1 to $m$. For the outer sheil, the fringe shift is o result of the density in the $k=m$ shell only. For beam poths intersecting more shells, the fringe shift is the sum of contributions of several shells. Since the measured fringe shift is an average over an increment $\Delta y$, calculale the average contribution from each shell 


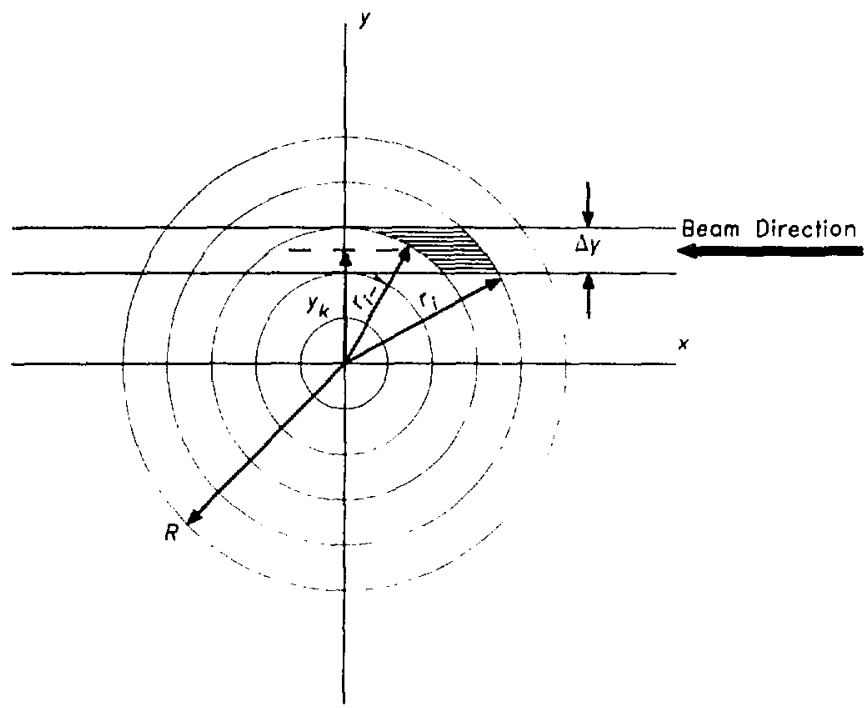

Figure B.1. Geometry used in the Abel inversion. The plosmo consists of $m$ concentric cylindrical shells of uniform density $n\left(r_{i}\right)$.

segment within the increment. The contribution from the cross-hatched segment in $\mathrm{fig} . \mathrm{B} \cdot 1$ is

$$
N_{i}\left(y_{k}\right)=\frac{A}{\Delta y} \int_{y_{k}-\Delta y / 2}^{y_{k}+\Delta y / 2} d y \int_{\sqrt{r_{i}^{2}-1-y^{2}}}^{\sqrt{r_{i}^{2}-y^{2}}} n\left(r_{i}\right) d x .
$$

The contributions from all the segments in the beam path are summed to obtain the total fringe shift at $y_{k}$. Since the sum is symmetrical about the $y$-axis, only the contributions of the $1^{\text {st }}$ quadrant are used, 


$$
\begin{aligned}
N\left(y_{k}\right) & =2 \sum_{i=k}^{m} N_{i}\left(y_{k}\right) \\
& =2 \frac{A}{\Delta y} \sum_{i=k}^{m} \int_{y_{k}-\Delta y / 2}^{y_{k}+\Delta y / 2} d y \int_{1, r_{i-1-y^{2}}}^{r^{2}-y^{2}} n\left(r_{i}\right) d x .
\end{aligned}
$$

Note olso, that because of symmetry, $N\left(-y_{k}\right)=N\left(y_{k}\right)$. Hance, only positive values of $y_{k}$ need be considered.

The integral can be simplified by noting that the electron density is constant on each shell, thus ollowing it to be factored out of the integral. The integrations over $x$ and $y$ can then be performed. Doing first the $x$ integrotion, and defining o geometrical factor $a_{i k}$. it is found that

$$
\begin{aligned}
N\left(y_{k}\right) & =\frac{\Delta}{\Delta y} \sum_{i=k}^{m} n\left(r_{i}\right) \int_{y_{k}-\Delta y / 2}^{y_{k}+\Delta y / 2} d y\left(i r_{i}^{2}-y^{2}-i r_{i-1}^{2}-y^{2}\right) \\
& =\sum_{i=k}^{m} a_{i k} n\left(r_{i}\right) .
\end{aligned}
$$

where $a_{i k}$ is defined as, 


$$
o_{i k}=2 \frac{A}{\Delta y} \int_{y_{k}-\Delta y / 2}^{y_{k}+\Delta y / 2} d y\left(\sqrt{r_{i}^{2}-y^{2}}-\sqrt{r_{i}^{2}-y^{2}}\right), i \neq k \text {. }
$$

For the case $i=k$, the second term in the porentheses in eqs.

and ( 8.9 ) is zero since the $y$-axis is the lower limit of integration for the integral over $x$.

Note that the limits of the cemaining integral $y_{k}+3 y / 2$ and $y_{k}-\Delta y / 2$, ore equal to the shell rodil $r_{k}$ and $r_{k-1}$, respectively. Integrating for $i=k$. it is found that

$$
\begin{aligned}
a_{k k}= & \frac{A}{\Delta y}\left(-r_{k-1}\right) r_{k}^{2}-r_{k-1}^{2} \\
& \left.+\frac{\pi}{2} r_{k}^{2}-r_{k}^{2} \sin ^{-1} \frac{r_{k-1}}{r_{k}}\right)
\end{aligned}
$$

For $i>k$, the coefficient is

$$
\begin{aligned}
a_{i k}= & \frac{A}{\Delta y}\left[r_{i}\left(\sqrt{r_{i}^{2}-r_{k}^{2}}-\sqrt{r_{i-1}^{2}-r_{k}^{2}}\right)\right. \\
& -r_{k-1}\left(\sqrt{r_{i}^{2}-r_{k-1}^{2}}-\sqrt{r_{i-1}^{2}-r_{k-1}^{2}}\right) \\
& +r_{i}^{2}\left(\sin ^{-1} \frac{r_{k}}{r_{i}}-s_{i n^{-1}} \frac{r_{k-1}}{r_{i}}\right) \\
& \left.+r_{i-1}^{2}\left(\sin ^{-1} \frac{r_{k-1}}{r_{i-1}}-\sin ^{-1} \frac{r_{k}}{r_{i-1}}\right)\right], i>k .
\end{aligned}
$$


The solution to this set of equations is readily seen upon writing out the sum of eq. (B.8).

$$
\begin{gathered}
N\left(y_{m}\right)=a_{m m} n\left(r_{m}\right) \\
N\left(y_{m-1}\right)=a_{m-1, m-1} n\left(r_{m-1}\right)+a_{m-1, m} n\left(r_{m}\right) \\
\cdot \\
\cdot \\
N\left(y_{1}\right)=u_{1,1} n\left(r_{1}\right)+a_{1,2} n\left(r_{2}\right)+\ldots+a_{1, m} n\left(r_{m}\right)
\end{gathered}
$$

Since the geometrical factors $a_{i k}$ are known, it is a simple process of back-substitution to determine the shell densities, given the fringe shift as a function af position. That is, the density of the $i=m$ shell is determined by sclving the first line of eq. (B.12). This value is substituted into the second line, which is solved for the $m^{-1}$ shell density, which is then substituter into the next equation. This process continues until all the $n_{\theta}\left(r_{i}\right)$ are determined.

The electron line density $\mathcal{N}$ of the pinch is also easily determined with the approach. It is the integral of the electron densitv over the cross-sectional area of the pinch.

$$
\mathcal{N}=\int d x \int d y n_{\theta}(x, y)
$$

The limits of integration are such that the entire pinch cross-section 
is included in the integral. This integrol, however, is identical to the sum and integral of eq. (B.7) for on oreo of thickness $\Delta y$. Therefore, sum the contributions from each $\Delta y$, and substitute in $\mathcal{N}$ for the integrul.

$$
\begin{aligned}
2 \sum_{k=1}^{m} N\left(y_{k}\right) & =4 \frac{A}{\Delta y} \sum_{k=1}^{m} \sum_{i=k}^{m} \int_{y_{k}-\Delta y / 2}^{y_{k}+\Delta y / 2} d y \int_{\sqrt{r_{i}^{2}-r^{2}}}^{\sqrt{r_{i}^{2}-y^{2}}} n\left(r_{i}\right) d x \\
& =\frac{A}{\Delta y} \mathcal{N}
\end{aligned}
$$

The factor of two appears becouse the sum over $k$ only includes the positive values of $y$. Equation (B.14) is solved for the line density $\mathcal{N}$.

$$
\mathcal{N}=2 \frac{\Delta y}{A} \sum_{k=1}^{m} N\left(y_{k}\right)
$$

An algorithm was written to compute the shell densities and the line density. The resulting points were fit to a 5 th or lower order polynomial which was constrained to hove a zero slope on the axis. Test cases were run to verif, that it worked correctly, and to see how coneitive the dansity mansuraments nre to varintinns in nranisinn af 
the fringe shift measurements. The results of several runs of varying

Table 8.2. Density measurement errors fram the Abel inversion routine. Shell densities and line densitios determined by the inversion routine are compared with the input far a uniform density test case. The standard deviation of the density for each shell, and the errar in the line density is shown to vary with the precision of the fringe shift used, and with the number of shells.

\begin{tabular}{|c|c|c|c|c|c|c|}
\hline $\begin{array}{c}\text { significont } \\
\text { figures }\end{array}$ & $\begin{array}{l}\% \text { sto } \\
\text { in sh }\end{array}$ & $\begin{array}{l}\text { idard devi } \\
11 \text { densit }\end{array}$ & $\begin{array}{l}\text { ition } \\
\text { with }\end{array}$ & $\%$ error & $\begin{array}{c}\text { in line } \\
\text { with }\end{array}$ & nsity \\
\hline in & 3 & 5 & 10 & 3 & 5 & 10 \\
\hline input & she IIs & shel Is & shells & shells & she $\mid$ Is & shel Is \\
\hline 4 & .02 & .03 & .03 & .01 & $<.01$ & $<.01$ \\
\hline 3 & .07 & .17 & .29 & .02 & .03 & $<.01$ \\
\hline 2 & 1.49 & 2.84 & 2.30 & .12 & .12 & .03 \\
\hline 1 & 6.12 & 17.8 & 27.9 & 5.36 & 4.36 & .12 \\
\hline
\end{tabular}

precision are given in table B.2. The dota shows that increasing the number of shelis increoses the standaro deviation in the shell densities, but olso decruses the error in the line density. Therefore, one needs to decide which measurement is most important in choosing the number of shells for the Abel inversion. 


\section{References for appendix $B$}

${ }^{9} M$. A. Heald, and C. B. Wharton, Plasma Diagnostics with Microwaves, (Wiley. New York, 1965).

${ }^{2}$. A. Alpher, and D. R. White, in Plasma Diagnostic Techniques, edited by R. H. Huddlestone, and S. L. Leanard, (Academic, New York, 1965), pp. $431-476$.

${ }^{3}$ F. C. Jahoda, G. A. jawyer, in Methods of Experimental Physics, Vol. 9, Part B, edited by H. Griem (Acodemic, New :ork, 1971).

${ }^{4} \mathrm{~F}$. C.Jahoda, and R. E. Siemor, "Holographic Interferometry Cookbook", Las Alamos Scien, Ific Laboratory Report LA-5058-MS, 1972.

5W. Lochte-Holtgreven, Plosmo Diagnostien, (North-Holland, Amsterdam, 1968), pp. 184-186. 


\section{APPENDIX C}

\section{Determination of the Effective Ion Charge}

In using the electron density meosurements to defermine the Bennett temperature, it is necessary to first determine the average charge per ion $Z_{\text {ove }}$. The technique used to find $Z_{\text {ave }}$ depends on which model is used for the ionization, radiation, recombination, and collision processes. For a plasma in thermodynamic equilibrium, i.e., one that behaves as a black-body radiator, the Saho-Eggert equations ore used.1,2 Nost laboratory plasmas are not black-body radiators in that they do not reabsorb most of the emitted radiation, and they are thus termed notically thin plasmas. If collision processes still play a dominant role, the plasma can be in local thermodynamic equilibrium (LTE). Thus the populations of the bound electron states of an ion or atom can obey a Bnltzmann distribution sespite the radiation losses. For this class of plasmas the Saha-Eggert equations are also valid, provided the electron density is suffiuiently high. MeWhirter ${ }^{3}$ gives a 
criterion for LTE which places a lower bound on the electron density.

$$
n_{0} \geq 1.7 \times 10^{14} T_{0}^{1 / 2} \phi(p, q)^{3} \mathrm{~cm}^{-3}
$$

$T_{\text {a }}$ is the electron temperature, and $\phi(p, q)$ is the excitation potential of level p from level $q$. Both are in units of $e V$.

The maximum value of $\Phi$ is the potential from the ground state to the firstexcited state, for a given ionization stage. As higher temperatures are considered, the atom is more highly ionized, and the polential to the first excited state much larger. Therefore, despite the only mild explicit dependence on the temperature, eq. (C.1) is strongly dependent on the electron temperature because of he cubic dependence on $\Phi$, which is a strong function of $T_{e}$. An argon plasma, for example, with $T_{e} \geq 100 \mathrm{eV}$. must have an electron density graater than $10^{21} \mathrm{~cm}^{-3}$ to be in LTE. Thus, at higher temperatures, and with lower electron densities, it is necessary to consider other models to determine the effective chorge.

With lower densities the ionization levels become less dependent on the electron density, but strongly dependent on various rodiative processes. One model which considers some of these processes is the corona model, which is discussed in detail by Mathirter. 4 House ${ }^{5}$ has used this model to calculate the ionization levels of the elements $H$ through $\mathrm{Fe}$, for temperotures ranging from $\log _{10} T(\mathrm{eV})=-0.5$ to 3.0 . 
to be populated. His results, for lawer temperotures, are genaraliy valid for $n_{0} \leq 10^{15} \mathrm{~cm}^{-3}$.

Thus, it is obvious, that a model which would correctly predict the effective charge as a function of temperature must include LTE effects for low temperatures, and corona like effects at higher temperatures. This has been done by Hyman ${ }^{6}$ using a technique proposed by Raizer ${ }^{7}$ to obtain on approximate solution to the Soha equations. Since this method is used to obtain figs. C.1 - C.6, I will explain his opprooch.

The standard corona-like equations expressing the degree of ionization are

$$
\frac{n_{z+1}}{n_{z}}=\frac{S\left(T_{e}\right)}{\alpha\left(T_{\theta} \cdot n_{\theta}\right)} .
$$

The quantities $n_{z}$ and $n_{z+1}$ are the traction of ions in the $z$ and $z+1$ charge states. respectively. $S\left(T_{e}\right)$ is the normal collisional ionization coefficient, and $\alpha\left(T_{e}, n_{e}\right)$ is the recombination coefficient, which can be written as the sum of radiative and three-body collisional terms.

$$
\alpha\left(T_{\theta}, n_{\theta}\right)=a^{r}\left(T_{\theta}\right)+n_{\theta} \beta\left(T_{\theta}\right)
$$

The superscript $r$ designates the radiative term, and $\beta$ is the collisional term. For plasmos in LïE, $n_{\theta} \beta \gg \alpha^{r}$, and eq. (C.2) becomes 
the Saha equation. In the opposite limit, it becomes the corono equation.

Eq. (c.2) can be eosily solved, following the method of Raizer.? by considering $n_{z}$ and $x_{z}$, the ionization potential of the $z^{\text {th }}$ ian, to be continuous functions of the charge $z$. Therefore.

$$
n(z+1)=n(z)+\frac{d n}{d z} \quad(\Delta z=1)
$$

Substituting eq. (C.4) into eq. (C.2) gives

$$
1+\frac{1}{n} \frac{d n}{d z}=\frac{5}{\alpha} .
$$

For a given electron temperature and density, only a relatively few ionization stafes ore populated, and $n(z)$ is a sharply peakea function. Therefore, look for a solution of eq. (C.5) with $d n / d z=0$. Doing this, eq. (C.5) becomes

$$
s\left(T_{\theta}, \bar{x}\right)=\alpha\left(T_{e}, n_{\theta}, \bar{x}\right)
$$

where $\bar{x}$ is an effective ionization potential at the peak. Generally, $\bar{x}$ will not equol the ionizotion potentiai of cny of the ionization states, buf rather will lie between the values of two consecutive stotes. The effective charge is then determined by using a table of 
corresponding to $\bar{x}$. However, the interpolated value (call it $z^{*}$ ) is not quite the average charge. Rather, as is seen by eqs. (C.6) and (C.2), it is the volue for which $n_{z}+1=n_{z} \cdot$. The average charge is thus the interpolated value plus $1 / 2, z_{\text {ove }}=z^{*}+1 / 2$.

Specific expressions for $a$ and $S$ are taken from McWhirter. Thus, eq. (C.7) reduces to the transcendental equation

$$
e^{\bar{x} / T} \cdot\left(a \bar{x}^{11 / 4}+\frac{b n_{0}}{T_{0}^{3 / 4}}\right)=T_{0}^{3 / 4} .
$$

where $a$ and $b$ are constants equal to $7.82 \times 10^{-9}$ and $1.65 \times 10^{-22}$, respectively. The units of $\bar{x}$ and $T_{e}$ ore in ev, and $n_{0}$ is in $\mathrm{cm}^{-3}$.

Eq. (C.7) is solved for $\bar{\chi}$ by an interval-halving technique, to produce a plot of $\bar{\chi}$ vs. $T_{e}$, as is shown in fig. C.l for an electron density $1.0 \times 10^{21} \mathrm{~cm}^{-3}$. This solution agrees with fig. 1 of Hyman's paper. More sophisticated root finders, which use the gradient of the function, do not work. The gradients away from the solution are so large that the methods do not converge to the solution in a reasonable number of iterations.

The average ion charge $Z_{a v g}\left(T_{0}\right)$ is determined by interpolation using the ionization potentials given by Lotz. 8 Plots of $Z_{\text {ave }} v$. $T$. for electron densities $10^{16}-10^{20} \mathrm{~cm}^{-3}$ are shown in figs. C.2 - C. 6 . The values are found to agree to within $10 \%$ with the calculations of House $^{5}$ at high temperatures, and. for lower temperatures, to within $10 \%$ 


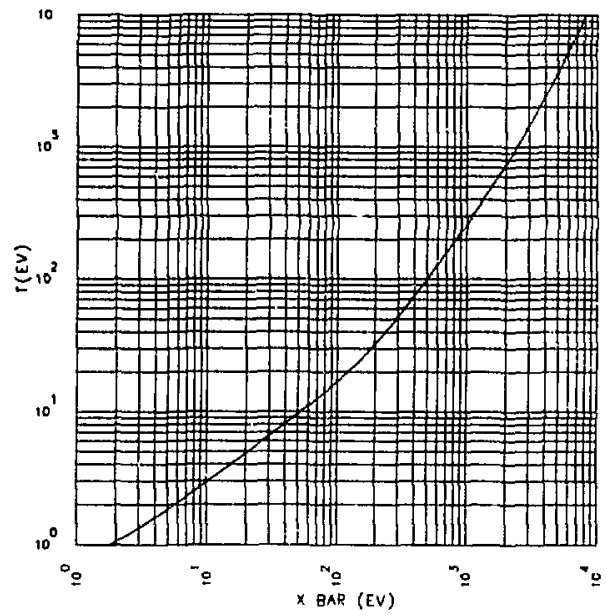

Figure C.1. Solution to eq. (C.7). Plot of $T$. versus $\bar{x}$ with $n_{.}=1 \times 10^{21} \mathrm{~cm}^{-3}$.

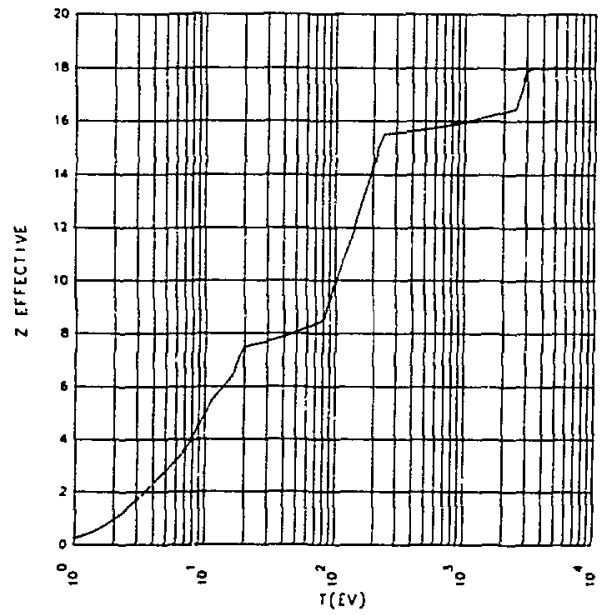




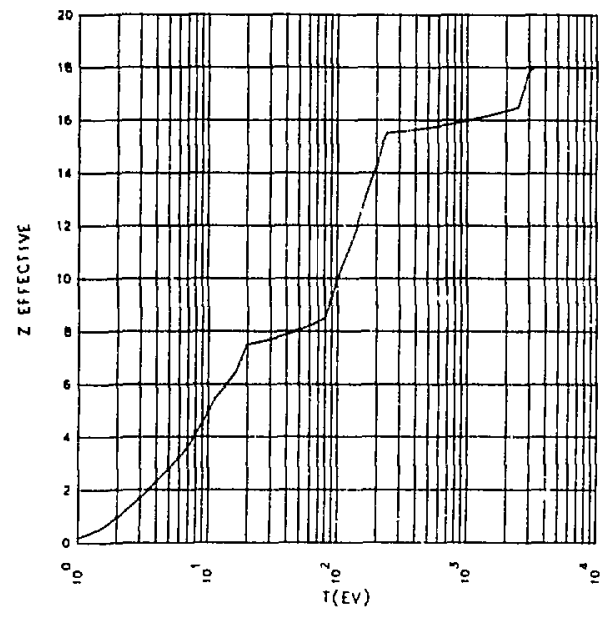

Figure C.3. Plot of $Z_{\text {ave }}$ vs. $T_{\text {. }}$ for argon wi ih $n_{0}=1 \times 10^{17} \mathrm{~cm}^{-3}$.

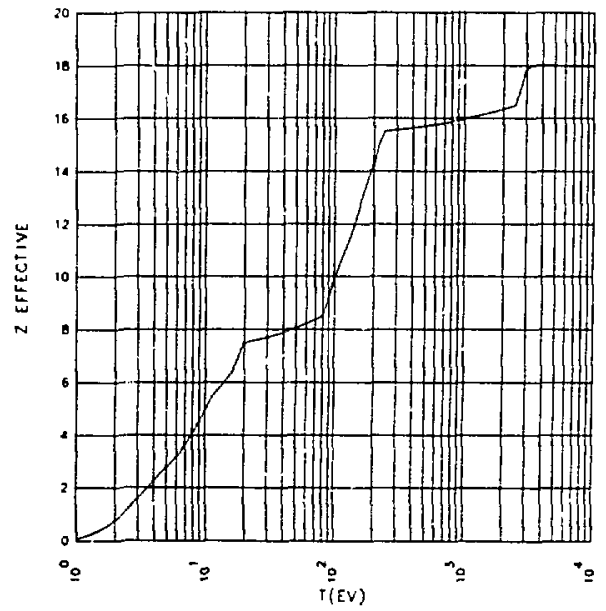




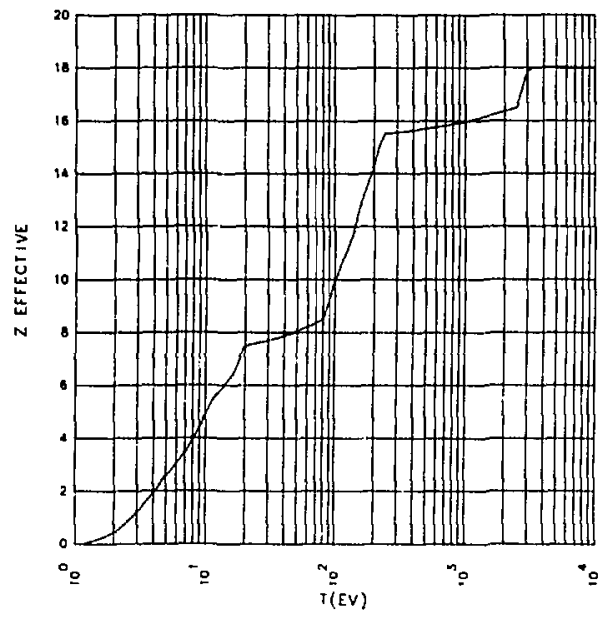

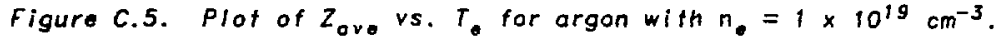

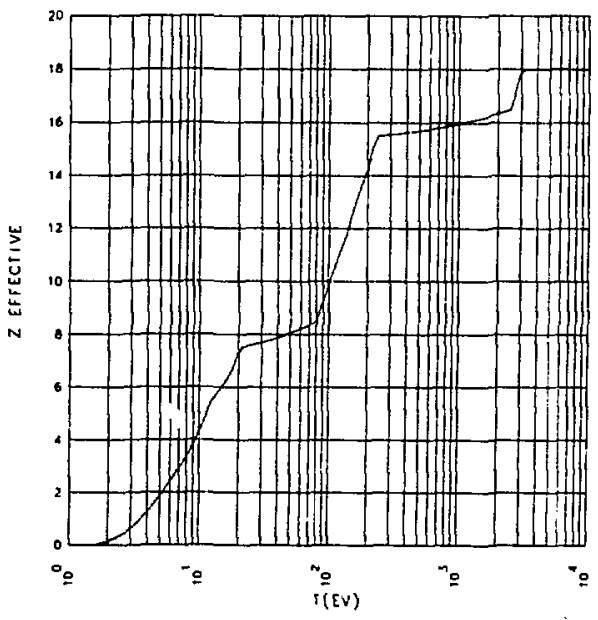


References for appendix $C$

'J. Richter, in Plosma Diagnostics, edited by W. Lochte-Hollgreven (North-Halland, Amsterdam, 1968), pp. 15-20.

${ }^{2} R$. W. P. MoWhirter, in Plasma Diagnostic Tochniques, edited by R. H. Huddlestone, and S. L. Leonard (Academic, Now Yark, 1965), chap. 5.

${ }^{3}$ MaWhirter, ibid., p. 206.

MaWhirter, ibid., pp. 208-222.

5L. L. House, Astrophy. J. Suppl. Ser. 18, 307 (1964).

${ }^{6}$ H. A. Hyman, Appl. Phys. Lett. 25, 553 (1974).

7Y. B. Zel'dovich and Y. P. Raizer, Physics of Shock Waves and High-Temperature Hydrodynamic Phenomena, Vol. 1 (Academic, New York. 1966), pp. 201-207.

sw. Lolz. Institut Fur Plasmaphysik Report No. IPP 1/49, 1966; W. Lotz, J. Opt. Soc. Am. 57, 873 (1967).

${ }^{9}$ S. S. Chowdhury, J. Quont. Spectrosc. Radiat. Transfer 9, 129 (1969). 


\section{APPENDIX D}

\section{Electron Density Profiles}

In calculating growth rates with the diffuse current, ideal MHD theory, it is necessary to know the pressure and current profiles. If the pinch is considered to have a uniform temperafure across its radius, then the pressure is linearly proportional to the density. The density profiles are determined, in furn, from Abel inverting the laser interferograms. In this appendix, several example interferograms of shots with roughly the same paramelers as those of figures 3.8 and 3.9 ore given. The Abel inverted densities are connected by a least squares fit to (1) a quadratic profile, $n=n_{0}\left[1-(r / a)^{2}\right]$; and (2) to a Bennett profile, $n=n_{0} /\left[1+(r / a)^{2}\right]^{2}$. These loast squares fits differ from those in the rest of the paper in that they are parameterized by only two constants. (The other abel inversions are generally fit to a power series with more than two terms.) As is obvious from the following figures, the Bennett profile provides a much 
is a gaussian profile, $n=n_{0} \exp \left[-(r / a)^{2}\right]$. It generally fif the density points better than the quadratic, but not as well as the Bennett profiles. 

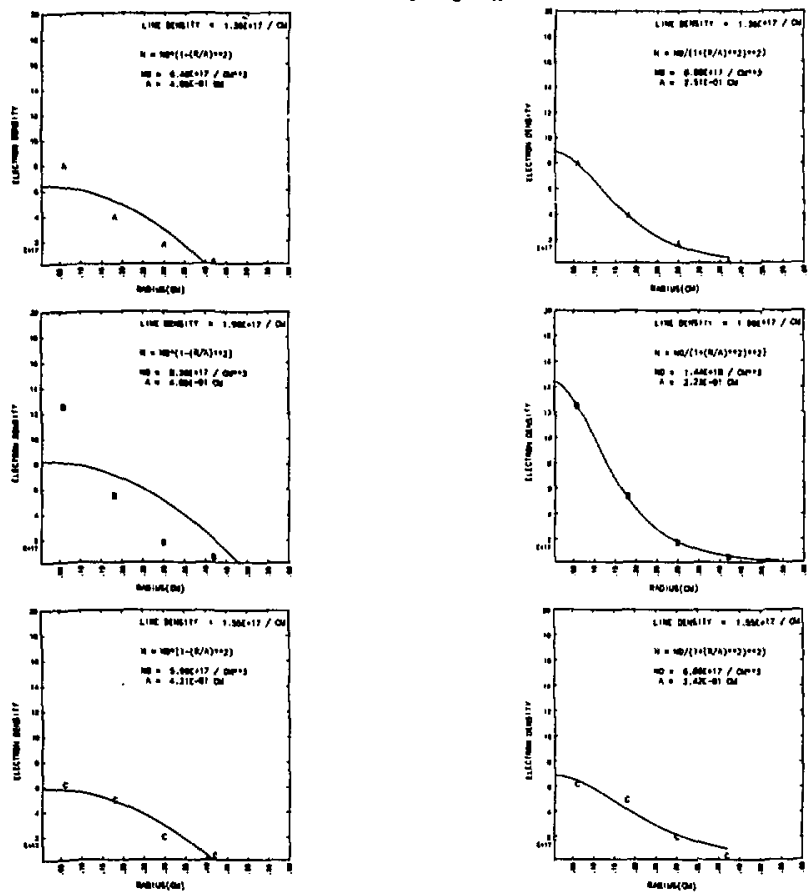

$$
n=n_{0}\left[1-(r / a)^{2}\right]
$$$$
n=n_{0} /\left[1+(r / a)^{2}\right]^{2}
$$

Flgure 0.1. Electron density profiles of shot 1113, comparing least squares fifs of the Abel inverted densities to quadratic and Bennett profiles. 

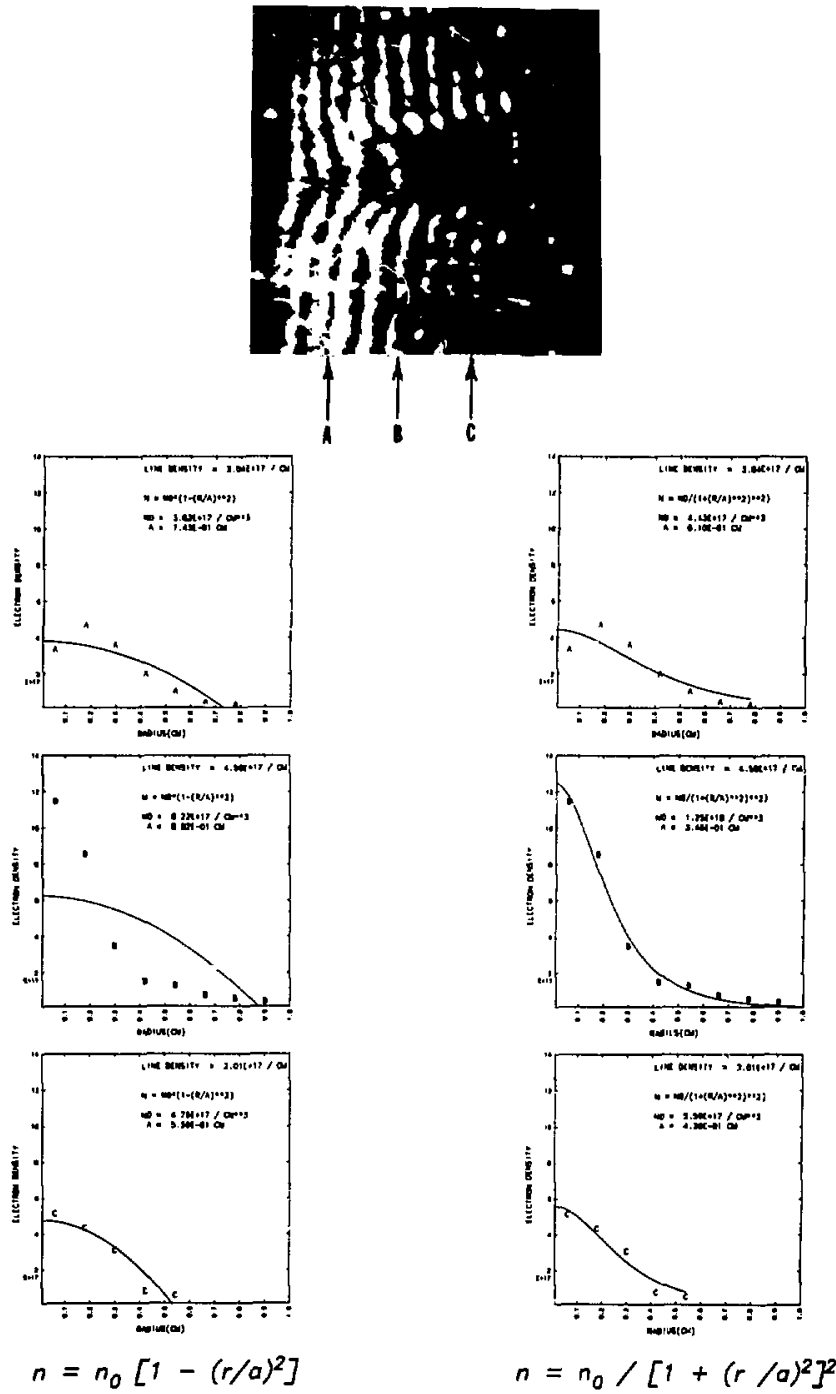

Figure D.2. Electron density profiles of shot 1115, comparing least squares fits of the Abel inverted densitios to quadratic and Bennett profiles. 


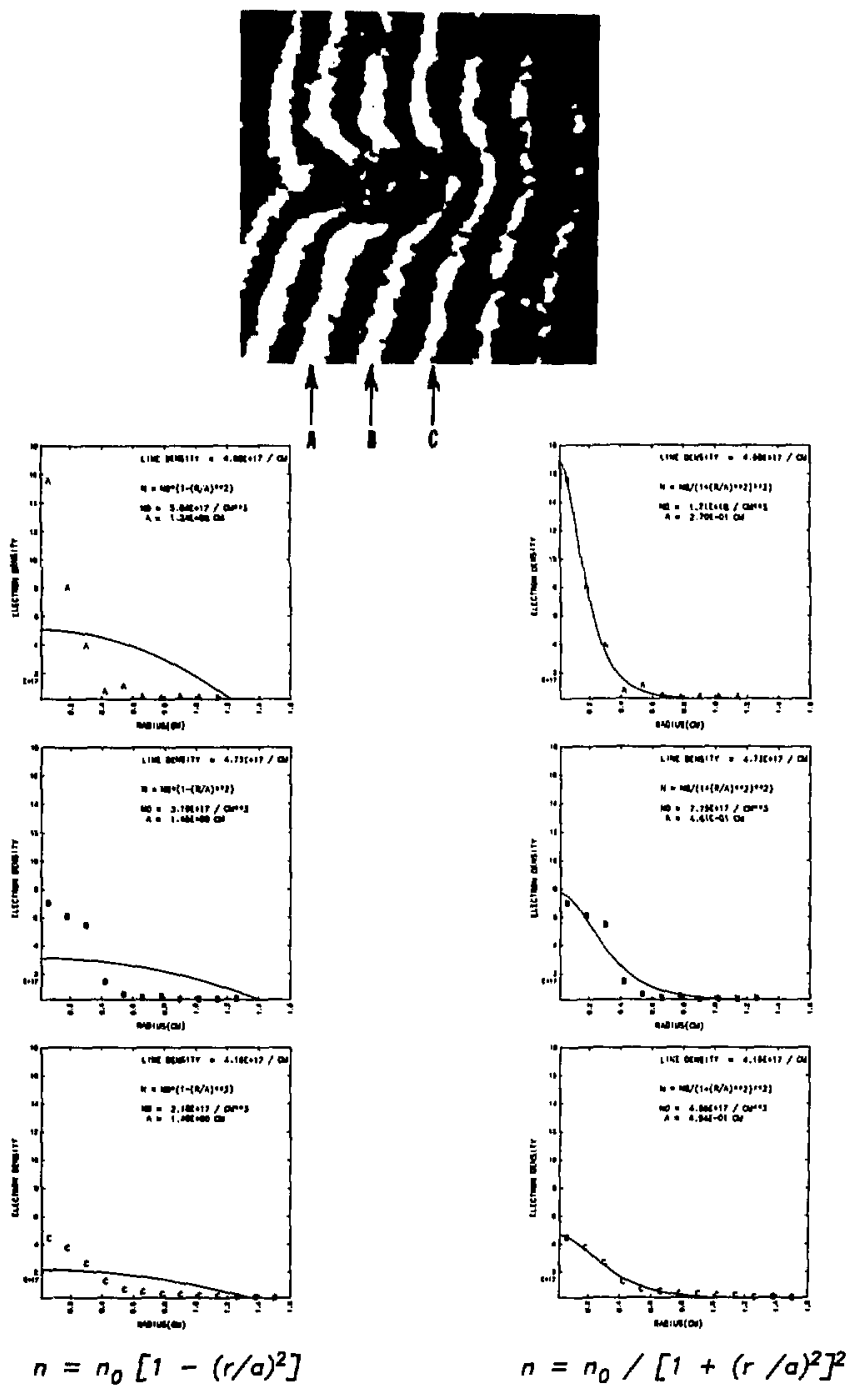

Flgure D.3. Eloctron densliy proflles of shot 2115, comporing least squares fits 

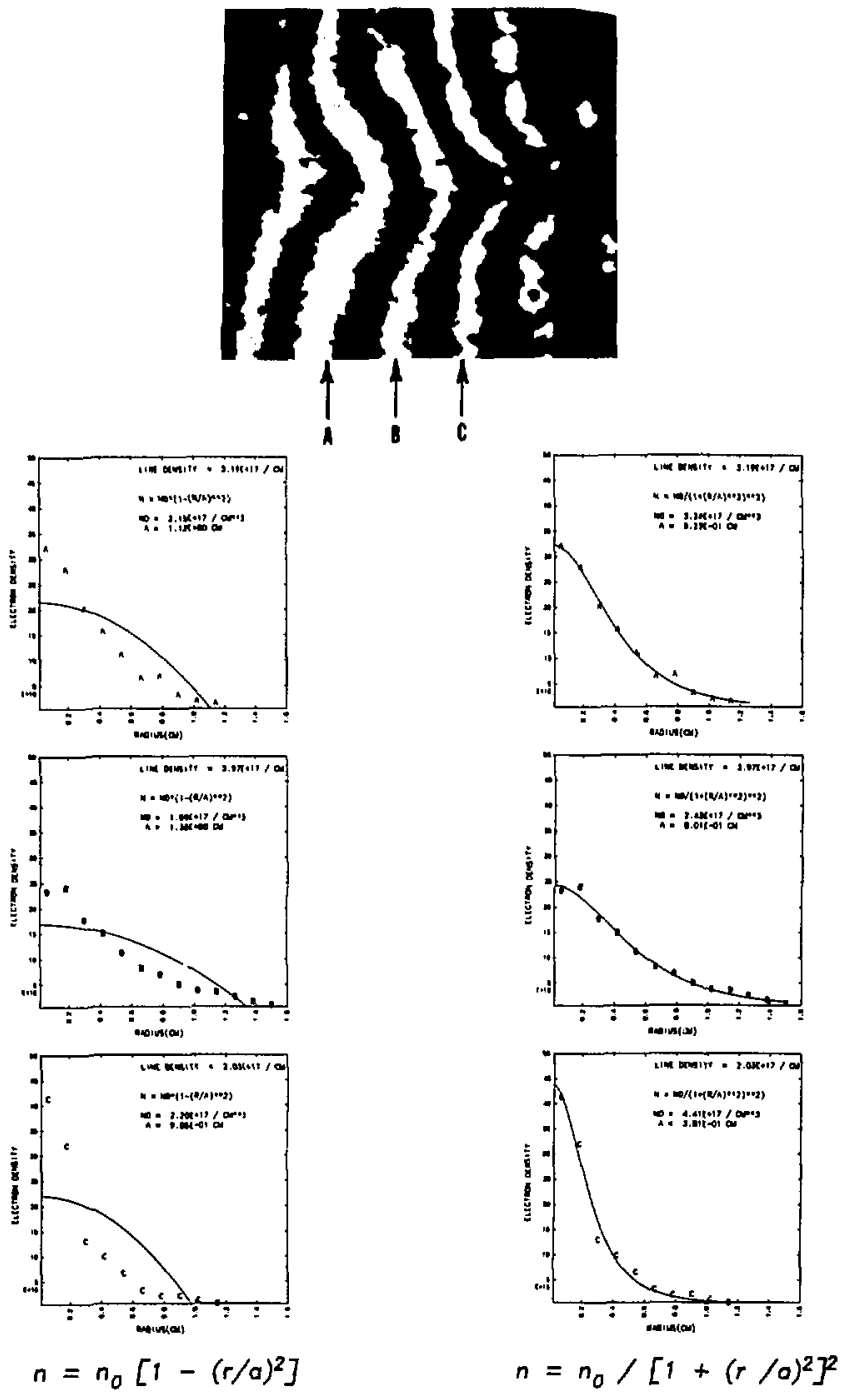

Figure D.4. Electron density profiles of shot 3115, comporing least squares fits of the Abol inverted densities to quadratic and Bennott profiles. 


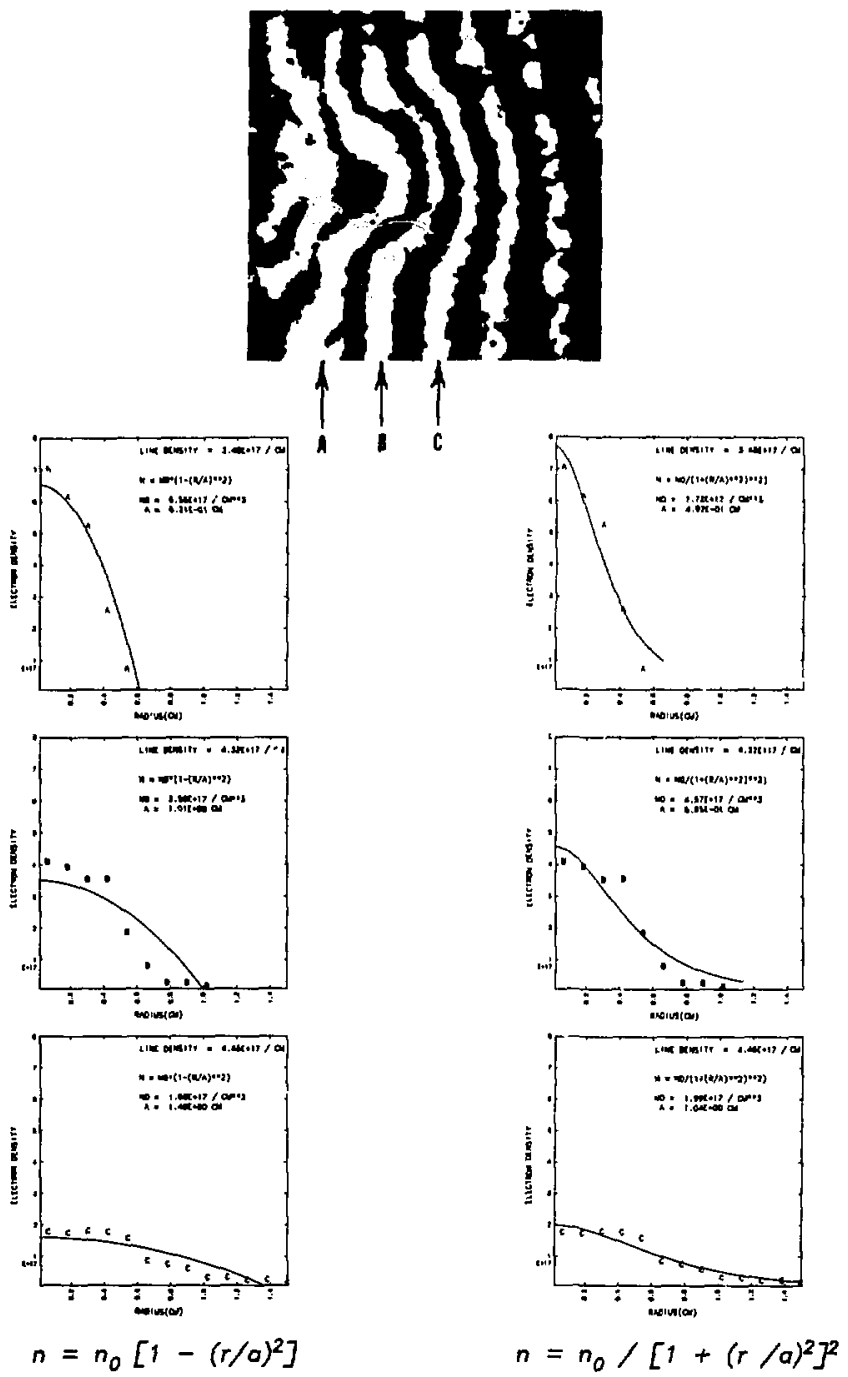

Figure 0.5. Electron density profiles of shof 6115, comparing least squores fits of the Abel inverted densities to quadratic and Bennetf profiles. 